\title{
Functional cortical cartography
}

Citation for published version (APA):

Frost, M. A. (2012). Functional cortical cartography. [Doctoral Thesis, Maastricht University]. Maastricht University. https://doi.org/10.26481/dis.20120914mf

Document status and date:

Published: 01/01/2012

DOI:

10.26481/dis.20120914mf

Document Version:

Publisher's PDF, also known as Version of record

\section{Please check the document version of this publication:}

- A submitted manuscript is the version of the article upon submission and before peer-review. There can be important differences between the submitted version and the official published version of record.

People interested in the research are advised to contact the author for the final version of the publication, or visit the DOI to the publisher's website.

- The final author version and the galley proof are versions of the publication after peer review.

- The final published version features the final layout of the paper including the volume, issue and page numbers.

Link to publication

\footnotetext{
General rights rights.

- You may freely distribute the URL identifying the publication in the public portal. please follow below link for the End User Agreement:

www.umlib.nl/taverne-license

Take down policy

If you believe that this document breaches copyright please contact us at:

repository@maastrichtuniversity.nl

providing details and we will investigate your claim.
}

Copyright and moral rights for the publications made accessible in the public portal are retained by the authors and/or other copyright owners and it is a condition of accessing publications that users recognise and abide by the legal requirements associated with these

- Users may download and print one copy of any publication from the public portal for the purpose of private study or research.

- You may not further distribute the material or use it for any profit-making activity or commercial gain

If the publication is distributed under the terms of Article $25 \mathrm{fa}$ of the Dutch Copyright Act, indicated by the "Taverne" license above, 


\title{
FUNCTIONAL CORTICAL CARTOGRAPHY
}

\author{
Martin A. Frost
}


No part of this publication may be reproduced, stored in an automated data system or transmitted in any form by any means, electronic, mechanical or photocopying, recording, or otherwise without the prior written permission of the author.

(C) Martin A. Frost, Maastricht 2012

ISBN 978-94-6203-152-4 


\section{Functional Cortical Cartography}

Ter verkrijging van de graad van doctor aan de Universiteit Maastricht, op gezag van de Rector Magnificus, Prof. Dr. L. Soete volgens het besluit van het College van Decanen, in het openbaar te verdedigen op vrijdag 14 september 2012 om 14:00 uur door

Martin A. Frost

geboren op 25 maart 1982 te Sydney, Australië 


\section{Promotor:}

Prof. Dr. Rainer Goebel

\section{Copromotor:}

Prof. Dr. Elia Formisano

\section{Beoordelingscommissie:}

Prof. Dr. Peter de Weerd (voorzitter)

Prof. Dr. John Ashburner (University College London)

Dr. Kamil Uludag

Dr. Alard Roebroeck 


\section{CONTENTS}

Chapter 1 Introduction

Chapter 2 Measuring Structural - Functional Correspondence:

Spatial variability of Specialised Brain Regions After

Macro-Anatomical Alignment

Chapter 3 Improved Correspondence of Resting State Networks after Macro-Anatomical Alignment

Chapter 4 One or Two Analyses? An Integrated Approach for Whole-Cortex Macro-Anatomical and ROI-Based Functional Alignment

Chapter 5 An Investigation Into the Structural and Functional Development of Human Superior Temporal Cortex

$\underline{\text { Summary }}$

Samenvatting

$\underline{\text { Acknowledgements }}$

Curriculum Vitae 
CHAPTER 1

INTRODUCTION 


\section{Introduction}

Since pre-history maps have been created to record and display humankind's knowledge of the world they observed around them. Even in the early days of our cave dwelling ancestors, humans felt drawn to depict their knowledge of spatial relations of their world around them in a cartographical composition (Smith, 1982). Throughout the centuries and across cultures, maps have proven to be the most effective way to record and display discoveries as they were being made. Whether it was navigational maps which allowed trade to flourish between people of distant lands, or the mapping of useful resources buried in the ground beneath us, from mapping the galaxies in our quest to solve the mysteries of the universe or the mapping of the human genome to understand the building blocks of all life. The creation of accurate maps has been and will continue to be at the forefront of the most important human endeavours.

An interesting similarity between mapping the world and mapping the brain can be found in the fact that at the centre of both endeavours lays a system of cardinal directions that allow one to orient themselves. Whereas geographic maps use the magnetic points of North, South, East and West, maps of the brain can be navigated using anatomical orientations, superior/inferior, medial/lateral, ventral/dorsal etc. As well as the need for orientation, both brain and geographical mapping requires a correction for geometric distortions. In order to display the spherical earth on a 2 dimensional sheet, early cartographers devised mathematical solutions to ensure their charts allowed sailors to safely navigate the dangerous stretches of ocean. In the same way, recent advances in cortical cartography has given rise to similar considerations, of how best to approach the representation of the brain; as a three dimensional volume, as a cortical surface, as a flat map, or as a sphere?

On closer inspection early cartography and early brain mapping, share some further remarkable similarities. Both have, in the past, been guilty of not only depicting reality in their maps but have suffered due to an inability to observe, and importantly, accurately measure that which is to be mapped (see figure 1, a map of the Far East from the an early, $16^{\text {th }}$ Century atlas, by Abraham Ortelius). As a result distant places were often not mapped accurately. Early attempts to create maps of functional brain areas were as flawed as the maps created by some of the pioneering cartographers. The pseudo- 
science of compartmentalising anatomical areas of the brain (and indeed the cranium in general) and assigning personality and other mental traits and functions to these areas, known as phrenology, is the most well known example of early attempts of brain mapping where proper observation and measurement were not employed as part of the map making process. Just as some maritime map making included the edge of the earth, or "true" indications of sites of mermaid habitation, phrenology was based on un-founded claims about the relationship between brain function and structure.
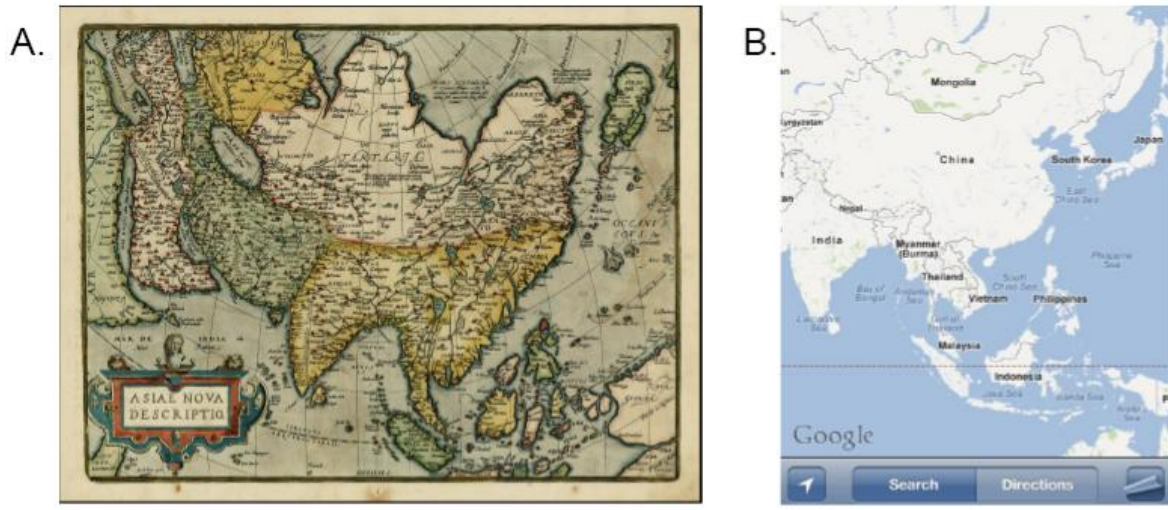

Figure 1. (A) Map of Asia Abraham Ortelius "Asiae Nova Descriptio" as mapped in the 16th Century Source : raremaps.com and (B) Map of the same region in Asia as mapped in the $21^{\text {st }}$ Century source Google maps on Apple iPhone. While the $16^{\text {th }}$ century map is a fine depiction of Asia, and without doubt is based on tireless efforts of measurement, it is only with technological advances such as satellite imagery and GPS that allow for the precise maps we so easily access today.

Brain mapping made large strides forward with the work of Paul Broca, who, after careful study of lesion patients, was able to localise the seat of language production to near the "third frontal convolution of the left side of the brain" (Obituary in Science, 1880). This was quickly followed by the observation of Carl Wernicke that a lesion to the left posterior, superior temporal gyrus affect one's ability to comprehend language. Despite the fact that these two discoveries were compartmentalising the human brain, in the same way as the phrenologists did, the difference lies in the fact that their claims were made after careful observation and measurement of behaviour and the brain itself, albeit post-mortem.

Indeed the $19^{\text {th }}$ century saw many great leaps in human brain mapping. Besides Broca and Wernicke's work there was the anatomical maps of Kor- 
binian Brodmann (Brodmann, 1909). Brodmann labelled 48 distinct brain areas based on micro-anatomical "cytoarchitectonics" with each border distinguishing between areas composed of a different arrangement of cells. Never before had such a rigorous and thorough investigation been carried out into the organisational structure of the human brain. In fact, that his maps are still widely consulted today in neuro-imaging is testament to his outstanding efforts, especially seeing that a paper only a few years old can usually be considered obsolete in this field.

A.

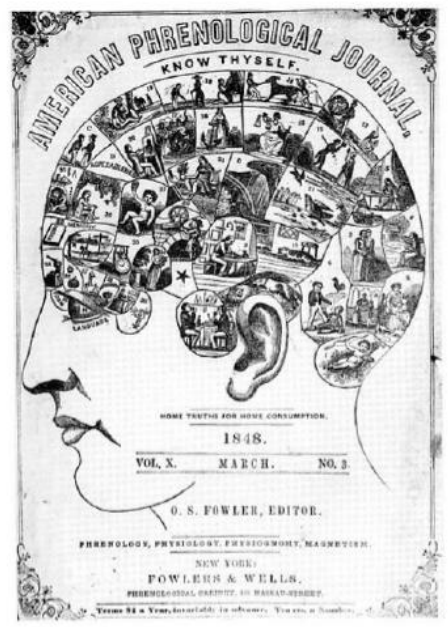

B.

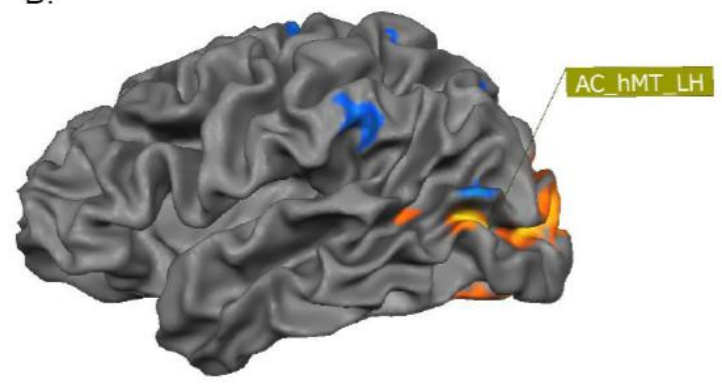

Figure 2. The brain mapping equivalents of figure 1 above. (A) A 19th Century idea of brain mapping from the American Phrenological Journal from 1848, source Wikipedia and (B) a map of the brain as measured by using modern fMRI technology. Again accurate measurement tools available today allow the structure and function of the living human brain to be mapped with astounding accuracy.

While detailed anatomical post-mortem investigations of the brain were possible by Brodmann and others, studying the brain in terms of function required the development of more advanced tools than the microscope and scalpel such as Position Emission Tomography (PET) and more recently functional magnetic resonance imaging (fMRI) exploiting the BOLD (Blood oxygenation level dependent) effect. The fMRI BOLD signal arises from a biological phenomenon whereby an oversupply of oxygenated blood is transported to the site of active neurons; this alters the magnetic properties in the region of this activated area, giving rise to a signal measured using the fMRI scanner. The development of fMRI has brought forth the world of modern brain mapping which bears some resemblance to previous attempts 
in that certain brain areas are labelled as being involved in a particular sensory, motor or cognitive process. However just as satellite photography and GPS have made mapping and navigating the earth a more accurate endeavour, so too has fMRI made true functional brain mapping possible. In both geographical mapping as well as brain mapping, the introduction of tools capable of precise observation and measurement are responsible for the advancement in both these fields. Without the ability to accurately measure, whether it be the coastline of Norway or the activation of the primary visual cortex, maps, although objects of beauty and fascination, are not useful tools. It is only through accurate measurement that maps reach their true potential.

This thesis describes some of the latest analysis tools used to create accurate maps of the functioning human brain. With an understanding and appreciation of the importance of mapping, here we present our work on functional cortical cartography. 


\section{Background of normalisation and alignment}

Despite the fact that thousands of experiments have been carried out where functional brain areas are examined and mapped, there has been, to a certain extent a lack of attention paid to how these functionally defined regions correspond with underlying anatomy. The field has long acknowledged the link between the brain's structure and function, indeed Brodmann himself noted that "parts of organs which differ structurally must have different functions" (Franz, 1912) however the importance of this relationship is often overlooked in functional neuroimaging. The problem as Devlin and Poldrack (2007) noted, is that cognitive neuroscience is a field which involves experts from many fields and as a consequence, one cannot assume that all who work in the field are experts in neuro-anatomy. For this reason, procedures used to relate functional information to structure are done, "based largely on informal advice that varies considerably from lab to lab" (Devlin and Poldrack, 2007). Simple and workable solutions are utilised that, although they provide some ability to localise functional areas in relation to structure, they lack the precision required for a proper understanding of the relationship between cortical structural and functional. By neglecting to thoroughly solve issues relating structure to function, studies can suffer from a loss of statistical power as a proper spatial correspondence is not established. In a way it is equivalent to overlaying a map of a road system on a satellite image of the same area, yet shifted by a few meters, or drawn on a slightly different scale. More appropriate for brain mapping is the comparison that one map would be moved, rotated or stretched in a different way at different locations with respect to the other map reflecting non-linear distortions. Consequently a proper one-to-one relationship between the two maps is not established and problems ensue. In the same way group fMRI studies must bring data from individuals to a common space to establish proper spatial correspondence between individuals. In the field of human brain mapping this is done via normalisation procedures.

The most widely used normalisation procedures automatically morph an individual subject to fit a template brain. The two most commonly used volumetric normalisation schemes are the Talairach space, based on the classic stereotactic atlas created by Talairach and Tournoux from one postmortem examination (Talairach and Tournoux, 1980) and the MNI template space (Evans et al., 1993; Evans et al., 1992). Both the Talairach co-ordinate 
space and the MNI template system have been extremely important tools to create spatial correspondences between subjects for group analysis. As well as provide a system which allows not only the precise reporting of a locus of activation in $\mathrm{x}, \mathrm{y}, \mathrm{z}$ Cartesian co-ordinates, but also allow for the collating of data across vast amounts of studies for the purpose of meta-analysis.

Despite these benefits of Talairach and MNI registration these normalisation procedures have severe drawbacks. The major problem being that the same coordinate can refer to different anatomical areas across subjects. It has often been shown that even the most distinct macro-anatomical landmarks in the brain are often not brought into alignment after this normalisation procedure. This of course has detrimental effects on the power of group statistics as the activation on which the statistics are carried out can potentially come from a different macro-anatomical location in each subject.

In order to overcome these issues, solutions have been sought by not looking at the brain in volume space, but as a cortical surface. In fact the cortex can be considered a sheet of neuronal tissue, of just a few millimetres thickness. Over the course of the evolution of the human brain it has become increasing convoluted, in order to fit the larger surface area of cortex into the rather limited space of the cranial cavity. Pioneering work from a number of labs demonstrated the visualisation and analytic breakthrough of cortical surface reconstructions (Dale et al., 1999; DeYoe et al., 1996; Fischl et al., 1999a; Goebel et al., 1998; Sereno et al., 1995; Thompson and Toga, 1996; Van Essen, 2005; Wandell et al., 2000). The creation of flat maps and inflated hemispheres from surface reconstructions of the cortical sheet meant that the hidden sulcal regions were made clearly visible. This made it possible to display activations from disparate cortical regions on single, canonical, representation of the cortex instead of a series of slices. Finally the use of surface based representations reflected the intrinsic topology of the cortex allowing the calculation of geodesic distances between brain regions as opposed to Euclidian distances in volume space

The shift to 2D surface representations of fMRI data also has allowed a leap forward in alignment procedures to increase the power of group analysis by increasing anatomical correspondence across subjects. Fischl et al. showed the potential of the spherical co-ordinate system in the creation of group averaged surface maps (Fischl et al., 1999b) as opposed to a system where alignment is carried out after manual selection of macro-anatomical land- 
marks. The spherical co-ordinate averaging method demonstrated much improved anatomical alignment and provided first evidence of improved concomitant alignment of functional regions. The use of cortex based alignment (CBA) schemes has continued to grow and has been extended by transforming functional time course data directly in cortex space allowing one to carry out multi-subject analyses such as general linear model (GLM) analysis and Independent Component Analysis (ICA) directly in cortex space (Formisano et al., 2004; Goebel et al., 2006). 


\section{Outline of thesis}

The first empirical study presented in this thesis (chapter two) investigates how strongly functional areas correspond with macro-anatomical landmarks. This was done by minimising macro-anatomical variability across subjects through an advanced cortical alignment procedure. A battery of functional localising experiments was used to localise functional areas across the whole cortex in a group of subjects. By comparing the difference in the degree to which these functional regions overlap across subjects before and after alignment we could measure the degree to which these functional regions respect macro-anatomical landmarks. The rationale being that if there is a high degree of overlap across subjects in a functional area after anatomical variability has been minimised, this functional area is strongly bound to macro-anatomy. We observed that the degree to which functional regions "respected" anatomy differed across the cortex with some functional regions such as the frontal eye fields (active during eye movements), were strongly bound to anatomical regions, whereas other areas, such as the fusiform face area (active during the presentation of images of faces) was not brought into alignment to the same extent, therefore not as tightly bound to macro- anatomy.

In the next chapter (chapter three) we continued the investigation of the relationship between cortical anatomy and function, as measured by fMRI, however we turn our attention away from discrete functional areas (covering just a few square centimetres of cortical surface) and focus on distributed functional networks which span the whole cortex. The same experimental logic was followed in this experiment, namely, resting state network maps were obtained in a group of subjects and anatomical variability was minimised using the same surface based alignment scheme as in the previous study and differences in spatial similarity of these functional networks were compared before and after anatomical alignment. Similar results were obtained in that, having maximised correspondence of anatomical regions across subjects we observed an increase in the correspondence in these functional networks as well. Furthermore, as with discrete functional regions, the degree to which functional networks respect macro-anatomy varied, highlighting the fact there is not a single structural functional correspondence value. 
The fourth chapter was inspired by the findings of the precious two studies. We had realised that, while using a surface based, anatomically driven alignment scheme, substantial statistical gains can be made in brain mapping studies as the greater correspondence of anatomy results in a concomitant correspondence of functional regions. Alignment based purely on macroanatomy, however, is not capable of perfectly aligning all functional regions. For this reason we developed a new alignment procedure which incorporates anatomical and functional information from each subject. Having carried out an alignment in this way we were able to create a group cortical reconstruction which is well aligned in terms of both anatomy and function. Due to the anatomical variability across subjects, many brain mapping experiments choose to focus their analysis on one (or a few) particular region of interest so as to circumvent the problem of sub-optimal statistics resulting from the lack of anatomical correspondence in standard whole-brain normalisation. This means that two analyses need to be carried out, one on the region of interest alone, and a separate 'whole brain' analysis. Using this alignment scheme which integrates anatomical and functional information, we propose that two analyses may not be necessary. After an integrated structural and functional alignment one is able to carry out a whole brain analysis in the confidence that little (if any) statistical power is lost due to spatial variation in the location of functional regions of interest.

The fifth and final chapter of the thesis utilises many of the analysis approaches developed in the previous studies, and applies them to the human auditory system, examining the development of anatomical and functional areas related to auditory processing from childhood, though adolescence and into adulthood. We incorporated measures of anatomical variability, by labelling anatomical landmarks and measuring the inter-subject distance, with measures of variability in location of functional regions to observe the changes in this part of the brain. We observed that the brain continues to develop past childhood and that certain features of the auditory system are not fully established until adulthood. 


\section{References}

Brodmann, K. (1909). Vergleichende Lokalisationslehre der Grosshirnrinde. Barth Verlag, Leipzig.

Dale, A.M., Fischl, B., Sereno, M.I. (1999). Cortical surface-based analysis. I. Segmentation and surface reconstruction. Neuroimage 9, 179-194.

Devlin, J.T., Poldrack, R.A. (2007). In praise of tedious anatomy. Neuroimage 37, 1033-1041; discussion 1050-1038.

DeYoe, E.A., Carman, G.J., Bandettini, P., Glickman, S., Wieser, J., Cox, R., Miller, D., Neitz, J. (1996). Mapping striate and extrastriate visual areas in human cerebral cortex. Proc Natl Acad Sci U S A 93, 2382-2386.

Evans, A.C., Collins, D.L., Mills, S.R., Brown, E.D., Kelly, R.L., Peters, T.M. (1993). 3D statistical neuroanatomical models from 305 MRI volumes. Nuclear Science Cymposium and Medical Imaging Conference, 1993., 1993 IEEE Conference Record, San Francisco, CA, USA, pp. 1813-1817.

Evans, A.C., Marrett, S., Neelin, P., Collins, L., Worsley, K., Dai, W., Milot, S., Meyer, E., Bub, D. (1992). Anatomical mapping of functional activation in stereotactic coordinate space. Neuroimage 1, 43-53.

Fischl, B., Sereno, M.I., Dale, A.M. (1999a). Cortical surface-based analysis. II: Inflation, flattening, and a surface-based coordinate system. Neuroimage 9, 195-207.

Fischl, B., Sereno, M.I., Tootell, R.B., Dale, A.M. (1999b). High-resolution intersubject averaging and a coordinate system for the cortical surface. Hum Brain Mapp 8, 272-284.

Formisano, E., Esposito, F., Di Salle, F., Goebel, R. (2004). Cortex-based independent component analysis of fMRI time series. Magn Reson Imaging 22, 1493-1504. 
Franz, S.I. (1912). New Phrenology. Science 35, 321-328.

Goebel, R., Esposito, F., Formisano, E. (2006). Analysis of functional image analysis contest (FIAC) data with BrainVoyager QX: From single-subject to cortically aligned group general linear model analysis and self-organizing group independent component analysis. Hum Brain Mapp 27, 392-401.

Goebel, R., Khorram-Sefat, D., Muckli, L., Hacker, H., Singer, W. (1998). The constructive nature of vision: direct evidence from functional magnetic resonance imaging studies of apparent motion and motion imagery. Eur $J$ Neurosci 10, 1563-1573.

Leonard, C.M., Puranik, C., Kuldau, J.M., Lombardino, L.J. (1998). Normal variation in the frequency and location of human auditory cortex landmarks. Heschl's gyrus: where is it? Cereb Cortex 8, 397-406.

Pantazis, D., Joshi, A., Jiang, J., Shattuck, D.W., Bernstein, L.E., Damasio, H., Leahy, R.M. (2010). Comparison of landmark-based and automatic methods for cortical surface registration. Neuroimage 49, 2479-2493.

Sereno, M.I., Dale, A.M., Reppas, J.B., Kwong, K.K., Belliveau, J.W., Brady, T.J., Rosen, B.R., Tootell, R.B. (1995). Borders of multiple visual areas in humans revealed by functional magnetic resonance imaging. Science $268,889-893$.

Smith, C.A. (1982). The emergence of 'maps' in European rock art: A prehistoric preoccupation with place. Imago Mundi: The International Journal for the History of Cartography 34, 9-25.

Talairach, J., Tournoux, P. (1980). Co-planar sterotaxic atlas of the human brain. Thieme, Stuttgart.

Thompson, P., Toga, A.W. (1996). A surface-based technique for warping three-dimensional images of the brain. IEEE Trans Med Imaging 15, 402417. 
Van Essen, D.C. (2005). A Population-Average, Landmark- and Surfacebased (PALS) atlas of human cerebral cortex. Neuroimage 28, 635-662.

Wandell, B.A., Chial, S., Backus, B.T. (2000). Visualization and measurement of the cortical surface. J Cogn Neurosci 12, 739-752.

Abraham Ortelius Map of Asia. Barry Lawrence Ruderman Antique Maps. Retrieved March 25th, (2012), from.www.raremaps.com 


\section{MEASURING STRUCTURAL - FUNCTIONAL CORRESPONDENCE: SPATIAL VARIABILITY OF SPECIALISED BRAIN REGIONS AFTER MACRO- ANATOMICAL ALIGNMENT}

Corresponding publication:

Frost, M.A. \& Goebel, R. (2012). Measuring structural-functional correspondence: Spatial variability of specialised brain regions after macro-anatomical alignment. Neuroimage 59, 1369-1381. 


\begin{abstract}
The central question of the relationship between structure and function in the human brain is still not well understood. In order to investigate this fundamental relationship we create functional probabilistic maps from a large set of mapping experiments and compare the location of functionally localized regions across subjects using different whole-brain alignment schemes. To avoid the major problems associated with meta-analysis approaches, all subjects are scanned using the same paradigms, the same scanner and the same analysis pipeline. We show that an advanced, curvature driven cortex based alignment (CBA) scheme largely removes macro-anatomical variability across subjects. Remaining variability in the observed spatial location of functional regions, thus, reflects the "true" functional variability, i.e. the quantified variability is a good estimator of the underlying structuralfunctional correspondence. After localising 13 widely studied functional areas, we found a large variability in the degree to which functional areas respect macro-anatomical boundaries across the cortex. Some areas, such as the frontal eye fields (FEF) are strongly bound to a macro-anatomical location. Fusiform face area (FFA) on the other hand, varies in it's location along the length of the fusiform gyrus even though the gyri themselves are well aligned across subjects. Language areas were found to vary greatly across subjects while a high degree of overlap was observed in sensory and motor areas. The observed differences in functional variability for different specialized areas suggest that a more complete estimation of the structure-function relationship across the whole cortex requires further empirical studies with an expanded test battery.
\end{abstract}




\section{Introduction}

Despite the advances in functional neuroimaging of the last two decades a question which is central to cognitive neuroscience has remained largely unanswered; that is, to what extent do functional areas respect anatomical landmarks, or more generally, what is the relationship between structure and function in the human cerebral cortex? The creation of probabilistic maps based on the data from a large cohort of brains is probably the most powerful way to approach this question. One way to create probabilistic maps from a large sample of subjects is to utilise the already abundant data obtained in mapping experiments in different labs to carry out a meta-analysis. A metaanalytic approach however, would be sub-optimal as it would introduce unwanted sources of variability. Those sources are, among others, differences in the reporting of locus of activation due to different alignment and normalisation schemes, variability arising from different experimental paradigms and differences related to inconsistencies in analysis methods and thresholds used across studies (Thirion et al., 2007). In order to avoid these issues, we suggest the creation of data sets from a large cohort from which rich functional information is acquired using the same test battery and a consistent analysis pipeline.

\subsection{Spatial normalisation}

Spatial correspondence between subject's brains is a mandatory condition for meaningful whole brain group analysis as without accurate spatial correspondence, different cortical locations are compared across subjects as if they were the same area. Not accounting for inter-subject macro-anatomical variability is a major source of loss of statistical power in group statistics.

The most widely used normalisation procedures automatically morph an individual subject to fit a template brain. The two most commonly used volumetric normalisation procedures are a) transformation to Talairach space, based on the classic stereotactic atlas created by Talairach and Tournoux from one post-mortem examination (Talairach and Tournoux, 1980) or b) transformation to the MNI template space (Evans et al., 1993; Evans et al., 1992). 
Both the Talairach co-ordinate space and MNI template system have been extremely important tools to create spatial correspondences between subjects for group analysis. These normalisation techniques provide a means by which one can compare activated brain regions across studies as results can be reported in terms of a three dimensional co-ordinate system $(\mathrm{x}, \mathrm{y}$, and $\mathrm{z}$ position in $\mathrm{mm}$ ). Furthermore the widespread use of standardised co-ordinate frameworks allows for an efficient and practical system of reporting findings to the neuroimaging community and affords the possibility of creating vast databases (e.g. BrainMap.org). However a universal co-ordinate framework still does not exist making the creation of databases for the use of datamining and meta-analysis difficult (Derrfuss and Mar, 2009).

\section{2 (Macro-) Anatomical Correspondence}

Despite the many benefits of Talairach and MNI registration these normalisation procedures have severe drawbacks. The major problem being that the same coordinate can refer to different anatomical areas across subjects. Figure 1 shows two brains warped into Talairach space. One can see that, in the subject on the left, the co-ordinates $\mathrm{x}=-30 \mathrm{y}=-25 \mathrm{z}=54$ refer to a point on the anterior bank of the central sulcus whereas, in the second subject on the right, this same co-ordinate refers to an area on the posterior bank of the central sulcus. Even on visual inspection one can see that, despite being normalised to Talairach space, the brains still have very different cortical folding patterns. It is clear that even the most distinct macro-anatomical landmarks in the brain are often not brought into alignment after this normalisation procedure. This in turn has a detrimental effect on group statistics. 


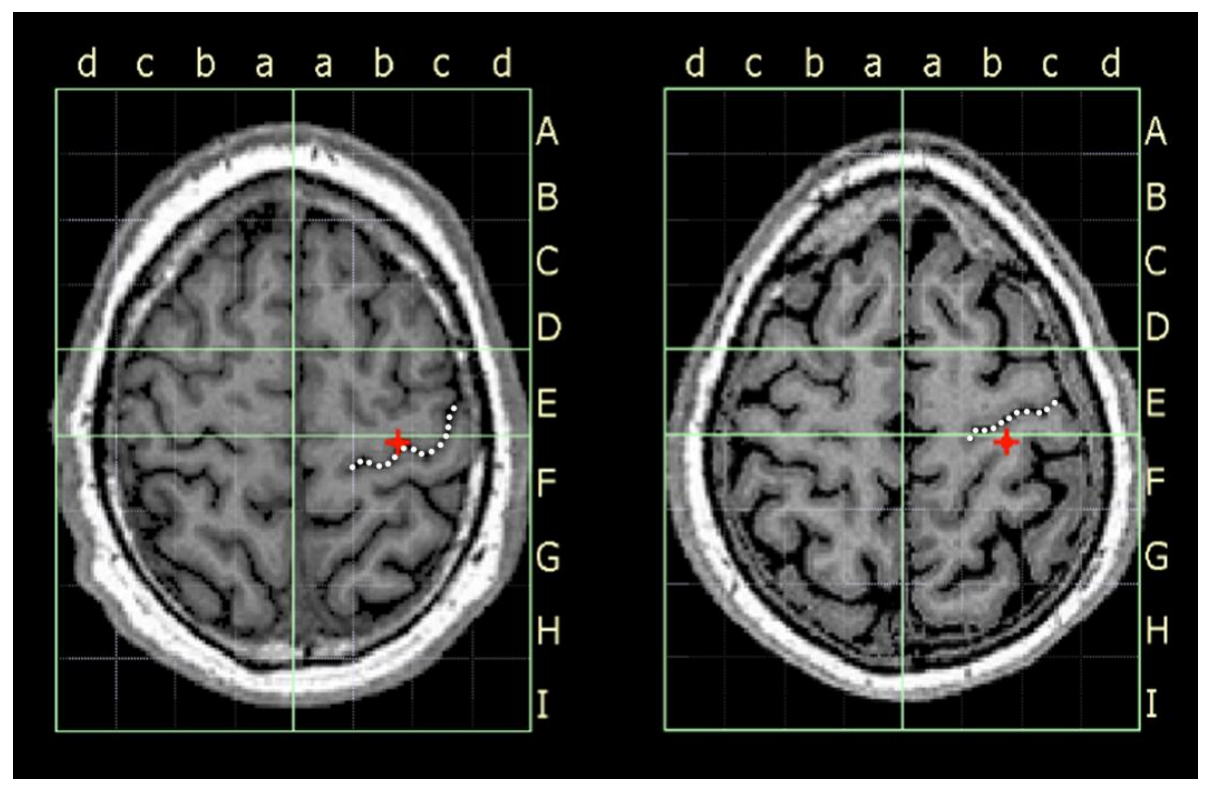

Figure 1. These two brains, which have been transformed into Talariach space, still exhibit unique cortical folding patterns. Talairach alignment is based on a few landmarks and is unable to co register individual macro-anatomy, i.e. gyri and sulci do not perfectly match across subjects; as a result, the same co-ordinate (used to average across subjects) can refer to a different anatomical area in each subject. Here the co-ordinates $x=30, y=-25, z=54$ correspond to a point on the anterior bank of the central sulcus in the brain on the left and a point on the posterior bank of the central sulcus in the brain on the right. The central sulcus is indicated by a dotted line.

The problem of macro-anatomical alignment lies in the fact that the human neocortex is essentially a two dimensional sheet, which is only a few millimetres thick. This cortical surface is highly folded to allow it to squeeze into the restricted cranial cavity and, although there are many consistencies across subjects, each individual has a unique sulcal and gyral folding pattern. The normalisation techniques described above that align the whole brain volume by keeping it as a three dimensional box and perform affine transformations are completely blind to these individual sulcal and gyral folding patterns and thus are only able to perform a 'global normalisation' or 'registration' of the data to a common space, yet are unable to actually "align" macro-anatomical features. While improvements of intensity-based volumetric alignment schemes have been proposed (Amunts and Zilles, 2001; Ashburner, 2007), approaches that explicitly align the folding pattern of the cortical surface (Fischl et al., 1999b; Goebel et al., 2006) or integrate volume 
and surface registration (Joshi et al., 2009; Joshi et al., 2007) appear more promising to obtain optimal macro-anatomical alignment.

\subsection{Surface-based cortex alignment}

The advantages of not limiting data analysis to volume space (3 dimensional) coordinate systems but to include surface (2 dimensional) representations have been known for over a decade. Pioneering work from a number of labs demonstrated the visualisation and analytic advantages of cortical surface reconstructions (Dale et al., 1999; DeYoe et al., 1996; Fischl et al., 1999a; Goebel et al., 1998; Sereno et al., 1995; Thompson and Toga, 1996; Van Essen, 2005; Wandell et al., 2000). The creation of flat maps and inflated hemispheres from surface reconstructions of the cortical sheet meant that activity in the entire cortex, including hidden sulcal regions, could be visualized on single, canonical representations reflecting the intrinsic topology of the cortex.

The shift to 2D surface representations of fMRI data also lead to advanced alignment procedures. Early approaches of macro-anatomical surface-based alignment were based on manual selection of landmarks (Van Essen, 2004, 2005; Van Essen et al., 1998). Fischl et al. showed that manual landmarks were not necessary for macro-anatomical alignment, instead, a local curvature-based optimization criterion was used on spherical cortex representations (Fischl et al., 1999b). The curvature-based alignment method demonstrated much improved macro-anatomical correspondence across brains and provided first evidence of improved concomitant alignment of histologically defined Brodman areas (Fischl et al., 2008). The use of such cortex based alignment (CBA) schemes has continued to grow and has been extended by transforming functional time course data directly in cortex space allowing one to carry out multi-subject general linear model (GLM) analysis and Independent Component Analysis (ICA) directly in cortex space (Formisano et al., 2004; Goebel et al., 2006) and to register brains to existing atlases (Yeo et al., 2010).

An important examination of the effectiveness and accuracy of surface based alignment techniques was carried out by Pantazis et al. (Pantazis et al., 2010). The authors compared the performance of landmark based registration, where a set of manually defined macro-anatomical landmarks act as targets of alignment, and curvature based registration, which automatically 
extracts curvature information from each individual cortical mesh and uses local curvature deviations to drive alignment.

Landmark based alignment techniques offer more flexibility than automatic curvature-based techniques which is important in some circumstances such as when carrying out lesion studies. Even among brains of healthy subjects, large variation in size, shape and orientation of gyri and sulci are difficult to reconcile using automatic curvature driven alignment. Furthermore the added flexibility of landmark based registration is very useful when one considers the fact that there is not always a one-to-one relationship between the number of sulci and gyri across subjects. For example, it is well known that there is variation in the number of transverse temporal gyri across subjects, with some subjects possessing a doubling of this structure (Leonard et al., 1998).

Landmark based alignment schemes also have disadvantages however. Firstly it is very time consuming to manually label landmarks. Secondly, to do so accurately requires a significant amount of anatomical knowledge and lastly, the personal interaction in the alignment introduces the possibility of subjective labelling influencing the resulting registration. In comparison, curvature based alignment is relatively fast; as long as one starts with high quality cortical surface mesh reconstructions an automatic alignment can be completed in a matter of minutes. Another clear benefit is that it does not require detailed anatomical knowledge and that it operates largely automatically. Furthermore curvature based alignment creates reproducible results with respect to a defined objective function leaving no room for experimenter bias and error to influence the alignment.

\subsection{Functional correspondence}

In order to investigate the relationship between structure and function in the brain, the current project evaluates the degree of spatial correspondence of functionally homologue specialized brain areas across subjects with respect to different anatomical alignment schemes. Spatial correspondence is quantified through the creation of probabilistic maps and assessment of the geodesic distance between homologue areas. Due to the fact that volume based registration techniques are unable to account for individual differences in cortical folding and assuming there is an inherent relationship between brain anatomy and brain function, we expect a higher degree of variation in the 
location of functional areas when brains are registered in volume space than when the same brains are registered with advanced surface driven techniques. More specifically, if advanced curvature/landmark-based alignment largely removes macro-anatomical variability (tested below) then the calculated probabilistic maps and distance measurements should largely reflect the true variability of the location of functionally defined homologue brain areas.

The common approach to solve the problem of poor overlap of functional regions when carrying out a whole-brain group study is to spatially smooth data, typically with a Gaussian kernel of $8 \mathrm{~mm}$ full width at half maximum. However as fMRI imaging techniques advance and data is acquired at an increasingly finer scale, smoothing in effect discards a vast amount of potentially useful data (Kriegeskorte et al., 2006). With an expected increase of spatial correspondence between functional areas across subjects, advanced surface based alignment techniques promise more powerful group statistics without the need to extensively smooth functional data. In a similar way that Fischl investigated the relationship between cortical folding and underlying cytoarchitecture (Fischl et al., 2008) the present study investigates the relationship between cortical folding and the location of functionally specialized brain areas.

In light of the potential issues of curvature-based cortical alignment (Pantazis et al., 2010) we first did a thorough investigation into our cortex based curvature alignment scheme and rigorously tested it to ensure that we significantly increased correspondence of macro-anatomical landmarks across subjects and that no major sulci were misaligned. Then functional probabilistic maps were calculated in volume (Talairach) space and aligned cortex (surface) space based on 10 subjects using 8 localisation experiments to label 13 functional areas. The obtained intriguing results motivate an extension of the project to create a freely available functional probabilistic atlas covering the whole cortex. 


\section{Materials and Methods}

Functional areas were localized in participants by using standard localising paradigms (see below for details). After defining specific functional regions in each subject we compared the spatial overlap of functional regions across subjects and geodesic distance of peak vertices between subjects, with respect to different normalisation/alignment schemes. All data analysis was carried out using the BrainVoyager QX v2.3 software package (Brain Innovation, Maastricht, The Netherlands).

\subsection{Participants}

10 right-handed subjects ( 3 females, 7 males) with a mean age of 30.3 (range 25-46 years) were scanned in 3 or 4 sessions each until the battery of 8 localising experiments had been completed for each subject. All subjects were right handed. Ethical approval was granted by the ethic committee of the Faculty of Psychology and Neuroscience at Maastricht University.

\subsection{Anatomical Alignment}

All anatomical volumes and functional time series were first warped into Talairach space. This operation included the definition of the landmarks AC (anterior commissure), PC (posterior commissure) and the borders of the cerebrum; the defined subject-specific landmarks were then used to rotate each brain in the AC-PC plane followed by piecewise, linear transformations to fit each brain in the common Talairach "proportional grid" system (Talairach and Tournoux, 1980). Then an automatic segmentation procedure was applied leading to the creation of topologically correct cortical surface reconstructions (Kriegeskorte and Goebel, 2001). Curvature maps, which are important for mesh morphing operations, were calculated on the folded representations. All hemispheres were inflated to a sphere and underwent, separately for the left and right hemispheres, a rigid-alignment cycle where curvature of each hemisphere was aligned to a randomly chosen target subject's curvature by global rotation. The rotation parameters that produced the highest overlap between the curvature of a subject's sphere and the target sphere were used as a starting point for subsequent non-rigid cortex-based alignment (CBA). 
CBA uses the extracted curvature information, reflecting the folding pattern, from each subject to create a group averaged curvature map on the sphere serving as the initial target curvature for the individual meshes. The CBA algorithm utilises curvature information as the local objective function and calculates gradient information to iteratively perform local vertex movements to increase curvature overlap. To increase robustness and accuracy, the CBA algorithm progresses from coarse to fine operating sequentially at four levels with different amounts of curvature smoothing. At the first level, individual curvature maps contain only the grossest anatomical features (central sulcus, Sylvian fissure, inter-parietal sulcus, superior temporal sulcus etc). Each subsequent level adds increasingly more anatomical detail until finally all curvature information from the folded cortices is included. The implemented algorithm is similar to the one described in Fischl et al. (1999) except that the target curvature map is calculated as the averaged curvature across all hemispheres at a given alignment stage (moving target approach, for details see Goebel et al., 2006). In this way CBA does not align each subject's curvature to a static template but to a dynamic group average that itself reflects the progress of macro-anatomical alignment.

In order to be able to ensure that CBA had improved alignment of macroanatomical landmarks, the fundus of 26 sulci (see figure 2) were labelled in each subject as had been done by Pantazis et al. (2010) in their investigation of the efficacy of alignment techniques. The following sulci were identified and their courses were traced following instructions found at http://neuroimage.usc.edu/CurveProtocol.html. 


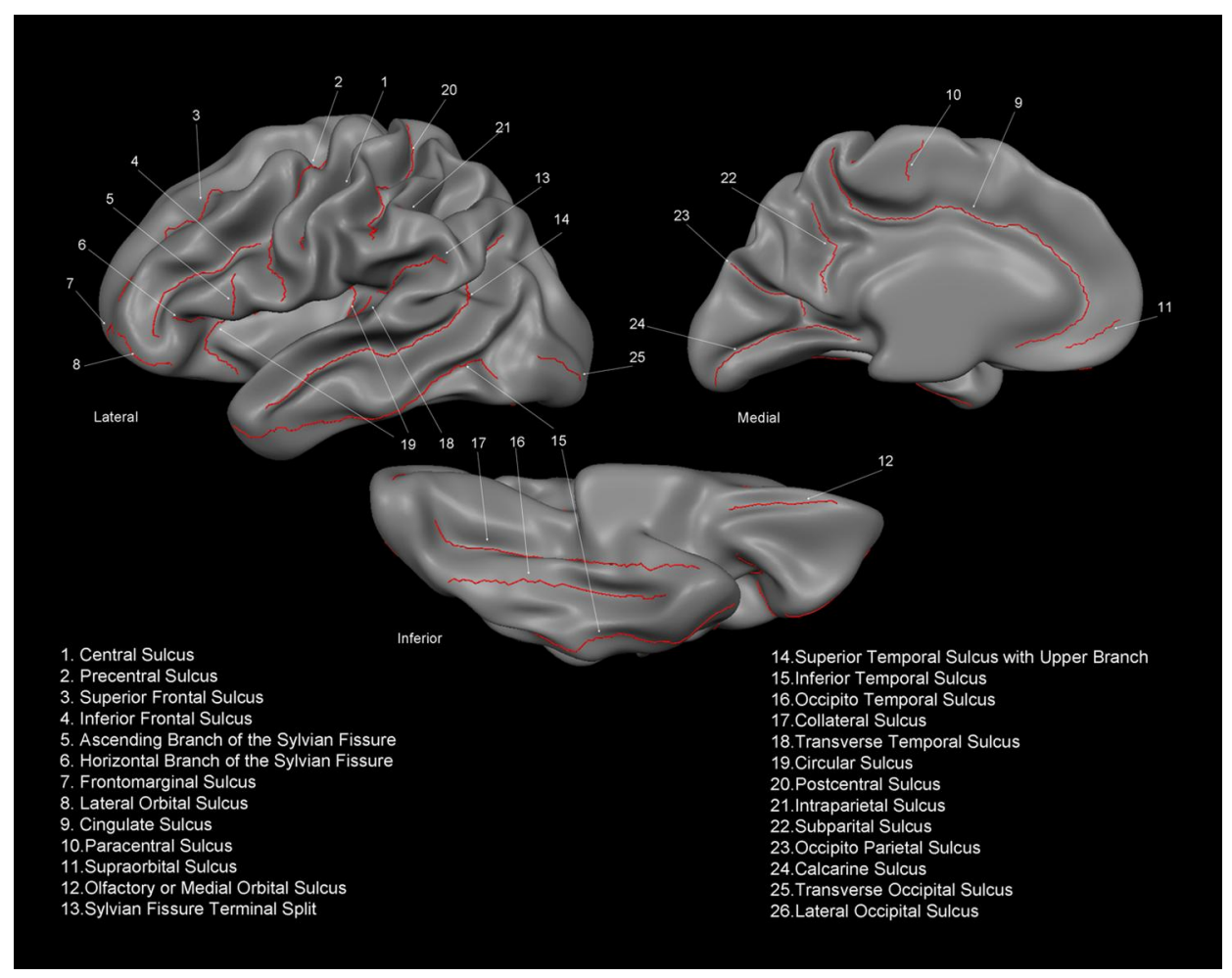

Figure 2. Outline of the macro-anatomical landmarks traced for each subject to evaluate the performance of curvature based alignment with respect to prominent landmarks (Pantazis et al., 2010). Note that the traced landmarks were not used during alignment; they served solely as a means to measure their inter-subject distance before and after cortex-based alignment.

To test the ability of CBA to align these landmarks we used a modified Hausdorff distance measure (Pantazis et al., 2010) to quantify the variance of pairwise path distances in the group for each landmark.

\subsection{Functional localisers - Area identification}

Thirteen functional regions of interest were defined by performing general linear model (GLM) analysis of time course data sampled on individual cortex meshes. In order to map fMRI signal time courses from volume space to surface space, values located between the grey/white matter boundary and up to $3 \mathrm{~mm}$ into grey matter towards the pial surface were sampled with trilinear interpolation and averaged resulting in a single value for each vertex of a cortex mesh. 
Functional areas of interest were determined by selecting the activity cluster on the surface falling in the region reported in previous standard localiser studies (see below). Once the resulting cortical patches of interest (POIs) have been marked and labelled it is possible to project the coordinates of a POI's vertices back into the anatomical volume in order to produce a corresponding functional volume-of-interest (VOI) in Talairach space. Finally the POIs defined in individual cortex space can be adjusted using the transformation parameters obtained from the CBA process, resulting in functional regions in macro-anatomically aligned cortex space. The overlap of POIs across subjects can thus, be compared between volume space and (aligned) cortical surface space.

Since in the outlined procedure the extent of identified clusters - and thus the overlap calculation - depends on subjectively determined thresholds, we also implemented an alternative, completely objective method. In this approach the location of the peak vertex within the active cluster of each subject is determined per area and the shortest distance of each subject's peak vertex to the mean location of the peak vertices is calculated. The shortest distances (geodesics) of the peak vertices to the group average location were calculated on the folded group mesh using the Dijkstra algorithm (e.g. (Cormen et al., 2001) Section 24.3: Dijkstra's algorithm. pp. 595-601). Since the distances of the peak vertices to the group average location are calculated before and after alignment, this method allows one to quantify, for each homologue area, how much CBA is able to increase spatial correspondence across subjects without requiring subject-specific adjustments of statistical thresholds.

\subsection{Functional localisers - task battery}

A battery of functional localisers was used to label functional regions across the cortex. Each POI was selected by labelling significantly active surface vertices guided by known anatomical locations. Areas in the ventral visual stream were identified using a standard localiser (Sorger et al., 2007), including the fusiform face area (FFA), parahippocampal place area (PPA) and dorsal caudal subdivision of lateral occipital cortex (LOC). The localising paradigm consisted of blocks of faces, houses, objects and scrambled objects presented for 16 seconds in a random order with every alternate block consisting of a rest period of central fixation. Each single stimulus was presented for 666ms with 17 milliseconds fixation in between each image. In 
all block types subjects were instructed to keep central fixation. The contrast used to localise FFA was "faces > houses". For PPA it was "houses > faces" and for LOC, "object > scrambled"

To localise V5/hMT+, an area sensitive to visual motion, a paradigm was used with two main conditions showing either 400 dots moving radially outwards from the centre of the screen or showing 400 stationary dots (Goebel et al., 1998). Four blocks of each experimental condition were presented to the subject and each alternate block contained a central fixation point and served as a block of rest. Subjects were instructed to keep central fixation during all block types. Area V5/hMT+ was identified using the contrast "moving dots > stationary dots". To functionally label the frontal eye fields (FEF), subjects were instructed to make saccadic eye movements to follow an empty white circle that moved to one of 8 possible locations on the horizontal and vertical meridian on the screen at a rate of $2 \mathrm{~Hz}$ for $16 \mathrm{sec}-$ onds. Each alternate block consisted of 16 seconds of a central fixation cross. The contrast used to label FEF was "saccades > fixation".

To localise functional areas within the sensory and motor networks we used a sequential finger tapping paradigm in which subjects learnt and repeated a sequence of finger tapping movements. Blocks commenced with the presentation of five boxes in the centre of the screen. Each box corresponded with a finger digit. Subjects were cued for the first $60 \mathrm{~ms}$ of each block as to which hand would be used during the block with the words "left hand" or "right hand" above the boxes. Following an $80 \mathrm{~ms}$ delay period 4 of the five boxes lit up in a sequence with each box illuminated for $50 \mathrm{~ms}$. The 2 second sequence was repeated, after an $80 \mathrm{~ms}$ delay, two more times so it was displayed to the subjects 3 times. During the presentation of the sequence the subjects tapped their fingers using the prescribed hand. The boxes then changed colour which cued the subject that their task was to repeat the sequence three more times without any further external cues but repeating the learnt sequence at the learnt pace. This was followed by a rest period of empty boxes which lasted 20 seconds. Following rest the next block commenced with the hand cue and continued with a new finger tapping sequence. In order to localise the pre-motor area (PMA) we contrasted learning phase and performance phase and labelled the most active vertices in the anatomically plausible region (i.e. anterior to the central sulcus and superior to the Sylvian fissure. To label the supplementary motor area (SMA) the contrast learning > performance was also used, however only the most active 
vertices on the medial surface of the hemisphere were labelled (Picard and Strick, 2001).

Using this paradigm it was impossible to differentiate between sensory and motor hand regions as the task involved both processes simultaneously. Activation anterior to the fundus of the central sulcus was labelled as hand motor area with activation posterior to the fundus of the central sulcus labelled hand sensory area.

We also employed a spatial processing / imagery task where subjects were instructed, to imagine navigating and walking around the university building when the fixation cross turned red. We chose the university building as it was very well known to each subject and provided a stimulating scene in which to navigate through. When the fixation cross turned green, subjects were asked to imagine 2-dimensional pictures, as if being shown a "national geographic-like" magazine. These were interleaved with rest periods of central fixation. All blocks were 16 seconds long. In order to label the precuneus, the contrast "spatial imagery > 2-dimensional imagery" was used and the most active vertices on the superior parietal lobe were labelled.

To activate the language system we devised a standard word generation paradigm. A single letter was presented centrally to the subjects for $20 \mathrm{sec}-$ onds and they were asked to silently generate words beginning with that letter. This was followed by a block of central fixation for an equal period of time and then a new experimental block commenced with the presentation of another letter. The contrast "word generation > rest" was used to locate two language related regions and thus labelled as "Broca's area" (anterior activations) and "Wernike's area" (posterior activations).

A cross modal region involved in the integration of input from the visual and auditory modalities was activated by simultaneous stimulation of both modalities. Sounds and images of animals were combined in 4 different ways; "congruent" blocks where the presentation of an animal image was paired with the presentation of the sound that animal makes, "incongruent" where an animal image was paired with the sound of a different animal, "images alone" where the images were presented without any accompanying auditory input and "sounds alone" where the animal noises were presented in the absence of visual stimuli (Hein et al., 2007). The contrast "congruent + incon- 
gruent $>$ images alone + sounds alone" was used to label a multi modal region on the superior temporal gyrus (STG).

\subsection{Quantifying POI overlap}

We computed the overlap of functional areas across subjects for vertices belonging to a POI of at least one subject using the formula:

$$
N_{v} / N
$$

Where $\mathrm{Nv}$ is the number of subjects whose functional area includes vertex $\mathrm{v}$ and $\mathrm{N}$ the number of subjects. The obtained values for all relevant vertices constitute the probabilistic map for a particular localised region-of-interest. An overall probabilistic map is obtained by adding the resulting overlap vertex values for all included POIs. A threshold of $>10 \%$ is applied to all probabilistic maps to avoid depicting regions as "overlap" where only one subject has a functional area.

\section{6 fMRI scanning parameters}

All subjects were scanned with a Siemens 3T head only scanner (Magnetom Allegra, Siemens Medical Systems, Erlangen, Germany). A standard echoplanar-imaging (EPI) sequence (repetition time [TR] $=2$ secs field of view $[\mathrm{FOV}]=224 \mathrm{~mm} \times 224 \mathrm{~mm}$, matrix size $=64 \times 64$, echo time $[\mathrm{TE}]=32$ $\mathrm{ms}$ ) with a voxel size of $3.5 \mathrm{~mm}^{3}$. Each volume consisted of 32 slices, covering the whole brain. A high-resolution structural scan (voxel size, $1 \times 1 \times 1$ $\mathrm{mm}^{3}$ ) was also collected for each subject (in each session) using a T1weighted three-dimensional (3D) ADNI sequence [TR, $2050 \mathrm{~ms}$; echo time (TE), $2.6 \mathrm{~ms} ; 192$ sagittal slices]. 


\section{Results}

\subsection{Macro-anatomical alignment results}

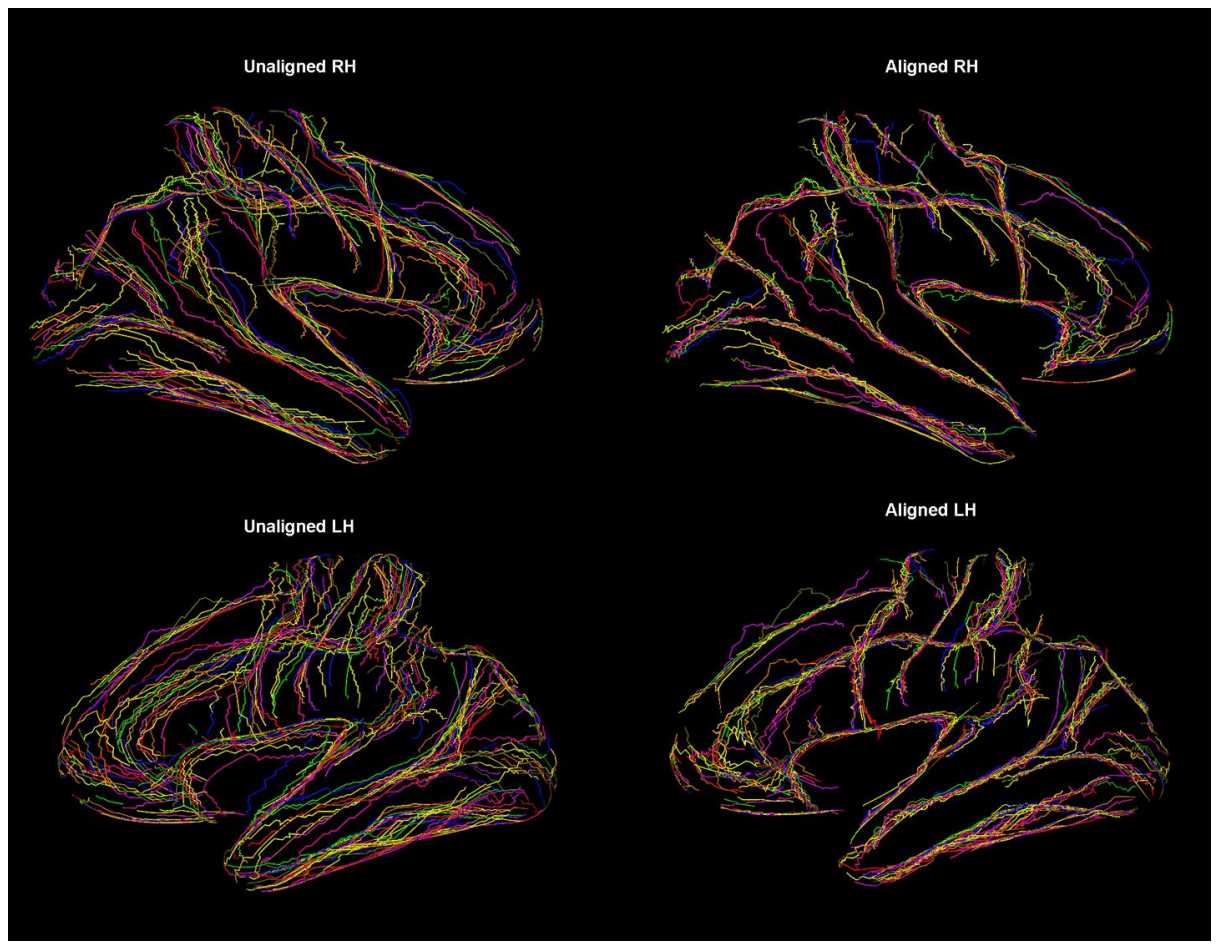

Figure 3. Sulcal landmarks in both hemispheres before CBA on the left and after CBA on the right. Each subject is represented by a different colour. We observe that same sulci across subjects are more clustered after curvature-driven alignment as well as an increased separation between different sulci. This indicates that curvature-driven alignment is able to bring major macro-anatomical landmarks into spatial correspondence.

CBA was successful in aligning most macro-anatomical landmarks. In the left hemisphere 22 of the 26 sulcal landmarks were brought closer together across subjects after CBA. In the right hemisphere 21 of the landmarks showed less variability across subjects after alignment. Landmarks with many interruptions (e.g. occipito-temporal sulcus) or which are very short (e.g. paracentral sulcus and lateral orbital sulcus) were those least well aligned (see Figs. 3 and 4). Note that all major landmarks (central sulcus, precentral sulcus, superior frontal sulcus, circular sulcus, cingulate sulcus, 
subparietal sulcus, calcarine sulcus, among others) were almost perfectly aligned, i.e. the standard deviation after CBA was smaller than $3 \mathrm{~mm}$. The central sulcus, calcarine, and pre-central sulcus were aligned with submillimeter precision.
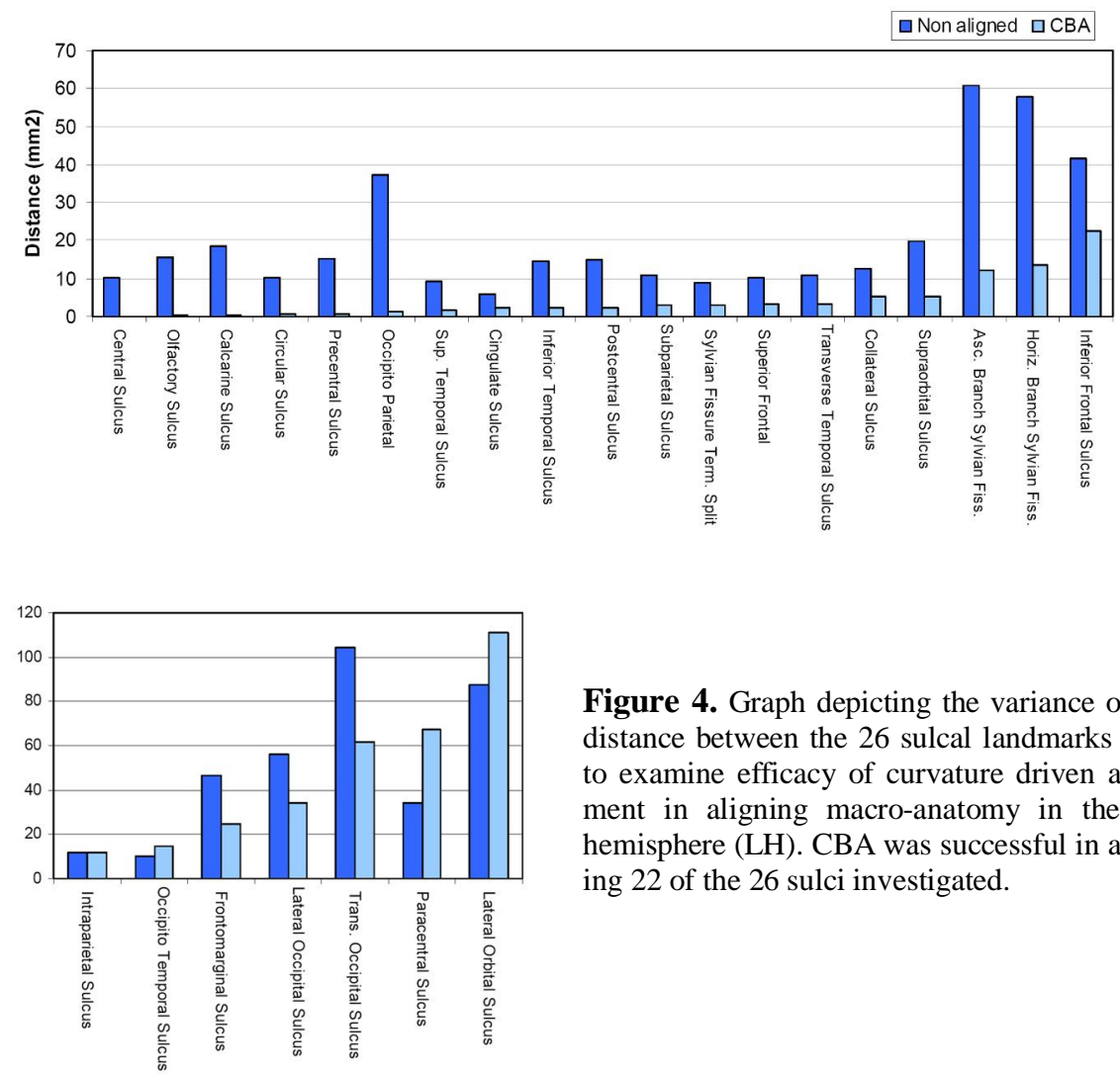

Figure 4. Graph depicting the variance of the distance between the 26 sulcal landmarks used to examine efficacy of curvature driven alignment in aligning macro-anatomy in the left hemisphere (LH). CBA was successful in aligning 22 of the 26 sulci investigated.

\subsection{Functional overlap results}

All included functional regions showed increased overlap in the probabilistic maps after CBA compared to volume based maps. However the percent gain in overlap differed greatly across the different functional regions throughout the cortex. There remained, for example, a large amount of variability in language regions in the left hemisphere (for overview see figure 5). Indeed there was only a modest gain in overlap across subjects, $8.35 \%$, when going from Talairach space, to aligned surface space in the language production 
area described here as Broca's region. The more posterior area activated by the language task, labelled "Wernike's area" showed a more substantial increase of $29.5 \%$ in overlap.

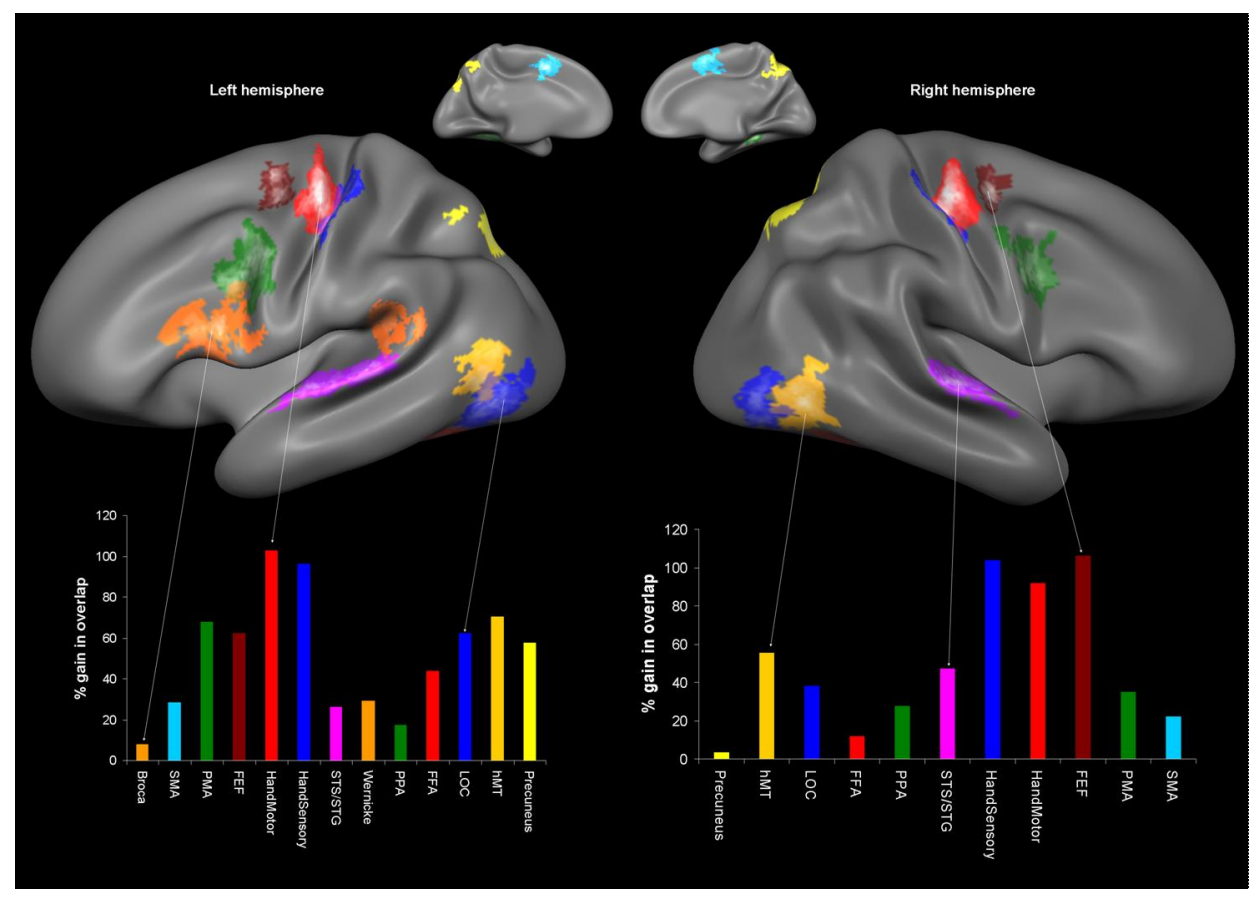

Figure 5. Probabilistic functional maps from across the cortex and histogram showing relative gain in overlap of functional areas after going from Talairach space to aligned cortex space. All areas show an increase in overlap, however the amount gained differs across the cortex.

Both the sensory and motor hand areas, located on either bank of the central sulcus were much better aligned after CBA. In the hand motor region there was a $102.9 \%$ gain in overlap in the left hemisphere and $91.9 \%$ gain in the same region in the right hemisphere. The hand sensory region also exhibit increased overlap with $103.9 \%$ increase in the right and $96.6 \%$ gain on the left hemisphere. Figure 6 shows that these two distinct functional regions are blurred together in Talairach space. Projecting the data on the surface increased the distinction between the areas but it is only after accounting for individual curvature patterns using CBA that these two functional regions become clearly separated. Other motor regions exhibited smaller gains. The SMA gained $22.4 \%$ in the right hemisphere and $28.8 \%$ on the left and PMA $35.1 \%$ on the right and $68.1 \%$ on the right. 


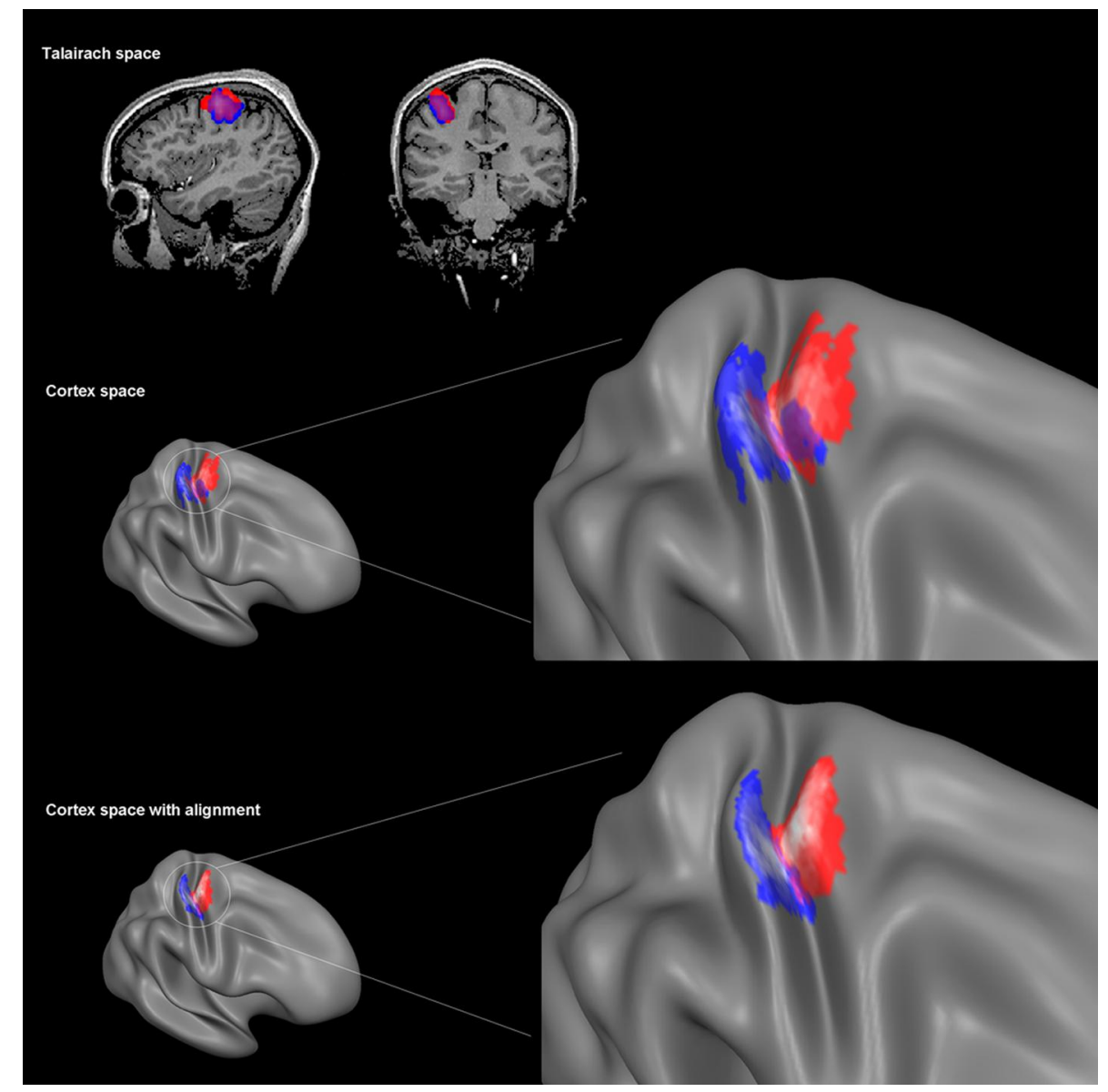

Figure 6. Probabilistic maps of the hand-motor (red) and hand-sensory (blue) areas in Talairach space (above) projected onto the surface (middle) and after curvature driven surface alignment (below). Brighter colour indicates increased overlap. 
Talairach space
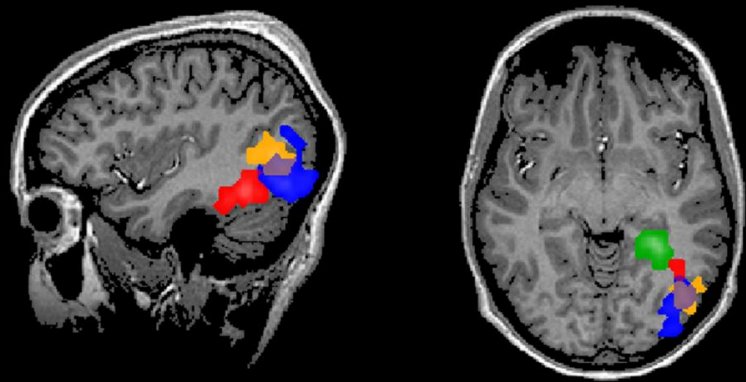

Cortex space

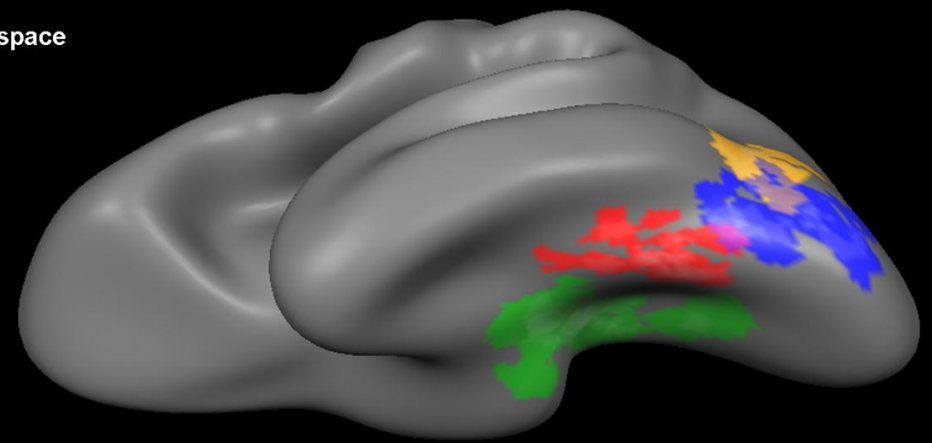

Cortex space with Alignment

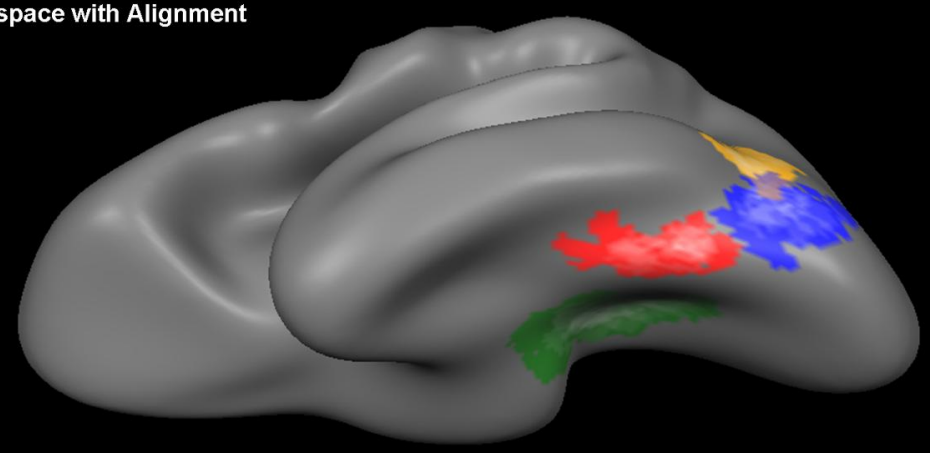

Figure 7. Zoomed view on probabilistic functional maps of major visual areas in Talairach space (top), non aligned surface space (middle) and CBA aligned cortex space (bottom). Green $=$ PPA, Red $=$ FFA, Blue $=$ LOC, Orange $=$ V5/hMT + . Brighter colour indicates increased overlap. 
Figure 7 highlights higher-level visual functional areas and shows the resulting increase in overlap of functional regions following CBA. Similar to results described above, CBA is able to separate functional regions which are more prone to blur together if individual anatomical curvature patterns are not accounted for. The area which shows the most gain in overlap of these regions is $\mathrm{V} 5 / \mathrm{hMT}+$ with $70.9 \%$ gain in the left hemisphere and $55.6 \%$ gain in the right hemisphere. Area LOC also showed increased overlap after CBA with a $62.7 \%$ gain in the left hemisphere and $38.4 \%$ on the right. Finally PPA exhibit more gain in the right hemisphere with $27.7 \%$ gain, than on the left with $17.6 \%$.

The precuneus, activated during the spatial navigation task, showed a surprising disparity of the extent of the gain obtained in overlap between volume and CBA space. On the right hemisphere there was a very small 3.7\% gain, while the left hemisphere showed $58 \%$ increase.

One of the most intriguing findings of a functional area that shows a strong correspondence with a macro-anatomical landmark is the frontal eye fields (FEF). This area has been shown to be involved in primate (Bruce and Goldberg, 1985) and human (Petit et al., 1997) saccadic eye movements. We consistently found an area of significant activation at the junction of the precentral sulcus and the superior frontal sulcus as described by Paus et al. (Paus, 1996). Overlap increased by $66.7 \%$ in the left hemisphere and $106.5 \%$ in the right hemisphere. Figure 8 shows the spatial extent of FEF in 10 subjects in the probabilistic functional map; the brighter the colour, the higher the number of subjects that contain FEF at the respective position (vertex). The left side shows the group overlap map before any cortex based macro-anatomical alignment. Although all 10 subject's FEFs occupy a coherent and anatomically plausible patch of cortex, there is not much overlap across subjects. This is indicated by the solid brown colour. The image on the right shows the same 10 subject's FEFs after the functional data has been transformed in aligned cortex space. It is clear that there is significantly more overlap after alignment with increasing overlap indicated by increasingly bright colours. The white colour at the centre of the patch indicates that FEF is located at this region in all subjects of the group. The image also depicts the sulcal paths; although these macro-anatomical landmarks were not explicitly aligned, curvature-based alignment has co-registered them successfully (different colours indicate landmarks of different subjects). Since macroanatomical variability has been almost completely removed in this location, 
the high degree of functional overlap suggests a tight correspondence between structure and function in this area.

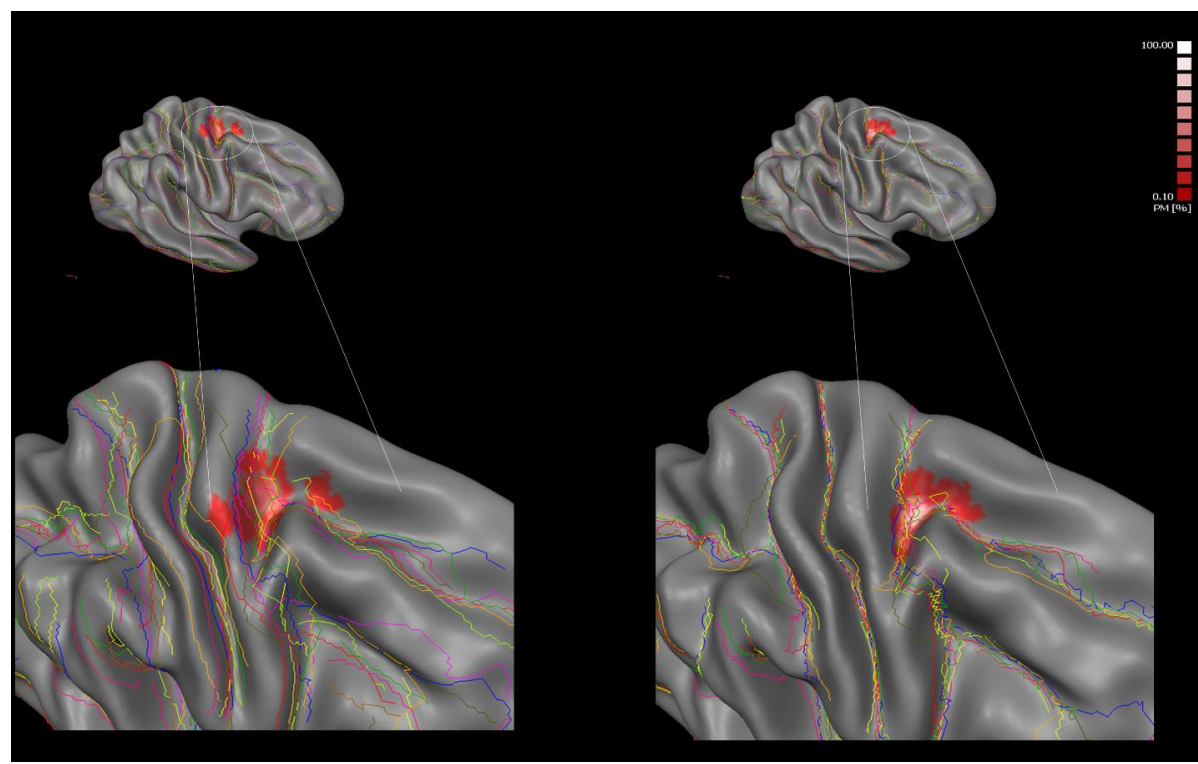

Figure 8. Frontal Eye Fields in 10 subjects. Left before CBA alignment, right after CBA alignment. There is much more overlap of the location of the functional area after macroanatomical alignment (seen with a brighter colour). This suggests that there is a strong relationship between structure and function in the FEF.

The Fusiform Face Area (FFA) on the other hand, does not exhibit the same strong structural - functional correspondence and saw more modest increases in overlap after macro-anatomical alignment with $44.1 \%$ and $12 \%$ gain for the left and right hemispheres, respectively. The FFA is a functional area which receives much attention due to its role in face processing (e.g. (Grill-Spector et al., 2004; Kanwisher et al., 1999; Tarr and Gauthier, 2000; Weiner and Grill-Spector, 2010). Its location showed a very interesting pattern across subjects. As seen in figure 9, there is much more inter-subject variability than in FEF. After curvature-driven CBA however, the differences mostly lie along the length of the fusiform gyrus and not in the direction perpendicular to that gyrus. 


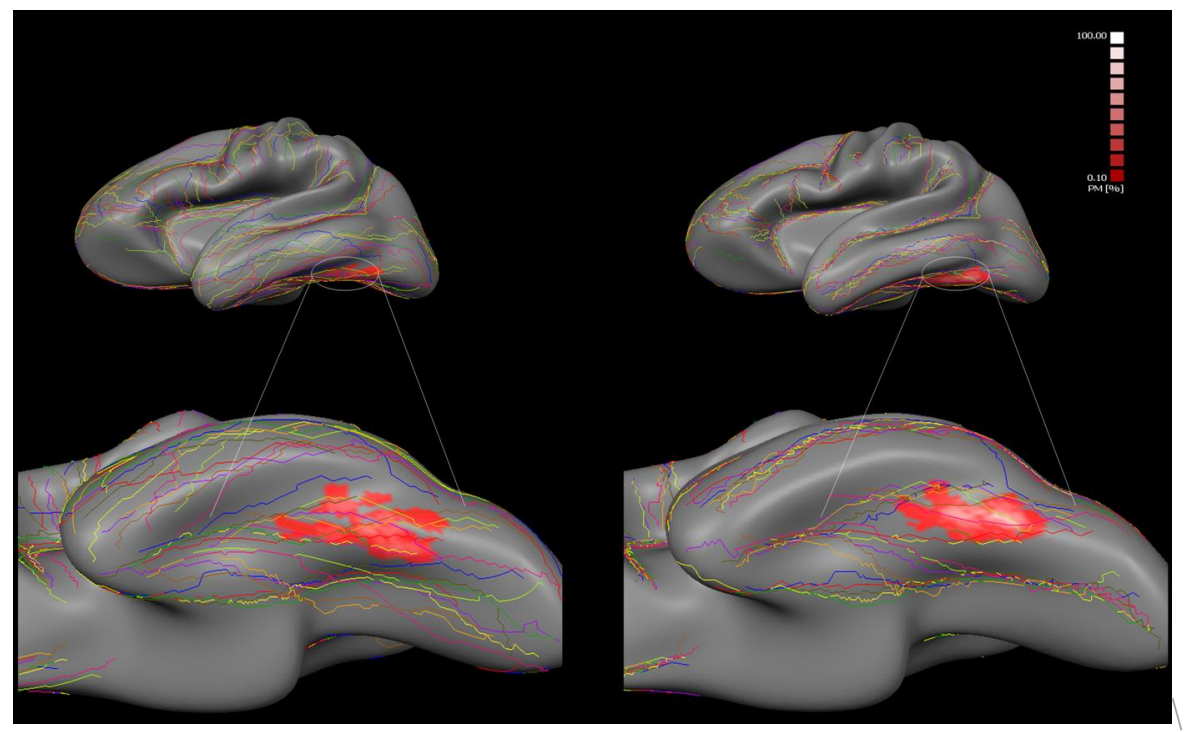

Figure 9. Probabilistic map of FFA before alignment (left) and after alignment (right) with sulcal paths superimposed. Anatomical alignment has brought the functional areas together however there still remains variability along the length of the gyrus.

\subsection{Peak vertex results}

In the left hemisphere all functional areas showed substantial reduction of the distance of the peak vertices to the respective group average (centre) location with FEF again exhibiting the largest gain from unaligned to aligned cortex space. Most functional areas in the right hemisphere also showed a reduction of the distance of the peak vertices to the respective group centre location, albeit less substantially. Two areas, PPA and SMA, exhibited a very slight increase in the mean distance to the group average location (see figure 10). 


\section{Left Hemipshere}

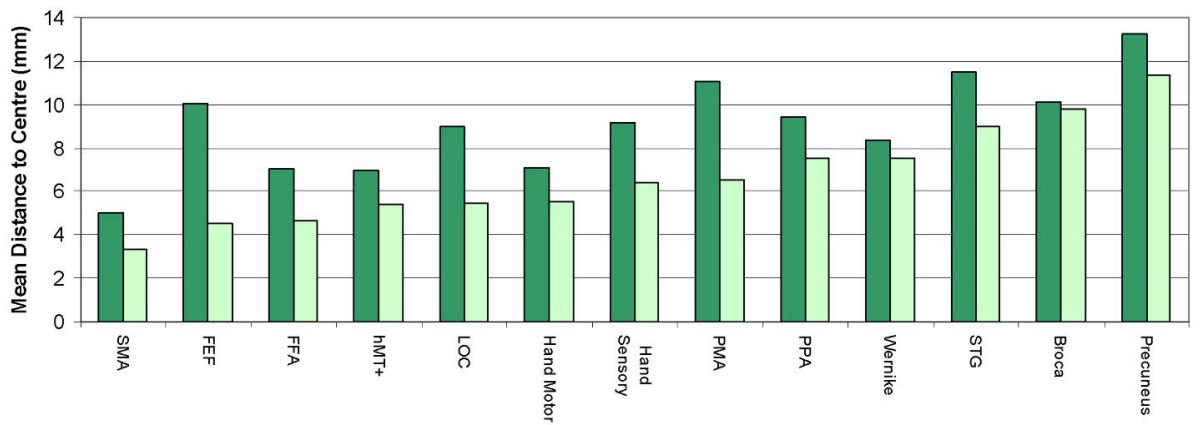

Right Hemipshere

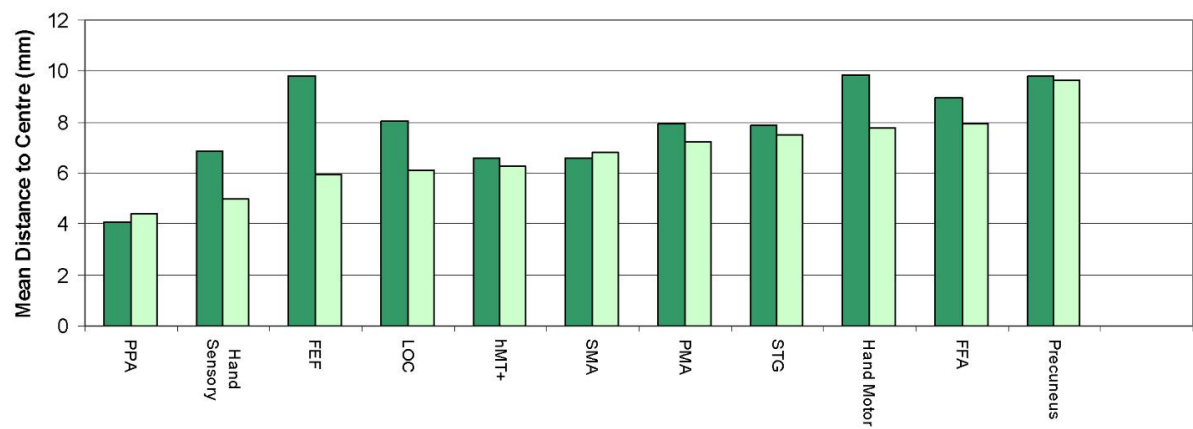

Figure 10. Histogram showing distance to the mean vertex location before and after alignment. Overall the peak active vertex of each subject showed movement towards the group mean after alignment the reflecting the finding the macro anatomical alignment results in concomitant improvements in the alignment of functional areas.

\section{Discussion}

By substantially reducing macro-anatomical variability of the cortex through curvature driven surface alignment we have been able to reveal the underlying relationship between structure and function for a number of specialised functional brain regions.

In order to investigate structural-functional correspondence through the creation of probabilistic functional maps, it is crucial to achieve whole brain macro-anatomical correspondence across subjects. Our careful examination of the efficacy of CBA has shown that automatic curvature based alignment 
techniques can align landmarks with very high accuracy. In fact, curvature driven alignment may even outperform landmark-based cortex alignment in achieving overall correspondence as alignment is not limited to a subset of regions (landmarks) but rather utilises curvature information from the entire cortical sheet to achieve alignment. It is imperative, however, that the results of an automatic alignment be examined and not just assumed to have produced an accurate group alignment. By assuring for each subject that the used curvature-driven CBA has correctly aligned all major macroanatomical landmarks, one can interpret the observed spatial variability of functional areas as mainly a measure of structural-functional relationship. Those sulci that did not seem to exhibit a reduction in variability after CBA were also those that were difficult to label, including very short landmarks (paracentral sulcus), or sulci located very close to parallel sulci (lateral orbital sulcus). It is thus possible that the macro-anatomy in these respective regions is, in actual fact, well aligned but this not reflected well due to mislabelling. This highlights the difficulty in labelling landmarks, especially those with multiple interruptions. The gain in overlap of functional areas that are located close to such landmarks are likely underestimating the true gain and should be considered preliminary. Our current set of functional areas were, however, located in regions where the surrounding macro-anatomy was aligned well (with the exception of Broca and, to some extent, FFA)

Our data show that there is a surprising amount of variability in that not all functional areas are tightly bound to anatomical landmarks, i.e. there is no general rule describing how macro-anatomical and functional areas correlate. Indeed in some regions there is a strong structural - functional correspondence while in others the spatial location of the functional area varies greatly across subjects within a cortical area. The FEF and FFA seem to be interesting examples of this observation. While it seems that CBA has brought the functional area FEF into alignment, there is a substantial amount of variability in FFA This remaining variability in the spatial location of FFA is likely to be caused by two factors. Firstly, because CBA aligns macro-anatomical curvature, there is much less alignment force along the length of a sulcus or gyrus (rather constant curvature values) than perpendicular to its extension (varying curvature values) leading to less good alignment of FFA along the fusiform gyrus. Indeed figure 11 shows that the anterior to posterior variability remains after CBA while the medial to lateral variability has been reduced substantially by macro-anatomical alignment. Another explanation for the remaining variability in the case of FFA may be that there is not one 
single area active in the fusiform gyrus when running a standard FFA localiser and that a different sub-region, activated by the FFA localising paradigm may be selected as "FFA" in different subjects (see also Weiner and GrillSpector, 2010). This would indicate that the observed variability along the fusiform gyrus reflects micro-anatomical (i.e. cytoarchitectonic) differences (Eickhoff et al., 2005) which are unobservable in vivo. Indeed published probabilistic cytoarchitectonic maps show similar variability in spatial extent and location of micro-anatomically defined areas. In particular the spatial location of BA 44, functionally defined as "Broca's area" has been shown to be highly variable (Amunts et al., 2004), even when individual curvature has been taken into account (Fischl et al., 2008) which supports our findings. A similar investigation into the variability of hOc5, thought to be the microanatomically defined area which is functionally known as hMT+, also shows a similar variability profile as the map of hMT+ in the present study (Malikovic et al., 2007).

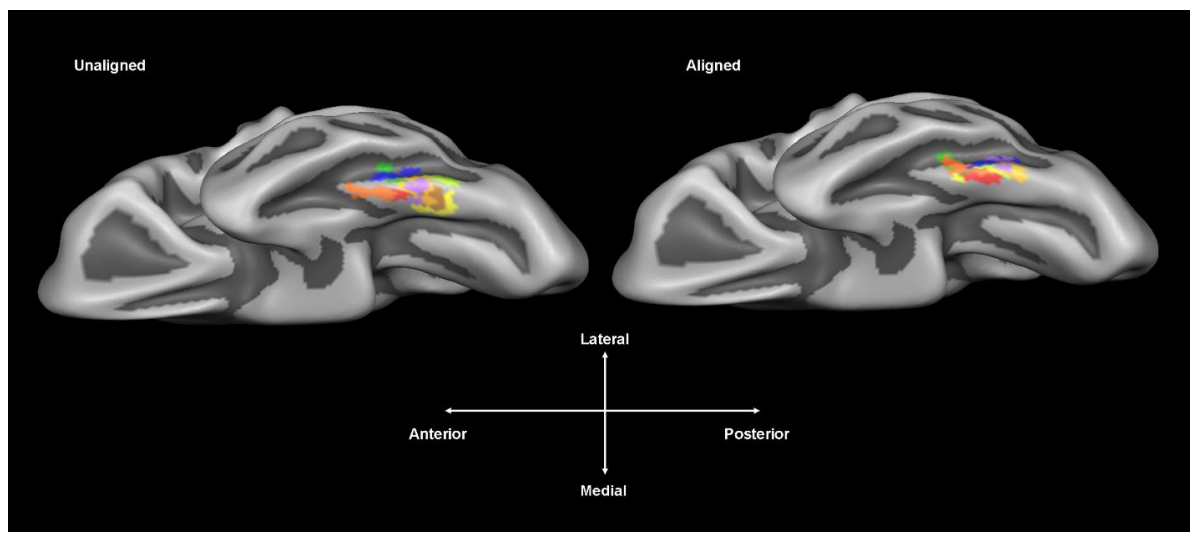

Figure 11. Fusiform Face Area in 10 subjects. Each colour represents FFA in one subject. Despite the fact that CBA has aligned the fusiform gyrus well in the medial - lateral direction, there still remain differences in the spatial location of the FFA along the length of the gyrus (anterior - posterior), where there is little alignment force. Macro-anatomical alignment alone is not capable of resolving these differences that are most likely to reflect differences of cytoarchitecture (i.e. different active regions might be labelled as "FFA").

We also observed greater overlap of functional areas (and stronger reduction of peak vertices to respective centre locations) in the left than in the right hemisphere. The number of subjects (10) is too small to draw definite conclusions about a strong asymmetry and the reasons of the observed difference. It might be that functional localizers do not work as well in the right hemisphere than in the left, or that macro-anatomical alignment does not 
work as well as in the left hemisphere, or that the structural-functional relationship is weaker in the right hemisphere.

By creating probabilistic functional maps using the same subjects for the investigation of all functional areas, the same scanner and the same analysis pipeline we have been able to gain important insights into the extent to which macro-anatomical landmarks are coupled to specific functional areas. A limitation of the current approach is that, due to individual differences in level of activation, it is impossible to use the same threshold for every subject. For this reason, great care has been taken to individually inspect all functional data and appropriately select the extent of each and every functional region. It appears however that region selection, as currently implemented, accurately reflects the underlying functional regions as the results of the gains in functional overlap are mirrored by the decreases in the distance of peak vertices to the group average location of an area. Since the latter measure is obtained without subjective choices, it provides converging evidence that macro-anatomical alignment substantially improves functional spatial correspondence in many areas.

One must also note that other sources of spatial variability may remain in the present probabilistic maps, one of which may be intra-subject variability. In short intra-subject variability is the slight variance in location of activation observed in one subject, under the same experimental conditions, across separate scanning sessions. It has been reported that intra-subject variability, as examined by a set of localizing paradigms of high level visual areas, was on a sub-voxel level (Peelen and Downing, 2005). More recently however Duncan (Duncan et al., 2009) indicated that the spatial location of functional areas can vary slightly within the same subject across sessions but notes that intra-subject variability can be minimized by using relaxed thresholds. The current study, by utilising robust localisers of well studied areas and not applying strict thresholds, should have kept intra-subject variability at a minimum according to these previous studies.

Our current study has important implications for statistical whole-brain analysis of group fMRI studies since our results indicate that functional correspondence is substantially improved in most investigated specialised areas. The application of an advanced surface-based macro-anatomical alignment scheme thus substantially increases statistical power beyond what is achievable with standard affine/piecewise linear volume normalisation techniques. 
It will be also interesting to assess in the future whether advanced volumetric normalization schemes (e.g. Ashburner 2007) lead to increased overlap of functionally defined specialized areas.

Despite substantial improvements, differences still remain in the location of functional regions after curvature-based alignment however, indicating that not all functional areas are tightly bound to anatomical landmarks (see figures 5, 8 and 9). Depending on the research question, it may be advantageous to forego anatomical accuracy and include functional landmarks as additional targets of alignment. Just as CBA uses curvature information as targets of alignment, functionally driven CBA (fCBA) would include functionally defined landmarks in the alignment procedure (Frost and Goebel, 2010). It must be noted however that fCBA would distort the anatomical information thus questions concerning the relationship between structure and function cannot incorporate this procedure, i.e. its use should be limited to further improve group analyses combining the advantages of whole-brain and ROI analyses. A similar procedure has been carried out using distributed networks as targets of multi-subject registration (Sabuncu et al., 2010). Registration based on functional data has also been used to create highly accurate and clear delineations of early visual areas (Larsson and Heeger, 2006) We hope that our present study, quantifying the structural-functional relationship in several areas located across the cortex, will help to reveal for which functional areas fCBA would be of benefit. So far it seems that areas such as FEF do not require this additional alignment step; however areas such as FFA would certainly benefit in cases where group spatial correspondence and improved statistical power is of more interest than anatomical accuracy.

\section{Conclusions}

Since our analysis suggests that there is no general level of structural - functional correspondence, it is important to map more functional brain regions. We will continue to not only add more functional areas to our map but also more subjects. With the resulting extensive database we will create a probabilistic functional atlas across the whole cortex that will be made freely available to the neuroimaging community in BrainVoyager format as well as in the GIFTI format (www.nitrc.org/projects/gifti) that is supported by all major software packages. Aside from basic insights in the structuralfunctional relationship of many specialised areas, such a probabilistic func- 
tional atlas may also serve as a useful tool to aid in labelling functional areas. 


\section{References}

Amunts, K., Weiss, P.H., Mohlberg, H., Pieperhoff, P., Eickhoff, S., Gurd, J.M., Marshall, J.C., Shah, N.J., Fink, G.R., Zilles, K., (2004). Analysis of neural mechanisms underlying verbal fluency in cytoarchitectonically defined stereotaxic space--the roles of Brodmann areas 44 and 45. Neuroimage $22,42-56$.

Amunts, K., Zilles, K., (2001). Advances in cytoarchitectonic mapping of the human cerebral cortex. Neuroimaging Clin N Am 11, 151-169, vii.

Ashburner, J., (2007). A fast diffeomorphic image registration algorithm. Neuroimage 38, 95-113.

Bruce, C.J., Goldberg, M.E. (1985). Primate frontal eye fields. I. Single neurons discharging before saccades. J Neurophysiol 53, 603-635.

Cormen, T.H., Leiserson, C.E., Rivest, R.L., Stein, C. (2001). Introduction to Algorithms, 2nd ed. ed. MIT Press and McGraw-Hill, Cambridge, Massachusetts.

Dale, A.M., Fischl, B., Sereno, M.I. (1999). Cortical surface-based analysis. I. Segmentation and surface reconstruction. Neuroimage 9, 179-194.

Derrfuss, J., Mar, R.A. (2009). Lost in localization: The need for a universal coordinate database. Neuroimage 48, 1-7.

DeYoe, E.A., Carman, G.J., Bandettini, P., Glickman, S., Wieser, J., Cox, R., Miller, D., Neitz, J. (1996). Mapping striate and extrastriate visual areas in human cerebral cortex. Proc Natl Acad Sci U S A 93, 2382-2386.

Duncan, K.J., Pattamadilok, C., Knierim, I., Devlin, J.T. (2009). Consistency and variability in functional localisers. Neuroimage 46, 1018-1026.

Eickhoff, S., Walters, N.B., Schleicher, A., Kril, J., Egan, G.F., Zilles, K., Watson, J.D., Amunts, K. (2005). High-resolution MRI reflects myeloarchi- 
tecture and cytoarchitecture of human cerebral cortex. Hum Brain Mapp 24, 206-215.

Evans, A.C., Collins, D.L., Mills, S.R., Brown, E.D., Kelly, R.L., Peters, T.M. (1993). 3D statistical neuroanatomical models from 305 MRI volumes. Nuclear Science Cymposium and Medical Imaging Conference, 1993., 1993 IEEE Conference Record, San Francisco, CA, USA, pp. 1813-1817.

Evans, A.C., Marrett, S., Neelin, P., Collins, L., Worsley, K., Dai, W., Milot, S., Meyer, E., Bub, D. (1992). Anatomical mapping of functional activation in stereotactic coordinate space. Neuroimage 1, 43-53.

Fischl, B., Rajendran, N., Busa, E., Augustinack, J., Hinds, O., Yeo, B.T., Mohlberg, H., Amunts, K., Zilles, K. (2008). Cortical folding patterns and predicting cytoarchitecture. Cereb Cortex 18, 1973-1980.

Fischl, B., Sereno, M.I., Dale, A.M. (1999a). Cortical surface-based analysis. II: Inflation, flattening, and a surface-based coordinate system. Neuroimage 9, 195-207.

Fischl, B., Sereno, M.I., Tootell, R.B., Dale, A.M. (1999b). High-resolution intersubject averaging and a coordinate system for the cortical surface. Hum Brain Mapp 8, 272-284.

Formisano, E., Esposito, F., Di Salle, F., Goebel, R. (2004). Cortex-based independent component analysis of fMRI time series. Magn Reson Imaging 22, 1493-1504.

Frost, M.A., Goebel, R. (2010). Using functional areas as additional targets for multi-subject cortex-based brain normalisation. 16th Annual Meeting of the Organization for Human Brain Mapping, Barcelona.

Goebel, R., Esposito, F., Formisano, E. (2006). Analysis of functional image analysis contest (FIAC) data with BrainVoyager QX: From single-subject to cortically aligned group general linear model analysis and self-organizing group independent component analysis. Hum Brain Mapp 27, 392-401. 
Goebel, R., Khorram-Sefat, D., Muckli, L., Hacker, H., Singer, W. (1998). The constructive nature of vision: direct evidence from functional magnetic resonance imaging studies of apparent motion and motion imagery. Eur $J$ Neurosci 10, 1563-1573.

Grill-Spector, K., Knout, N., Kanwisher, N. (2004). The fusiform face area subserves face perception, not generic within-category identification. Nat Neurosci 7, 555-562.

Hein, G., Doorman, O., Muller, N.G., Kaiser, J., Muckli, L., Namur, M.J. (2007). Object familiarity and semantic congruency modulate responses in cortical audiovisual integration areas. J Neurosci 27, 7881-7887.

Joshi, A., Leahy, R., Toga, A.W., Shattuck, D. (2009). A framework for brain registration via simultaneous surface and volume flow. Info Process Med Imaging 21, 576-588.

Joshi, A.A., Shattuck, D.W., Thompson, P.M., Leahy, R.M. (2007). Surfaceconstrained volumetric brain registration using harmonic mappings. IEEE Trans Med Imaging 26, 1657-1669.

Kanwisher, N., Stanley, D., Harris, A. (1999). The fusiform face area is selective for faces not animals. Neuroreport 10, 183-187.

Kriegeskorte, N., Goebel, R. (2001). An efficient algorithm for topologically correct segmentation of the cortical sheet in anatomical $\mathrm{mr}$ volumes. Neuroimage 14, 329-346.

Kriegeskorte, N., Goebel, R., Bandettini, P. (2006). Information-based functional brain mapping. Proc Natl Acad Sci U S A 103, 3863-3868.

Larsson, J., Heeger, D.J. (2006). Two retinotopic visual areas in human lateral occipital cortex. J Neurosci 26, 13128-13142. 
Leonard, C.M., Puranik, C., Kuldau, J.M., Lombardino, L.J. (1998). Normal variation in the frequency and location of human auditory cortex landmarks. Heschl's gyrus: where is it? Cereb Cortex 8, 397-406.

Malikovic, A., Amunts, K., Schleicher, A., Mohlberg, H., Eickhoff, S.B., Wilms, M., Palomero-Gallagher, N., Armstrong, E., Zilles, K. (2007). Cytoarchitectonic analysis of the human extrastriate cortex in the region of V5/MT+: a probabilistic, stereotaxic map of area hOc5. Cereb Cortex 17, $562-574$.

Pantazis, D., Joshi, A., Jiang, J., Shattuck, D.W., Bernstein, L.E., Damasio, H., Leahy, R.M. (2010). Comparison of landmark-based and automatic methods for cortical surface registration. Neuroimage 49, 2479-2493.

Paus, T. (1996). Location and function of the human frontal eye-field: a selective review. Neuropsychologia 34, 475-483.

Peelen, M.V., Downing, P.E. (2005). Within-subject reproducibility of category-specific visual activation with functional MRI. Hum Brain Mapp 25, 402-408.

Petit, L., Clark, V.P., Ingeholm, J., Haxby, J.V. (1997). Dissociation of saccade-related and pursuit-related activation in human frontal eye fields as revealed by fMRI. J Neurophysiol 77, 3386-3390.

Picard, N., Strick, P.L. (2001). Imaging the premotor areas. Curr Opin Neurobiol 11, 663-672.

Sabuncu, M.R., Singer, B.D., Conroy, B., Bryan, R.E., Ramadge, P.J., Haxby, J.V. (2010). Function-based intersubject alignment of human cortical anatomy. Cereb Cortex 20, 130-140.

Sereno, M.I., Dale, A.M., Reppas, J.B., Kwong, K.K., Belliveau, J.W., Brady, T.J., Rosen, B.R., Tootell, R.B. (1995). Borders of multiple visual areas in humans revealed by functional magnetic resonance imaging. Science 268, 889-893. 
Sorger, B., Goebel, R., Schiltz, C., Rossion, B. (2007). Understanding the functional neuroanatomy of acquired prosopagnosia. Neuroimage 35, 836852.

Talairach, J., Tournoux, P. (1980). Co-planar sterotaxic atlas of the human brain. Thieme, Stuttgart.

Tarr, M.J., Gauthier, I. (2000). FFA: a flexible fusiform area for subordinatelevel visual processing automatized by expertise. Nat Neurosci 3, 764-769.

Thirion, B., Pinel, P., Meriaux, S., Roche, A., Dehaene, S., Poline, J.B. (2007). Analysis of a large fMRI cohort: Statistical and methodological issues for group analyses. Neuroimage 35, 105-120.

Thompson, P., Toga, A.W. (1996). A surface-based technique for warping three-dimensional images of the brain. IEEE Trans Med Imaging 15, 402417.

Van Essen, D.C. (2004). Surface-based approaches to spatial localization and registration in primate cerebral cortex. Neuroimage 23 Suppl 1, S97107.

Van Essen, D.C. (2005). A Population-Average, Landmark- and Surfacebased (PALS) atlas of human cerebral cortex. Neuroimage 28, 635-662.

Van Essen, D.C., Drury, H.A., Joshi, S., Miller, M.I. (1998). Functional and structural mapping of human cerebral cortex: solutions are in the surfaces. Proc Natl Acad Sci U S A 95, 788-795.

Wandell, B.A., Chial, S., Backus, B.T. (2000). Visualization and measurement of the cortical surface. J Cogn Neurosci 12, 739-752.

Weiner, K.S., Grill-Spector, K. (2010). Sparsely-distributed organization of face and limb activations in human ventral temporal cortex. Neuroimage 52, 1559-1573. 
Yeo, B.T., Sabuncu, M.R., Vercauteren, T., Ayache, N., Fischl, B., Golland, P. (2010). Spherical demons: fast diffeomorphic landmark-free surface registration. IEEE Trans Med Imaging 29, 650-668. 


\section{CHAPTER 3}

\section{IMPROVED CORRESPONDENCE OF RESTING STATE NETWORKS AFTER MACRO-ANATOMICAL ALIGNMENT}

Corresponding publication:

Frost, M.A., Esposito, F and Goebel, R. (2012) Improved Correspondence of Resting State Networks after Macro-Anatomical Alignment. Human Brain Mapping. In Press. 


\begin{abstract}
Resting state brain activity, as measured with functional magnetic resonance imaging (fMRI) in the absence of stimulation, is currently investigated in clinical, pharmacological, developmental and cross-species neuroscience research. However, despite the general and wide interest in understating the nature of resting state networks (RSNs), there has not been a thorough investigation into the relationship between these functional networks and their adherence to underling brain anatomy. We acquired resting state fMRI data from 10 subjects and extracted individual and group RSN maps respectively using independent component analysis (ICA) and self organising group-level ICA (sogICA). Cortex based alignment (CBA), an advanced surface based alignment technique which uses individual curvature information to align individual subjects' brains to a dynamic group average, was used to maximise anatomical correspondence across subjects. Cross subject spatial correlations of the RSN maps (independent components) were carried out with and without CBA. Seven RSNs were identified amongst the most reported and studied ones, and, for all of them, we observed a systematic gain in the spatial correlation following CBA, although this was not uniform across RSNs. The observed increase in similarity of the functional RSNs after anatomical alignment illustrates that these functional networks are strongly related to underlying macroanatomical features. Moreover, our results demonstrate that by correcting for individual anatomical differences, advanced surface based alignment techniques increase the overlap of corresponding resting state networks across subjects, thereby providing a useful means to improve resting state group statistics with no need for substantial smoothing.
\end{abstract}




\section{Introduction}

Interest in the brain activity during a "task free" fMRI experiment has increased substantially in recent years. What started with a serendipitous finding that there is remarkable similarity in activation patterns while a subject is "at rest" (Raichle et al., 2001; Raichle and Snyder, 2007), has sparked research by a myriad of groups. Resting state brain activity is now investigated in clinical research (Mohammadi et al., 2009; Rombouts et al., 2005), developmental research (Fransson et al., 2007) and even cross-species research (Mantini et al., 2011; Rilling et al., 2007) amongst other fields. Despite the ever-increasing interest in resting-state activity and the default mode network (DMN) in particular, the degree to which functional resting-state networks (RSN) correspond with macro-anatomical landmarks has not been thoroughly investigated. Insights into this relationship are pertinent seeing that these intrinsic connectivity networks (ICN) are often defined based on their spatial proximity to cortical landmarks; in fact a seminal review of resting state research stated that the DMN is "anatomically defined" (Buckner et al., 2008). Besides the DMN, some of the most frequently investigated RSNs include fronto-parietal (FPN), auditory (AUD), sensory-motor (SMN), visual (VIS), superior-parietal (SPN) and self-referential (SRN) networks. Some recent work suggests that default mode activity may represent the brain cycling through its possible repertoire of configurations of these functional networks (Deco et al., 2011; Senden et al., 2012).

While the debate over the functional relevance of RSNs continues, methods to label and extract these networks continue to be developed. One of the simplest approaches used for mapping RSNs is seed-based cross-correlation, where a known node of the respective network is selected as a seed region of interest and all other nodes of the network are revealed through their linear correlation to the seed (Biswal et al., 1995). Another very popular approach uses independent component analysis (ICA) (Hyvarinen and Oya, 2000). ICA has been shown to be a useful method to apply to resting state data (see, e. g., van de Ven et al., 2004; Grecius et al., 2004; Damoisieaux et al., 2006; Mantini et al., 2007; Esposito et al., 2009), as it is a completely data-driven technique which is able to successfully separate several RSNs without the need to define seed regions. RSNs obtained by ICA, and, particularly the most widely studied RSN, the DMN, have been revealed with a high degree of within- and between-subject consistency (Damoiseaux et al., 2006; De 
Luca et al., 2005; Esposito et al., 2009; Mantini et al., 2007; Meindl et al., 2010, Smith et al., 2009).

Previous work addressing the question of the relationship between structure and function of RSNs has mostly focused on measures of anatomical connectivity. The spatially separate nodes of resting state networks are, by definition, functionally connected, however this does not necessarily imply that there are direct anatomical connections between the nodes of the network. There have been a number of studies that have investigated whether these functionally connected regions are also anatomically connected via fibre tracts, as reconstructed by diffusion tensor imaging (Damoiseaux and Greicius, 2009; van den Heuvel et al., 2009). The evidence from these studies generally indicates that these functionally connected resting state networks are also structurally connected but not in all cases, for example no direct anatomical connections could be found between two of regions of the DMN (Greicius et al., 2009).

Our approach to examining structural-functional relationships focuses on investigating the consistency of networks with respect to macro-anatomical landmarks. After largely removing macro-anatomical variability, a gain in the overlap of RSNs across subjects is expected when these functional networks "respect" anatomical landmarks. In order to evaluate this we employ a surface-based, curvature-driven alignment technique subsequently called cortex-based alignment (CBA), to largely reduce macro-anatomical variability across subjects (Fischl et al., 1999; Goebel et al., 2006). CBA utilises the geometric properties inherent in the convoluted human neo-cortex and uses individual convex/concave curvature gradients to align the gyral/sulcal folding pattern across subjects. It has previously been shown that advanced macro-anatomical alignment procedures can lead to increased spatial correspondence of functional areas (Argall et al., 2006; Fischl et al., 1999; Frost and Goebel, 2012).

Recently we showed that, by reducing inter-subject macro-anatomical variability through curvature driven alignment, a concomitant reduction in spatial variability of discrete functional areas is observed indicating the degree of cortical structure-function relationship (Frost and Goebel, 2012). The present paper uses the same logic but applies it to RSN functional connectivity distributions as obtained by ICA decompositions and cortical projections of these component maps as opposed to discrete specialised functional 
areas (e.g. fusiform face area, parahippocampal place area, frontal eye fields etc). This paper examines the spatial variability of resting state networks in healthy subjects, before and after advanced macro-anatomical alignment. We expect that inter-subject spatial variability of RSN cortical maps will be reduced following macro-anatomical alignment, resulting in more sensitive group statistics over RSN cortical maps. We expect, however, that the potential gains in the inter-subject spatial similarities of the networks will be relatively modest (compared to, e.g., task-evoked regionally focused activations). In fact, since RSN activity is normally highly distributed over the entire cortex, any increase in overlap of the RSN layouts will mainly be a reflection of alterations occurring at the "fringe" (border) of these distributions, i.e. in a small fraction of the overall network pattern.

\section{Materials and Methods}

\subsection{Participants}

10 healthy subjects ( 4 female, 6 male) with a mean age of 31.3 (range 25-46) were scanned as part of a larger project (Frost and Goebel, 2012).

\subsection{Data acquisition}

242 volumes of resting state data were acquired after 30 mins of functional scans consisting of visual area localisers, the data of which is not included in the present paper. Participants were instructed to close their eyes but remain awake. The projection screen was darkened to provide a relaxing environment, free of all visual stimuli. All participants confirmed that they did not fall asleep during the resting-state scan.

\section{3 fMRI scanning parameters}

All subjects were scanned with a Siemens 3T head only scanner (Magnetom Allegra, Siemens Medical Systems, Erlangen, Germany). A standard echoplanar-imaging (EPI) sequence (repetition time $[\mathrm{TR}]=2 \mathrm{~s}$ field of view $[\mathrm{FOV}]=224 \mathrm{~mm} \times 224 \mathrm{~mm}$, matrix size $=64 \times 64$, echo time $[\mathrm{TE}]=32 \mathrm{~ms}$ ) with a voxel size of $3.5 \mathrm{~mm}^{3}$. Each volume consisted of 32 slices, covering the whole brain. A high-resolution structural scan (voxel size, $1 \times 1 \times 1$ $\mathrm{mm}^{3}$ ) was also collected for each subject using a T1-weighted three- 
dimensional (3D) ADNI sequence [TR, $2050 \mathrm{~ms}$; echo time (TE), $2.6 \mathrm{~ms}$; 192 sagittal slices].

\subsection{Data Analysis}

Analysis was carried out using the BrainVoyager QX v2.3 software package (Brain Innovation, Maastricht, The Netherlands).

\subsubsection{Functional pre-processing}

The first two functional volumes of the resting state scan were removed to allow T1 saturation to stabilise. The remaining 240 volumes underwent a series of pre-processing steps. Slice scan time correction was performed using sinc interpolation to correct for the interleaved slice acquisition. Motion correction was performed using a 3D rigid-body transformation of each volume to the first volume: motion was first detected using trilinear interpolation and then corrected using sinc interpolation. To remove scanner related linear and non-linear drifts a temporal high-pass filter with cut-off set to 3 cycles per time-course was applied. Functional images were normalised in Talairach space. Finally, data was spatially smoothed, using a Gaussian kernel of 5mm FWHM and temporally smoothed with a Gaussian kernel of 2 sec FWHM.

\subsubsection{Anatomical pre-processing and alignment}

High-resolution T1 weighted images of each participant were normalised in Talairach space. This operation included the definition of the landmarks AC (anterior commissure), PC (posterior commissure) and the borders of the cerebrum; the defined subject-specific landmarks were then used to rotate each brain in the AC-PC plane followed by piecewise, linear transformations to fit each brain in the common Talairach "proportional grid" system (Talairach and Tournoux, 1980). Cortical surface representations were created using an automatic segmentation tool (Kriegeskorte and Goebel, 2001). Individual curvature maps were then derived from the extracted cortical meshes. Cortex based alignment (CBA) was then performed by first carrying out a rigid alignment to a randomly chosen target subject, followed by a nonlinear morphing alignment to a dynamic group average curvature map. This proceeds from a coarse to fine level where, during the early phase, smoothed 
curvature maps are used to align the gross anatomical features (Central sulcus, Sylvian fissure, cingulate sulcus etc); as the alignment algorithm progresses, more detailed curvature information is included to maximise macroanatomical correspondence across the whole cortex (Frost and Goebel, 2012; Goebel et al., 2006). CBA results in the creation of files describing the nonlinear mapping of each subject's reconstructed cortex from normalized (Talairach) subject space, to the macro-anatomically aligned group space. These vertex-level mapping files are also used to transform the functional data (individual RS maps) to the group aligned space.

\subsubsection{Single subject and group ICA}

The pre-processed and Talairach normalised resting state data for each individual subject was analysed using a standard independent component analysis (ICA) technique, which has been shown to be a robust data driven method for extracting resting state networks (van de Ven et al., 2004). A plug-in for the BrainVoyager QX 2.3 software (Brain Innovation, Maastricht University, Maastricht, The Netherlands), which implements the fastICA algorithm (Hyvarinen and Oja, 2000) was used. Before ICA was performed, principal component analysis was applied to reduce data dimensionality to 30 (Esposito and Goebel, 2011). The ICA algorithm then computed 30 spatially independent components. Another plug-in, self organizing group ICA ( $\operatorname{sog}$ ICA), was used to automatically match and cluster components across subjects based on linear correlations between components (Esposito et al., 2005). As the time-courses of any two components from different resting state scans can not be assumed in phase, we only used the spatial similarities (computed as the correlations between the ICA maps and not the temporal similarities, i.e. e. the correlations between the ICA time-courses). In more detail, sogICA implements a hierarchical clustering algorithm which operates with the constraint that each subject has to contribute one component per cluster. Here, all 300 components (30 per subject, 10 subjects) were clustered, resulting in 30 clusters. In order to assess the quality of the produced clusters, the within-cluster similarities and the cluster "silhouettes" (Rousseeuw, 1987) were calculated and plotted for the selected clusters. The cluster silhouettes allow one to assess how much a given member is "wellclustered", with higher positive values pointing to little doubt about its assignment and lower negative values pointing to a possible misclassification. 


\subsubsection{Statistical analysis}

After ICA maps were extracted from the individual resting state data and components matched across subjects, a linear correlation analysis was carried out to measure spatial similarity across subjects after projection of the RSN maps onto a common cortical space. To this end, seven individual ICA maps, from those clusters comprising the most studied RSNs, were taken from each subject and projected to the individual cortical surface. From the individual surface, the RSN maps of each subject were then transferred to a common average cortical surface using a point-by-point correspondence, and spatially correlated with the homologue RSN maps from all other subjects undergoing the same cortical mapping procedure. These correlations, as well as their mean and standard deviation across all subjects, were calculated for both un-aligned and cortex based aligned common spaces, using all cortical map points on the "average" surface mesh, amounting to $~ 80.000$ spatial observations. Aligned surface maps were created using the output of the CBA algorithm to transform the RS network maps to the macroanatomically aligned space.

In order to assess the stability of the obtained results, a slightly different approach was additionally used, where each network map of each subject was correlated with the group average network map in both aligned and nonaligned surface space. For display purposes, group RSN cortical maps were obtained from 1-sample t-statistic maps in the common cortical space using both un-aligned and cortex-based aligned maps, and thresholded at $\mathrm{p}=0.05$ (uncorrected). 

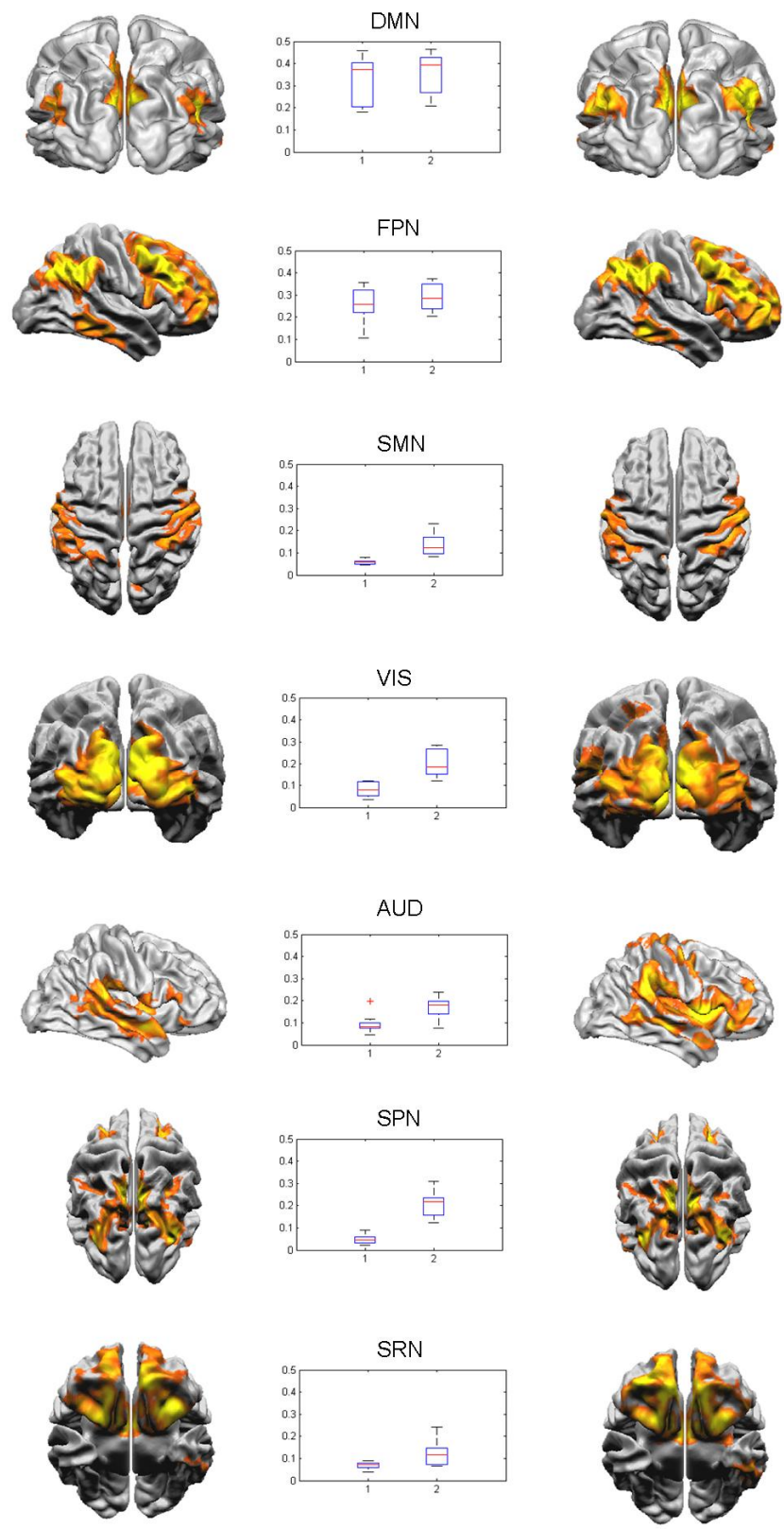

Figure 1. An overview of the 7 resting state networks. Group maps are shown unaligned on the left and aligned with CBA on the right. The box plots display the correlation values of the spatial similarity between subjects in both unaligned and CB-aligned space The central plots to the left (1) display correlations before alignment and the plots to the right (2) display correlations after alignment. 


\section{Results}

From the sogICA analysis, seven RSN components were identified that were highly similar to those reported in previous ICA-based RS-fMRI studies. These RSN components could be functionally categorized by the Talairach coordinates of the most active sub-regions (for reference, see, e.g., the table presented by Allen et al. (2011)) and were labeled as DMN, FPN, AUD, SMN, VIS, SPN and SRN (Damoiseaux et al., 2006; Mantini et al., 2007). Figure 1 gives an overview of the 7 RSN group maps obtained without and with CBA as well as cross-subject correlations of the RSN maps before and after alignment. All other clusters were discarded as their component members mostly contained artefact or noise components as well as lower silhouette values indicating suboptimal clustering (see also Supporting Information A).

In the subsequent correlation analyses, we observed a generalised increase in the correlation of all RSNs after the application of CBA (see Fig. 2). The subject-wise correlation, obtained by correlating the maps of each subject to all other subjects, of the DMN maps increased from 0.33 without to 0.35 after alignment whereas that of the FPN maps increased from 0.25 to 0.29 . These gains, however, were modest and not statistically significant ( $p>0.05)$. The average correlation of SMN maps increased from 0.06 to 0.14 . The VIS component maps increased from an average correlation of 0.08 to 0.20 . AUD maps increased from 0.09 to 0.16 . The SPN maps displayed the largest gain from 0.05 before alignment to 0.21 after alignment. Finally the SRN maps increased from an average correlation of 0.07 to 0.12. Apart from the DMN and FPN cases, all these gains were statistically significant $(\mathrm{p}<0.05)$.

Figure 3 reports the silhouette plots of the 7 RSN maps before and after alignment. It indicates that the cluster qualities improved for all RSNs and all subjects with only a few exceptions concerning the least representative subjects. 


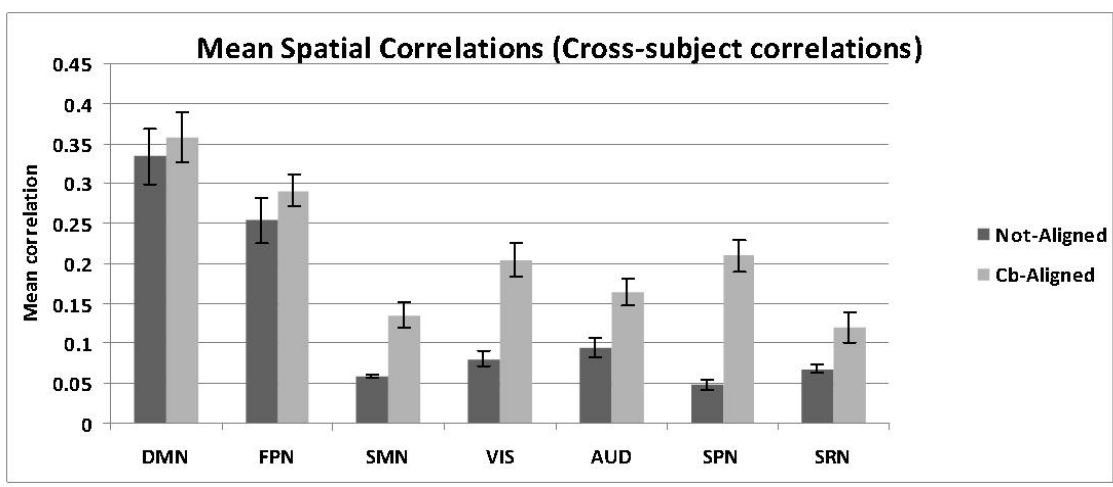

Figure 2. Bar graph depicting the average correlation values for 7 resting state networks. Correlations were computed by correlating the map of each network of each subject to the same network of all other subjects in a pair-wise manner. This was done in both non-aligned and cortex based aligned surface space.
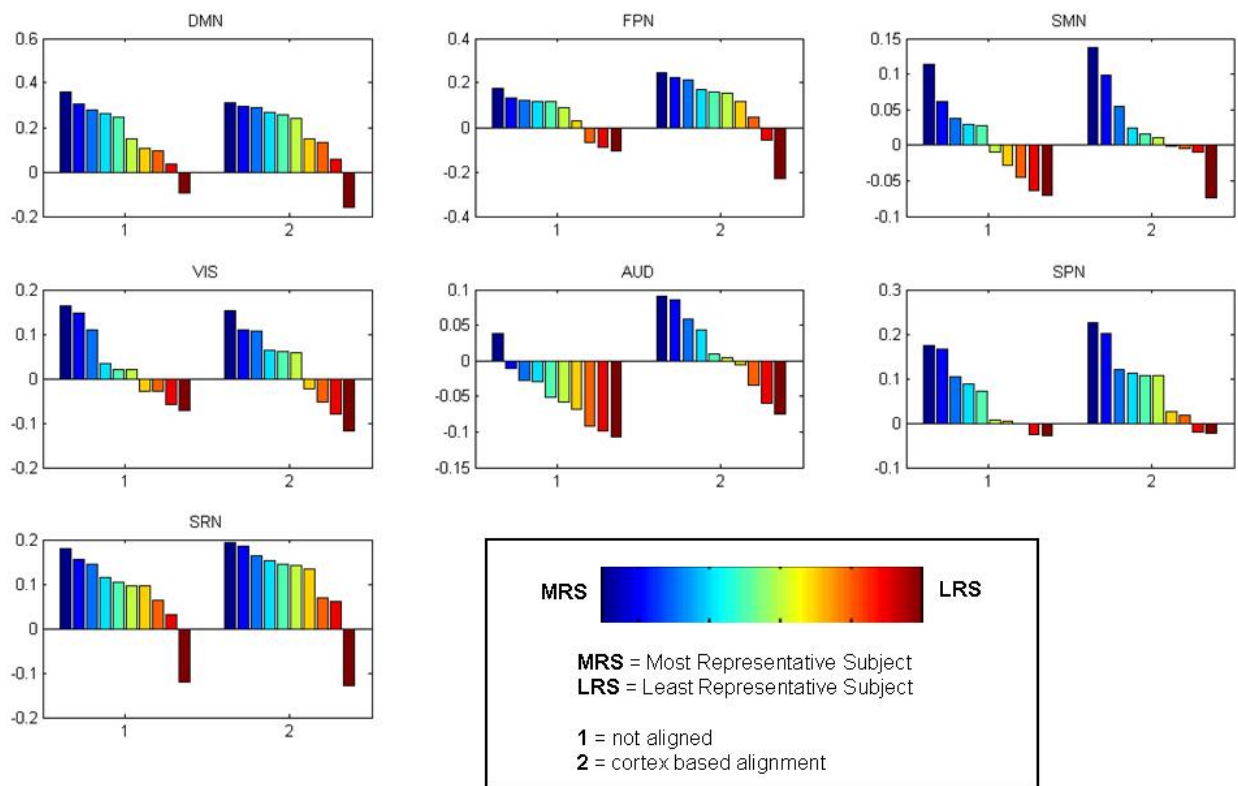

Figure 3. Plots of the Silhouette plots of the clusters where higher values indicate better clustering and lower values indicate possible mis-clustering. Silhouette values are higher after CBA (2) than without curvature alignment (1).

When correlation coefficients were calculated examining the spatial similarity of each subject's individual network maps with the group average of that network, we also observed an increase in similarity, albeit on a more modest scale. Table 1 shows mean correlation levels for all RSNs based on this ap- 
proach. Obviously, when correlating an individual's map to the group average, the correlation levels were much higher independent of the alignment approach. For instance, the smallest correlation value (obtained for the AUD component unaligned $=0.44$ ) was still higher than the strongest correlation of subject to subject correlations (DMN aligned $=0.35$ ). Also the difference between the non aligned and aligned conditions was diminished when compared to the cross-subject correlations. However, 6 of the 7 RSNs (with the sole exception of SRN) still resulted in being more highly correlated to the group mean after alignment than before alignment thus suggesting the same systematic albeit modest effect of CBA increases on the spatial similarity of the RSNs.

Table 1.

\begin{tabular}{|c|c|c|}
\hline RSN & Not-Aligned & CB-Aligned \\
\hline DMN & 0.63 & 0.65 \\
FPN & 0.54 & 0.60 \\
SMN & 0.45 & 0.46 \\
VIS & 0.47 & 0.53 \\
AUD & 0.44 & 0.47 \\
SPN & 0.51 & 0.54 \\
SRN & 0.45 & 0.45 \\
\hline
\end{tabular}

Table 1. Displays mean correlation values of individual subject maps to group average maps of the same resting state network

Despite the fact that visual differences between the group RSN maps before and after alignment are subtle, on close inspection one can see the effect of cortical alignment on these maps. Figure 4 shows two group RSN maps, the SPN and the AUD network, both are shown unaligned and aligned using CBA. Some clear differences are visible between the maps of the AUD network such as the gain in group level "activation" revealed through more areas showing stronger values (brighter yellow) after CBA. This is coupled with an increased spatial coherency of the functional maps after anatomical alignment, most obviously characterised with more focused activation in the superior temporal sulcus and the angular gyrus. One can see in the AUD network that the improvement is visible in planum temporale and the superior temporal sulcus, which are two secondary auditory regions crucial for auditory processing (Belin et al., 2002; Binder et al., 2000). As for the SPN, 
the improvement is most visible in the lateral intra-parietal sulcus, a region involved in saccadic eye movement control and imagery and spatial attention (Corbetta, 1998; Culham et al., 2001).

\section{Discussion}

Using the combination of single-subject ICA to extract individual RSN maps, and self-organizing group ICA, to match RSN maps and cluster them into groups, we were able to extract the functional maps of the 7 most consistent and widely studied RSNs in all our subjects. All 7 resting state networks examined in this study exhibited higher inter-subject correlations and better clustering after CBA application. This can be interpreted as evidence that the RSNs are intrinsically bound to the underlying macro-anatomical cortical boundaries and that macro-anatomical (cortex-based) alignment coregisters RSNs better than standard volumetric normalization. More specifically, by performing a purely anatomically (curvature) driven alignment, we observed systematic, albeit weak, gains in the spatial similarity of the RSN maps across subjects.

Not all of the networks, however, saw the same gain in similarity when comparing spatial correlation coefficients between the non-aligned and the aligned network maps. In fact, the RSNs with strongest (pre-alignment) inter-subject correlation benefited least from CBA and, at the same time, the added value of CBA was most evident among the RSNs with lower SNR, which makes detailed effects of alignment difficult to interpret.

The spatially most consistent RSN was the DMN. Along with its clearly pronounced spatial profile, easily distinguishable on visual inspection, the DMN is the RSN which has the strongest structural-functional relationship, in other words, the functionally defined DMN is highly related to macroanatomical boundaries, i.e. cingulate sulcus, angular gyrus, superior temporal sulcus, occipito-parietal sulcus. Interestingly, while the DMN was also ranked as the "most similar" network in terms of spatial correlations, both before and after alignment, we observed that it gained the least in terms of increase in correlation of the network maps. One must note that, even before applying CBA, the DMN is more highly correlated than any other resting state network map. The absence of a large gain in correlation after macroanatomical alignment is likely due to the high baseline (i.e. volumetric pre- 
alignment) level of inter-subject correlation. On the other hand, this could be also due to the fact that, compared to other RSNs, the DMN maps are less noisy in, and less variable across, all subjects, thereby reducing the net gain of anatomical alignment.

Unaligned

A.

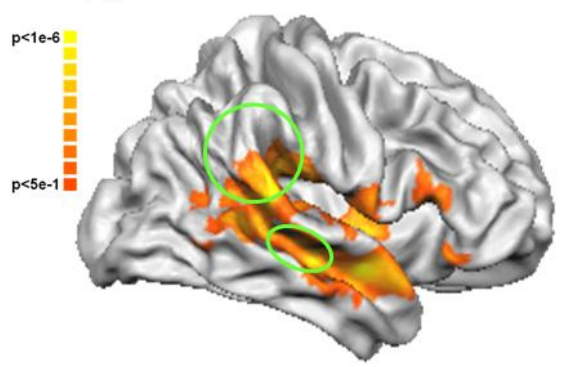

C.

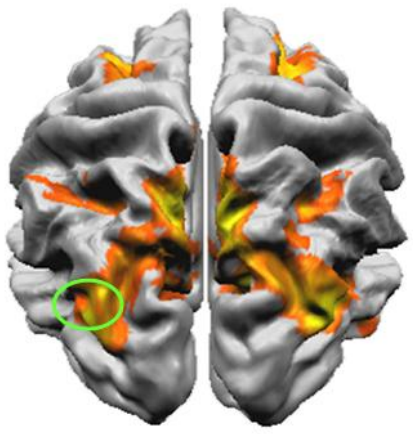

CB - Aligned

B.
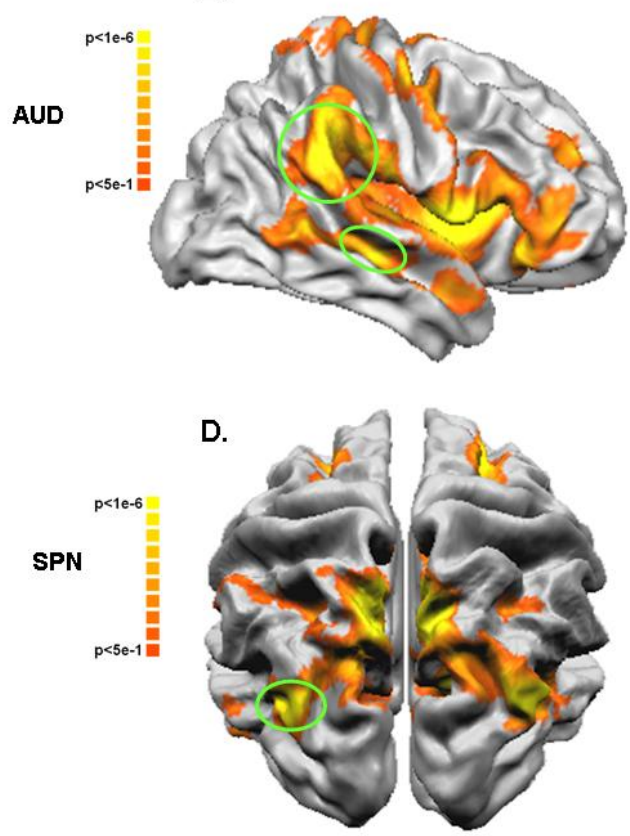

Figure 4. Group maps of the AUD and the SPN RSNs. On the left are the unaligned group maps of the AUD RSN (A.) and the SPN (C.). On the right is the group map of the AUD RSN after CBA (B.) and SPN (D.) also after the data were aligned using CBA. Key differences between aligned and non aligned maps have been highlighted in circles. For the AUD and the angular gyrus and superior temporal sulcus sees the clearest sharpening and for the SPN maps a clear gain is seen in the superior parietal lobule

It is interesting to note that the two RSNs which had the most significant gains in spatial cross-subject correlation were the SPN and VIS network maps. The parietal and occipital lobes in particular are known to have high variability in the organisation and orientation of sulci and gyri, such as the calcarine sulcus, lateral occipital sulcus and the intra parietal sulcus, in stan- 
dardised (Talairach) space across subjects (Frost and Goebel, 2012; Perrot et al., 2011; Thompson and Toga, 1996). By applying the CBA algorithm, thereby effectively minimising this inter-subject macro-anatomical variability, we observed a significant increase in the spatial similarity of these RSN maps, as measured by increased spatial correlation between all subject pairs. That we were able to reduce the spatial variability in these two RSNs, whose underlying anatomies are highly variable, is further evidence that the functionally defined maps are intrinsically related to the underlying anatomical structure.

We observed a large difference between correlations of subjects when they are correlated to the group average map of each RSN compared to when these are computed in a pairwise fashion to the map of each other subject. This finding is easily understood as the mean network maps already contain information from each subject. A subsequent correlation of a single subject to that group will, of course be higher than a correlation to another individual subject. It is striking however that even for correlations to the group mean map we observe a systematic improvement after CBA although the gain in spatial correlation between non-aligned and CBA aligned maps is modest. Furthermore, the construction of a group average network map, by its very nature, does not reveal areas of inter-subject variability but reflects the most consistent areas across subjects. The goal of this research however is to investigate to what extent this inter-subject variability is due to differences in individual macro-anatomy. By focusing on the difference between nonaligned and aligned cross-subject correlations we can observe the effect of the morphology of the underlying anatomy on the spatial layout of functional networks. As we observed post alignment gains across all the studied networks it seems that RSNs correspond to cortical macro-anatomy.

Due to the distributed and widespread nature of RSN maps it is difficult to observe the correspondence between functional activations and their adherence to underlying macro anatomical structures (sulci and gyri) in the maps themselves. Moreover, as a consequence of these aspects, gains in overlap of functional maps are strongest at the fringes of these maps and, therefore, visual differences can be quite subtle. Nonetheless clear examples can be found where the gains in spatial correspondence, as measured by increases in spatial correlation, are coupled with visible differences on the functional network maps. Two such examples, the SPN and the AUD network, demonstrate that, after anatomical alignment, the functional maps not only gain in 
group level activation strength but also the improved spatial correspondence of anatomy leads to increased 'sharpening' of these two functional maps. This is most clearly observed by the increased group level 'adhesion' to underlying anatomical boundaries such as the superior parietal lobule in the case of the SPN and the angular gyrus and superior temporal sulcus for the AUD network maps (See Fig. 4). It should be made clear however that, owing to the fact that we are concerned here with functional networks and not discrete functional areas, this "sharpening" of the activated RSNs cannot always be expected to accompany increased spatial correspondence. In contrast to task-based fMRI, where the activity in a given region depends only on the signal in that region, RSNs represent a distributed functional connectivity, thereby more or less "activity" in a given region depends not only on the signal in that region but also on the signal in other regions of the network.

It is also important to note here that, as well as the sharpening of functional maps and a decrease in the "extent" of the activity due to better macroanatomical alignment, we also observed other instances of increased spatial correlation in the absence of a shrinking of the extent of the activated network which might be expected. This is due to the intrinsically distributed nature of the RSN patterns. As spatial correlations were computed over the entire cortical space, including both "more involved" and "less involved" cortical regions, while any improvement in pattern similarity by CBA would only result in an increase in the average spatial correlations, the spatial extent of this activity (as obtained by setting a threshold to the group maps) can show two seemingly opposing effects. Firstly with an increase in the average spatial correlations, the spatial extent of this activity may become more focal and the extent therefore decreases as the distance between activated anatomical features is decreased through alignment. On the other hand minimising macro-anatomical alignment also allows more vertices to surpass the threshold and therefore increase the extent of the network.

The findings presented here mirror our previous work, which investigated the relationship between structure and function of specialised areas in the cortex using fMRI (Frost and Goebel, 2012). Whereas in that study we focused on examining the effect of macro-anatomical alignment on discrete functional areas as typically considered for a region of interest analysis (fusiform face area, hMT+, lateral occipital cortex, frontal eye fields, Broca's area etc) here we have focused on the relationship between structure and 
function in widespread, distributed functional networks. A similar pattern of results have emerged in both data sets. Firstly that, in the majority of cases, a reduction in anatomical variability has resulted in an increase in spatial similarity of functional areas/networks. Secondly, not all areas or networks benefit from macro-anatomical alignment to the same extent suggesting that the degree of structural-functional correspondence is not uniform across the cortex.

It is worth noting that the size of the inter-subject correlations was modest, and that, even when significantly increased after cortical alignment, these remained small, suggesting that only a minimal fraction of the total variance in the RSN patterns can be ascribed to the anatomical variability. In general, there are several factors of functional, physiological and demographic nature, which, beside anatomical variability, could equally explain the intersubject variance of the RSN patterns for example, the individual levels of previous music exposure (Kay et al., 2012; Luo et al., 2012) motor exercise (Vahdat et al., 2011) and creativity (Takeuchi et al., 2012), as well as the caffeine (Rack-Gomer et al., 2009) and alcohol (Esposito et al., 2010) blood concentrations, and the different genders (Filippi et al., 2012). It is indeed the highly "plastic" behaviour of the RSN patterns that has made their study attractive in the clinical research.

The results of the present study provide some interesting insights into how one might improve group statistics. It is clear in any group brain imaging study that there is a reduction in spatial sensitivity stemming from the fundamental differences in cortical folding patterns across subjects. While a common technique used to account for this unwanted variability for functional averaging is to utilise spatial smoothing kernels, this approach sacrifices spatial resolution, which is not tolerable for high-resolution studies that are increasingly performed in the context of both ultra-high field acquisition and multivariate pattern analysis (see, for example. De Martino, 2011).

Our results presented here illustrate that a minimal but distinct gain in spatial overlap can be achieved in group studies by improving the macro-anatomical correspondence across subjects. Furthermore our results demonstrate that the used surface based, curvature driven alignment technique effectively increases spatial similarity of the 7 major RSNs considered in this paper. This is strong evidence that these intrinsic functional networks are systematically related to specific macro-anatomical structures. 


\section{Supplementary Materials}

\section{Supporting Information A}
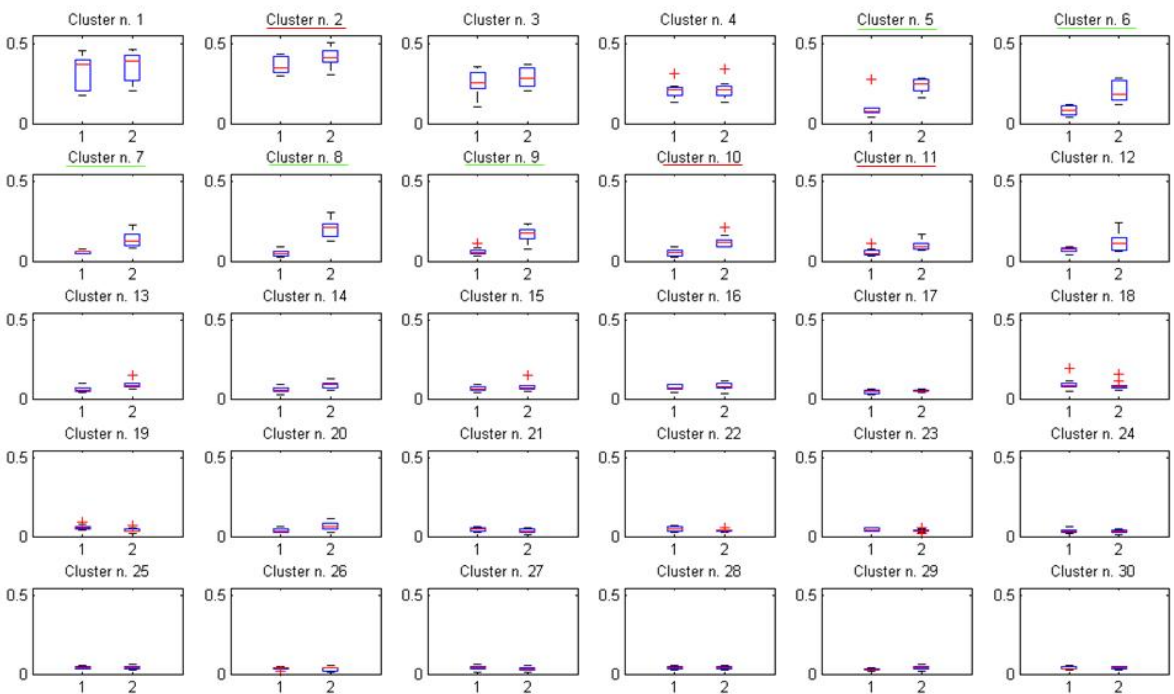

Supporting Information A. Comparison of changes in similarity of clusters from nonaligned (1) (Talairach, normalised, surface space) to cortex-based aligned surface space (2). Highlighted in green are RSN clusters with a significant difference in cluster similarity between aligned and non-aligned data. Highlighted in red are significant non-RSN cluster similarity changes from volume-only to cortex-based alignment. This figure shows that 5 of the 7 RSNs were significantly more similar after CBA than they were after volumetric normalization (Talairach space). Only 3 of the remaining 23 non-RSN clusters were significantly more similar after CBA. 


\section{Supporting Information B}

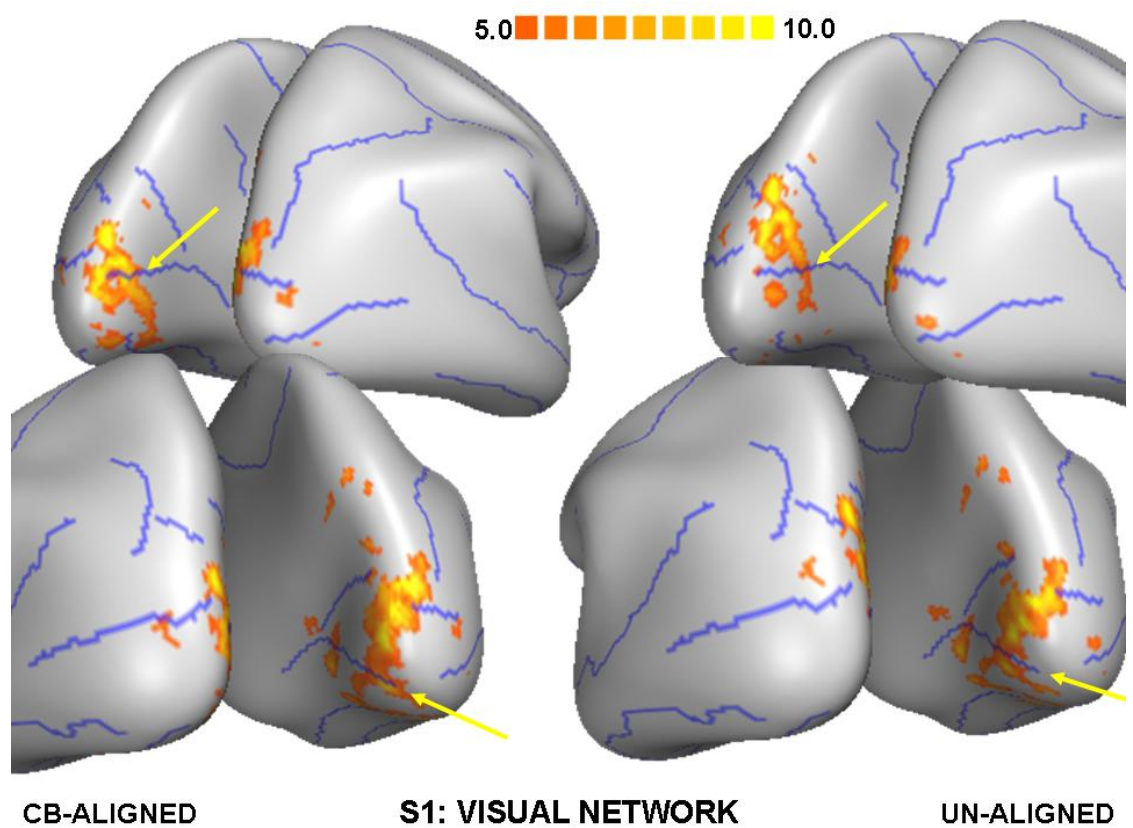

Supporting Information B. This figure demonstrates the difficulties of observing the effect of alignment on single subject Resting state data. Descriptively thresholded at $z=2$, overlaid on the average cortical mesh together with own individual (cb-aligned) major sulci, with and without cb-alignment applied to the map. In this figure, the change in the locations of the main spots is evident; however, the relation of the patterns to the anatomical borders and landmarks is less straightforward to interpret, as only small parts of the activity "increase" their respect the anatomical borders (e. g. a better covering of the bilateral calcarine sulci) with CBA applied compared to a non-aligned map. 


\section{References}

Allen, E.A., Erhardt, E.B., Damaraju, E., Gruner, W., Segall, J.M., Silva, R.F., Havlicek, M., Rachakonda, S., Fries, J., Kalyanam, R., Michael, A.M., Caprihan, A., Turner, J.A., Eichele, T., Adelsheim, S., Bryan, A.D., Bustillo, J., Clark, V.P., Feldstein Ewing, S.W., Filbey, F., Ford, C.C., Hutchison, K., Jung, R.E., Kiehl, K.A., Kodituwakku, P., Komesu, Y.M., Mayer, A.R., Pearlson, G.D., Phillips, J.P., Sadek, J.R., Stevens, M., Teuscher, U., Thoma, R.J., Calhoun, V.D. (2011). A baseline for the multivariate comparison of resting-state networks. Front Syst Neurosci 5, 2.

Argall, B.D., Saad, Z.S., Beauchamp, M.S. (2006). Simplified intersubject averaging on the cortical surface using SUMA. Hum Brain Mapp 27, 14-27.

Biswal, B., Yetkin, F.Z., Haughton, V.M., Hyde, J.S. (1995). Functional connectivity in the motor cortex of resting human brain using echo-planar MRI. Magn Reson Med 34, 537-541.

Belin, P., Zatorre, R.J., Ahad, P. (2002). Human temporal-lobe response to vocal sounds. Brain Res Cogn Brain Res 13, 17-26.

Binder, J.R., Frost, J.A., Hammeke, T.A., Bellgowan, P.S., Springer, J.A., Kaufman, J.N., Possing, E.T. (2000). Human temporal lobe activation by speech and nonspeech sounds. Cereb Cortex 10, 512-528.

Buckner, R.L., Andrews-Hanna, J.R., Schacter, D.L. (2008). The brain's default network: anatomy, function, and relevance to disease. Ann $N$ Y Acad Sci 1124, 1-38.

Corbetta, M. (1998). Frontoparietal cortical networks for directing attention and the eye to visual locations: identical, independent, or overlapping neural systems? Proc Natl Acad Sci U S A 95, 831-838.

Culham, J.C., Cavanagh, P., Kanwisher, N.G. (2001). Attention response functions: characterizing brain areas using fMRI activation during parametric variations of attentional load. Neuron 32, 737-745. 
Damoiseaux, J.S., Greicius, M.D. (2009). Greater than the sum of its parts: a review of studies combining structural connectivity and resting-state functional connectivity. Brain Struct Funct 213, 525-533.

Damoiseaux, J.S., Rombouts, S.A., Barkhof, F., Scheltens, P., Stam, C.J., Smith, S.M., Beckmann, C.F. (2006). Consistent resting-state networks across healthy subjects. Proc Natl Acad Sci U S A 103, 13848-13853.

De Luca, M., Smith, S., De Stefano, N., Federico, A., Matthews, P.M. (2005). Blood oxygenation level dependent contrast resting state networks are relevant to functional activity in the neocortical sensorimotor system. Exp Brain Res 167, 587-594.

Deco, G., Jirsa, V.K., McIntosh, A.R. (2011). Emerging concepts for the dynamical organization of resting-state activity in the brain. Nat Rev Neurosci $12,43-56$.

De Martino, F., Esposito, F., van de Moortele, P.F., Harel, N., Formisano, E., Goebel, R., Ugurbil, K., Yacoub, E. (2011). Whole brain high-resolution functional imaging at ultra high magnetic fields: an application to the analysis of resting state networks. Neuroimage 57, 1031-1044.

Esposito, F., Aragri, A., Piccoli, T., Tedeschi, G., Goebel, R., Di Salle, F. (2009). Distributed analysis of simultaneous EEG-fMRI time-series: modeling and interpretation issues. Magn Reson Imaging 27, 1120-1130.

Esposito, F., Goebel, R. (2011). Extracting functional networks with spatial independent component analysis: the role of dimensionality, reliability and aggregation scheme. Curr Opin Neurol 24, 378-385.

Esposito, F., Pignataro, G., Di Renzo, G., Spinali, A., Paccone, A., Tedeschi, G., Annunziato, L. (2010). Alcohol increases spontaneous BOLD signal fluctuations in the visual network. Neuroimage 53, 534-543.

Esposito, F., Scarabino, T., Hyvarinen, A., Himberg, J., Formisano, E., Comani, S., Tedeschi, G., Goebel, R., Seifritz, E., Di Salle, F. (2005). Independent component analysis of fMRI group studies by self-organizing clustering. Neuroimage 25, 193-205. 
Filippi, M., Valsasina, P., Misci, P., Falini, A., Comi, G., Rocca, M.A. (2012). The organization of intrinsic brain activity differs between genders: A resting-state fMRI study in a large cohort of young healthy subjects. Hum Brain Mapp Epub ahead of print.

Fischl, B., Sereno, M.I., Tootell, R.B., Dale, A.M. (1999). High-resolution intersubject averaging and a coordinate system for the cortical surface. Hum Brain Mapp 8, 272-284.

Fransson, P., Skiold, B., Horsch, S., Nordell, A., Blennow, M., Lagercrantz, H., Aden, U. (2007). Resting-state networks in the infant brain. Proc Natl Acad Sci U S A 104, 15531-15536.

Frost, M.A., Goebel, R. (2012). Measuring structural-functional correspondence: Spatial variability of specialised brain regions after macro-anatomical alignment. Neuroimage 59, 1369-1381.

Goebel, R., Esposito, F., Formisano, E. (2006). Analysis of functional image analysis contest (FIAC) data with brainvoyager QX: From single-subject to cortically aligned group general linear model analysis and self-organizing group independent component analysis. Hum Brain Mapp 27, 392-401.

Greicius, M.D., Supekar, K., Menon, V., Dougherty, R.F. (2009). Restingstate functional connectivity reflects structural connectivity in the default mode network. Cereb Cortex 19, 72-78.

Hyvarinen, A., Oja, E. (2000). Independent component analysis: algorithms and applications. Neural Netw 13, 411-430.

Kay, B.P., Meng, X., Difrancesco, M.W., Holland, S.K., Szaflarski, J.P. (2012). Moderating effects of music on resting state networks. Brain Res 1447, 53-64.

Kriegeskorte, N., Goebel, R. (2001). An efficient algorithm for topologically correct segmentation of the cortical sheet in anatomical mr volumes. Neuroimage 14, 329-346.

Luo, C., Guo, Z.W., Lai, Y.X., Liao, W., Liu, Q., Kendrick, K.M., Yao, D.Z., Li, H. (2012). Musical training induces functional plasticity in percep- 
tual and motor networks: insights from resting-state FMRI. PLoS One 7, e36568.

Mantini, D., Gerits, A., Nelissen, K., Durand, J.B., Joly, O., Simone, L., Sawamura, H., Wardak, C., Orban, G.A., Buckner, R.L., Vanduffel, W. (2011). Default mode of brain function in monkeys. J Neurosci 31, 1295412962.

Mantini, D., Perrucci, M.G., Del Gratta, C., Romani, G.L., Corbetta, M. (2007). Electrophysiological signatures of resting state networks in the human brain. Proc Natl Acad Sci U S A 104, 13170-13175.

Meindl, T., Teipel, S., Elmouden, R., Mueller, S., Koch, W., Dietrich, O., Coates, U., Reiser, M., Glaser, C. (2010). Test-retest reproducibility of the default-mode network in healthy individuals. Hum Brain Mapp 31, 237-246.

Mohammadi, B., Kollewe, K., Samii, A., Krampfl, K., Dengler, R., Munte, T.F. (2009). Changes of resting state brain networks in amyotrophic lateral sclerosis. Exp Neurol 217, 147-153.

Perrot, M., Riviere, D., Mangin, J.F. (2011). Cortical sulci recognition and spatial normalization. Med Image Anal 15, 529-550.

Rack-Gomer, A.L., Liau, J., Liu, T.T. (2009). Caffeine reduces resting-state BOLD functional connectivity in the motor cortex. Neuroimage 46, 56-63.

Raichle, M.E., MacLeod, A.M., Snyder, A.Z., Powers, W.J., Gusnard, D.A., Shulman, G.L. (2001). A default mode of brain function. Proc Natl Acad Sci U S A 98, 676-682.

Raichle, M.E., Snyder, A.Z. (2007). A default mode of brain function: a brief history of an evolving idea. Neuroimage 37, 1083-1090; discussion 1097-1089.

Rilling, J.K., Barks, S.K., Parr, L.A., Preuss, T.M., Faber, T.L., Pagnoni, G., Bremner, J.D., Votaw, J.R. (2007). A comparison of resting-state brain activity in humans and chimpanzees. Proc Natl Acad Sci U S A 104, 1714617151. 
Rombouts, S.A., Barkhof, F., Goekoop, R., Stam, C.J., Scheltens, P. (2005). Altered resting state networks in mild cognitive impairment and mild Alzheimer's disease: an fMRI study. Hum Brain Mapp 26, 231-239.

Rousseeuw, P.J. (1987). Silhouttes: a graphical aid to the interpretation and validation of a cluster analysis. Journal of Computational and Applied Mathematics 20, 53-65.

Senden, M., Goebel, R., Deco, G. (2012). Structural connectivity allows for multi-threading during rest: The structure of the cortex leads to efficient alternation between resting state exploratory behavior and default mode processing. Neuroimage 60, 2274-2284.

Smith, S.M., Fox, P.T., Miller, K.L., Glahn, D.C., Fox, P.M., Mackay, C.E., Filippini, N., Watkins, K.E., Toro, R., Laird, A.R., Beckmann, C.F. (2009). Correspondence of the brain's functional architecture during activation and rest. Proc Natl Acad Sci U S A 106, 13040-13045.

Takeuchi, H., Taki, Y., Hashizume, H., Sassa, Y., Nagase, T., Nouchi, R., Kawashima, R. (2012). The Association between Resting Functional Connectivity and Creativity. Cereb Cortex Epub ahead of print.

Talairach, J., Tournoux, P. (1980). Co-planar sterotaxic atlas of the human brain. Thieme, Stuttgart.

Thompson, P., Toga, A.W. (1996). A surface-based technique for warping three-dimensional images of the brain. IEEE Trans Med Imaging 15, 402417.

Vahdat, S., Darainy, M., Milner, T.E., Ostry, D.J. (2011). Functionally specific changes in resting-state sensorimotor networks after motor learning. $J$ Neurosci 31, 16907-16915.

van de Ven, V.G., Formisano, E., Prvulovic, D., Roeder, C.H., Linden, D.E. (2004). Functional connectivity as revealed by spatial independent component analysis of fMRI measurements during rest. Hum Brain Mapp 22, 165178.

van den Heuvel, M.P., Mandl, R.C., Kahn, R.S., Hulshoff Pol, H.E. (2009). Functionally linked resting-state networks reflect the underlying structural 
connectivity architecture of the human brain. Hum Brain Mapp 30, 31273141 . 


\section{CHAPTER 4}

\section{ONE OR TWO ANALYSES? AN INTEGRATED APPROACH FOR WHOLE-CORTEX MACRO- ANATOMICAL AND ROI-BASED FUNCTIONAL}

ALIGNMENT

Corresponding publication:

Frost, M.A. and Goebel, R. (2012) One or Two Analyses? An Integrated Approach for Whole-Cortex Macro-Anatomical and ROI-Based Functional Alignment. Submitted. 


\begin{abstract}
In this paper we present a new alignment procedure which incorporates anatomical and functional information from each subject. In this way we were able to create a group cortical reconstruction which is well aligned in terms of both anatomy and function. Due to the anatomical variability across subjects, many brain mapping experiments choose to focus their analysis on one particular region of interest so as to circumvent the problem of sub-optimal statistics resulting from the lack of anatomical correspondence. This means that two analysis need to be carried out, one on the region of interest alone, and a separate 'whole brain' analysis. Using this alignment scheme which integrates anatomical and functional information, we propose that two analyses may not be necessary. After an integrated structural and functional alignment one is able to carry out a whole brain analysis in the confidence that not statistical power is lost due to spatial variation in the location of functional regions of interest.
\end{abstract}




\section{Introduction}

The healthy human brain is generally comprised of a typical folding pattern which is more or less consistent across our species, e.g. the central sulcus runs in a vertical/horizontal axis near the middle of the lateral cortical surface and the calcarine sulcus runs along the medial surface of the occipital pole. This folding pattern (macroanatomy) specifies the location of "functional areas" in a remarkably consistent way across the population, e.g., the primary visual cortex, V1, can be found around the calcarine sulcus and shares a border with the secondary visual cortex, V2 (Sereno et al., 1995) and the frontal eye field can be found in the intersection of the superior frontal and precentral sulcus (Paus, 1996). Despite these inherent similarities no two brains are exactly the same. The small, but significant differences in structure and function in the brain are surely part of what makes us individuals, but at the same time this variability contributes to a decrease in statistical power of any multi-subject whole-brain fMRI analysis. The present paper attempts to address this issue by describing an integrated alignment approach that not only minimises the macroanatomical variability across subjects but is also informed by homologue functional data to drive alignment. We show that this approach results in a group aligned space with strong spatial correspondence of both structure and function while maintaining maximal anatomical fidelity.

For whole-brain group studies, anatomical and functional images of each subject are usually normalised to a standard 3D space with the MNI (Evans et al., 1992) and Talairach (Talairach and Tournoux, 1980) transformations being most commonly used. By normalising images to these standard templates one is able to account for gross differences in brain size and orientation through piecewise linear (Talairach) or affine (MNI) transformations. These normalisation techniques however are blind to the idiosyncrasies of an individual's cortical folding pattern. Consequently, although the brains may be in a standard space allowing for comparisons to be made across a group, these comparisons are not necessarily drawn from homologous anatomical (or functional) areas across subjects (Van Essen and Drury, 1997). The widespread use of referring to co-ordinate locations derived from these standard spaces to communicate foci of activation, while in practice is very useful, must be treated cautiously as any given co-ordinate may correspond to a different area in individual subjects. 
A number of advanced alignment techniques exist which are capable of bringing individual brains into better correspondence. Some volume based approaches utilise intensity values of the images to improve inter-subject registration (Amunts and Zilles, 2001; Ashburner, 2007; see (Klein et al., 2009) for a comparison of volume based approaches). For macroanatomical alignment, a more promising approach is to use the information from reconstructed cortical surface meshes, as opposed to the volume data, to drive alignment. Both manually labelled landmark alignment (Van Essen, 2005) and curvature driven alignment (extracted automatically from the folding pattern of a cortical surface reconstruction) have been shown to be powerful surface-based tools to maximise cross-subject alignment of macro-anatomy. These surface based tools are readily available in analysis software packages (Brain Voyager, Caret, Freesurfer).

We recently demonstrated that, after advanced curvature driven cortex based alignment (CBA), macroantomical variability between subjects is substantially reduced and indeed almost completely removed for major sulci. Furthermore as a consequence of increasing correspondence of anatomy we observed a concomitant increase in the overlap of a set of investigated functional areas across subjects (Frost and Goebel, 2012). The gain in overlap after cortex based alignment was not uniform across all function areas, suggesting that some functional areas are strongly related to macro-anatomy e.g. the frontal eye fields (Paus, 1996) whereas others are not as tightly bound e.g. the fusiform face area (FFA, but see Weiner \& Grillspector (2010) and discussion in Frost \& Goebel (2012)).

In order to overcome the problem that there remain differences in the spatial location of functional areas after registration to a standard template, and even after advanced alignment schemes, a region of interest (ROI) approach is often utilised. Restricting analysis to a few circumscribed, independently localised regions allows to carry out group comparisons of functionally homologous regions across subjects with high statistical power and substantially reduces the correction needed for multiple comparisons as is needed for the many thousands of voxels of a whole brain analysis. However this approach ignores activity in areas outside the ROIs which would be revealed by a whole-brain analysis. While one could perform both kinds of analyses separately (as is indeed reported in many published studies), an integrated approach combining ROI and whole brain analysis with maximal correspondence across subjects would be desirable. Indeed there has been a debate on 
the usefulness and necessity of an independent scan to define a ROI (Friston and Henson, 2006; Friston et al., 2006; Saxe et al., 2006). The argument, put forth by Friston et al. (2006) was that independent functional localising scans, used for the sole purpose of selecting a ROI, were not necessary for most of the questions posed by neuroimaging experiments if a balanced, fully factorial design was implemented. This proposition was questioned by Saxe et al. (2006) who explicitly raised the concern that, due to anatomical variability between subjects, coupled with the fact that not all functional areas respect macro-anatomical landmarks and normalizing to a standard template does not resolve these problems, the most practical solution is to focus one's analysis to a functionally specified region, defined by a localising scan, independent from the main experiment.

In light of this debate, we propose an integrated alignment technique which incorporates the spatial specificity and statistical power of a region of interest analysis, with the benefits of a whole brain analysis. To indicate that the whole-cortex alignment incorporates functional information from localised ROIs, we refer to the developed procedure as functionally informed cortex based alignment (fCBA). Due to the fact that a purely macro-anatomical alignment is not capable of successfully bringing functional regions fully into spatial correspondence, fCBA integrates both curvature (anatomical information) and functionally defined regions to produce a group brain, which is well aligned in terms of anatomy and function. By carrying out a group alignment that is informed by both anatomical and functional information from each subject, we argue that two separate analyses (ROI based and whole-brain) are no longer required for the analysis of subsequent main experiments. Instead, having overcome the structural-functional differences across subjects through this alignment procedure, one is free to perform a single whole-brain analysis, with the power of specificity for localised regions that was previously only obtained in a ROI based analysis.

There has been previous research which included functional information to improve spatial correspondence across subjects. Sabuncu et al (2010) performed a multi-subject alignment primarily based on the functional activity measured while watching half a feature length film with the interest being on aligning patterns of activation. Our approach differs in that we aim to align discrete functionally defined areas that are often defined for a ROI analysis and can be localised in just a few minutes of scanning time. To demonstrate our integrated approach, we use functional localisers for three selected 
prominent areas, FFA, hMT+ and FEF, and use these defined regions to inform multi-subject cortex alignment. We expect that, by increasing spatial correspondence of functional, as well as anatomical regions across subjects, an improvement in group statistics will be achieved over purely (macro-) anatomical alignment.

\section{Materials and Methods}

\subsection{Participants}

10 subjects ( 4 female, 6 male) with a mean age of 31.3 (range 25-46) were scanned as part of a larger project (Frost and Goebel, 2012).

\subsection{Anatomical Alignment}

All anatomical volumes and functional time series were first warped into Talairach space. This operation included the definition of the landmarks AC (anterior commissure), PC (posterior commissure) and the borders of the cerebrum; the defined subject-specific landmarks were then used to rotate each brain in the AC-PC plane followed by piecewise, linear transformations to fit each brain in the common Talairach "proportional grid" system (Talairach and Tournoux, 1980). Then an automatic segmentation procedure was applied leading to the creation of topologically correct cortical surface reconstructions (Kriegeskorte and Goebel, 2001). Mean curvature values were then obtained from these reconstructed cortical meshes. These curvature maps are used to drive alignment of residual macro-anatomical variability. Firstly through a stage of rigid alignment followed by non-rigid warping which proceeds in a coarse to fine fashion with smoothed curvature maps aligning the most prominent anatomical features (e.g. Sylvian fissure, central sulcus, intraparietal sulcus, calcarine sulcus) for a certain number of iterations before proceeding to less smoothed curvature maps which include finer details. This procedure continues until the least smooth, most detailed, curvature maps have been aligned across subjects. CBA utilises a moving target approach where alignment is driven by a dynamic group average curvature map that itself reflects the progress of macro-anatomical alignment (for details see Goebel et al., 2006). 


\subsection{Functional Localisers}

The fusiform face area (FFA) was localised using an established paradigm, which consisted of blocks of faces, houses, objects and scrambled objects presented for 16 seconds in a random order with every alternate block consisting of a rest period of central fixation. Each single stimulus was presented for $666 \mathrm{~ms}$ with 17 milliseconds fixation in between each image. In all block types subjects were instructed to keep central fixation. The contrast used to localise FFA was "faces > houses" and for PPA it was "houses > faces".

To localise $\mathrm{V} 5 / \mathrm{hMT}+$, an area sensitive to visual motion, a paradigm was used with two main conditions showing either 400 dots moving radially outwards from the centre of the screen or showing 400 stationary dots (Goebel et al., 1998). Four blocks of each experimental condition were presented to the subject and each alternate block contained a central fixation point and served as a block of rest. Subjects were instructed to keep central fixation during all block types. Area V5/hMT+ was identified using the contrast "moving dots > stationary dots".

The frontal eye fields (FEF) were localised using a saccadic eye movement task. Subjects were instructed to make eye movements to follow a dot which would appear in one of 8 locations on the vertical and horizontal meridians at a rate of $2 \mathrm{~Hz}$ for 16 seconds. Each task block was followed by a block of equal length where subjects were asked to fixate a cross displayed in the centre of the screen. The contrast used to label FEF was "saccades > fixation".

\section{4 fMRI scanning parameters}

All subjects were scanned with a Siemens 3T head only scanner (Magnetom Allegra, Siemens Medical Systems, Erlangen, Germany). A standard echoplanar-imaging (EPI) sequence (volume repetition time $[\mathrm{TR}]=2$ secs, field of view $[\mathrm{FOV}]=224 \mathrm{~mm} \times 224 \mathrm{~mm}$, matrix size $=64 \times 64$, echo time $[\mathrm{TE}]=30 \mathrm{~ms}$ ) with a voxel size of $3.5 \mathrm{~mm}^{3}$. Each volume consisted of 32 slices, covering the whole brain. A high-resolution structural scan (voxel size, $1 \times 1 \times 1 \mathrm{~mm}^{3}$ ) was also collected for each subject (in each session) using a T1-weighted three-dimensional (3D) ADNI sequence [TR, $2050 \mathrm{~ms}$; echo time (TE), $2.6 \mathrm{~ms}$; 192 sagittal slices]. 


\subsection{Integrated structural and functional $C B A(f C B A)$}

All data from the three functional localising paradigms were cut in half resulting in two splits with an equal number of time points per split ( $83 \mathrm{vol}-$ umes for the FEF localiser, 195 for the FFA localiser and 50 for the hMT+ localiser). The respective time course data were used to label the three functional regions on each of the subjects' own cortical mesh reconstruction, to be used along with curvature information, to drive inter-subject alignment. No further alignment (beyond Talairach normalisation) was carried out in volume space. For the sole purpose of alignment, the functionally labelled areas were smoothed with a Gaussian kernel so as to provide a gradient into the area's neighbourhood to be followed during the alignment procedure maximising overlap across subjects. The integrated alignment process followed the stages of CBA as previously described and included the coarse-tofine alignment of curvature information to a dynamic group average.

Table I.

\begin{tabular}{|c|c|c|c|c|c|c|}
\hline & \multicolumn{3}{|c|}{ fCBA I } & \multicolumn{3}{c|}{ fCBA II } \\
\hline Order & $\begin{array}{c}\text { Functional } \\
\text { Weighting }\end{array}$ & $\begin{array}{c}\text { Number of } \\
\text { updates }\end{array}$ & $\begin{array}{c}\text { Number of } \\
\text { iterations }\end{array}$ & $\begin{array}{c}\text { Functional } \\
\text { Weighting }\end{array}$ & $\begin{array}{c}\text { Number of } \\
\text { updates }\end{array}$ & $\begin{array}{c}\text { Number of } \\
\text { iterations }\end{array}$ \\
\hline 1 & 0.2 & 4 & 400 & 0.2 & 4 & 400 \\
\hline 2 & 0.4 & 4 & 300 & 0.4 & 4 & 300 \\
\hline 3 & 0.6 & 3 & 300 & 0.6 & 4 & 300 \\
\hline 4 & 0.9 & 10 & 1000 & 0.8 & 4 & 300 \\
\hline 5 & 0.2 & 4 & 400 & 0.9 & 4 & 400 \\
\hline
\end{tabular}

Table I. Details of the parameters of the two fCBA procedures at different phases using strongly smoothed (order 1) to minimally smoothed (order 4 and 5) curvature maps. The 'functional weighting' column indicates the weighting of functional information wf, relative to anatomical weighting wa $(\mathrm{wa}=1.0-\mathrm{wf})$ during the two fCBA alignment procedures. fCBA I progressively increased the weighting to functional information but concluded with a strong weighting of curvature information. fCBA II progressively increased weighting to functional information. 'Number of updates' indicates how many times the group average 
was updated as the dynamic target of alignment and 'number of iterations' how many iterations were performed in each alignment phase.

In addition to the anatomical alignment, the fCBA procedure allowed to specify a weighting between functionally driven and curvature driven alignment, which was preset to vary at different intervals over the course of alignment with decreasing smoothness of curvature maps (rows in table 1). Using the weighting of functional information $\mathrm{w}_{\mathrm{f}}$ relative to anatomical weighting $\mathrm{w}_{\mathrm{a}}$ with $\mathrm{w}_{\mathrm{f}}=1.0-\mathrm{w}_{\mathrm{a}}$, two different fCBA procedures were carried out, here called fCBA I and fCBA II. The first procedure used a progressively stronger weighting to functional information but added a final step with a high weighting of macroanatomical (curvature) information with the goal to quickly realign fine-grained macroanatomy without loosing achieved functional alignment. This strategy was the result of observations showing a decline in macroanatomical alignment when using strong forces for functional alignment over an extended period of time. The second fCBA procedure continued to increase the weighting of the alignment in favour of the functional regions, i.e. it optimised the alignment of the functionally defined regions tolerating reduction in macroanatomical alignment (see table I).

In order to compare surface based alignment with normalised volume space the surface labelled functional areas were back-projected from each individual's reconstructed cortex mesh to Talairach normalised volume space.

\subsection{Analysis}

All Analysis was carried out using BrainVoyager QX 2.6 software package (Brain Innovation, Maastricht, The Netherlands).

Two measures were used to test the efficacy of functionally driven alignment. Firstly functional regions were defined based on the second half of the split data of the three localiser experiments and aligned using the output of the standard CBA and the two fCBA procedures. Alignment was assessed by calculating across-subject overlap $o$ per mesh vertex $v$ was using the formula $o_{v}=N_{v} / N$ with $N_{v}$ indicating the number of subjects that have the same localised region at vertex $v$ and $N$ indicating the total number of subjects $(N=$ $10)$. 
Secondly, the effect on group-level statistics was evaluated by carrying out a multi-subject general linear model analysis with the data co-registered following the different alignment schemes. A group-level region of interest was created for FFA, FEF and hMT+ from the union of areas of all 10 subjects defined for the overlap measure above from the second split of the data (i.e. that which was not used for alignment). The t-value of the 50 most active vertices were averaged to give a accurate sample of the effect of alignment on group statistics.

The effect of the three alignment schemes on macro-anatomical correspondence was assessed in order to reveal how much macroanatomical alignment "suffers" when functional alignment forces are included. Macro-anatomical correspondence was quantified by demarcating the fundus of 26 sulci and measuring the inter-subject Hausdorff distance of these landmarks (Frost and Goebel, 2012; Pantazis et al., 2010).

\section{Results}

By integrating both curvature and functional information in our multi-subject alignment scheme we observed increases in the overlap of an independently defined (not used to drive alignment) data set. Figure 1 shows the gains in overlap across alignment schemes. As expected from previous work, the lowest amount of overlap was observed when functional regions were projected into the volume that had been normalised to the Talairach space. Overlap across subjects increased when functional regions were projected onto the surface, even before any alignment was applied. After the purely anatomical CBA procedure a further gain in overlap was observed across all functional areas. The inclusion of functional areas in the subsequent two procedures (fCBA I and II) increased the overlap of independently defined functional areas further with the largest overlap being observed after the alignment with the strongest weighting given to functional information.

Figure 1 displays the effect of the different alignment schemes on the underlying anatomy. There is a high degree of variability (distance between subjects' anatomical sulcal paths) without explicit cortex-based alignment. The application of anatomical CBA greatly reduces this variability. When functional information was included in the alignment scheme the distance between subjects' anatomical landmarks was only slightly increased with re- 
spect to $\mathrm{CBA}$ alone. We also observed more reduction of anatomical alignment for fCBA II than fCBA I.

TABLE II.

\begin{tabular}{|c|c|c|c|c|}
\hline & Native Surface & CBA & fCBA I & fCBA II \\
\hline \multicolumn{5}{|l|}{ Left Hemisphere } \\
\hline Central Sulcus & 9.1788 & 0.08282 & 0.075723 & 0.255035 \\
\hline Precentral Sulcus & 10.7181 & 0.61689 & 1.12725 & 1.6933 \\
\hline Cingulate Sulcus & 3.6437 & 0.81756 & 0.935932 & 1.56864 \\
\hline Collateral Sulcus & 7.2826 & 1.61858 & 2.11129 & 2.79816 \\
\hline Occipito Parietal Sulcus & 22.6545 & 0.87846 & 1.60967 & 5.50819 \\
\hline Calcarine Sulcus & 10.2366 & 0.2176 & 0.366921 & 5.0058 \\
\hline Average & 10.61905 & 0.705318 & 1.037798 & 2.804854 \\
\hline \multicolumn{5}{|l|}{ Right Hemisphere } \\
\hline Central Sulcus & 6.22911 & 0.101734 & 0.19147 & 0.690856 \\
\hline Precentral Sulcus & 6.37196 & 2.10002 & 2.33101 & 3.77453 \\
\hline Cingulate Sulcus & 3.69301 & 1.51784 & 1.8313 & 2.73913 \\
\hline Collateral Sulcus & 12.6199 & 1.60612 & 2.44967 & 3.68469 \\
\hline Occipito Parietal Sulcus & 4.36663 & 0.491205 & 0.552402 & 1.9479 \\
\hline Calcarine Sulcus & 3.96938 & 1.00655 & 1.05801 & 1.52155 \\
\hline Average & 6.2083 & 1.1372 & 1.4023 & 2.3931 \\
\hline
\end{tabular}

Table II. Displays the variance of distance (mm2) of macro-anatomical (sulcal) landmarks between subjects. CBA substantially minimises the anatomical variability across subjects observed in original group space. Including functional regions in the multi-subject alignment procedure (fCBA I and fCBA II) increases the variance of anatomical landmarks but only slightly. 
Table II gives details of the 7 landmarks which were examined across alignment schemes and reveals that all 7 sulci showed the same pattern of variability across different alignment schemes. Every sulcal landmark was most variable in terms of distance between subjects in native, unaligned (Talairach normalised) surface space. This variability was greatly reduced following CBA. Overall fCBA I contributed to a very small increase in distance between subjects. fCBA II, with its strong weighting of functional information to guide alignment resulted in a further increase in the distance of anatomical landmarks between subjects. Importantly however the distance between subjects' sulci was still far smaller after fCBA II than it was in native space. The visible effect of these distortions can be seen in figures 3, 4 and 5. The anatomy appears best after CBA. The non aligned "identity" surface as well as fCBA II surface lack fine-grained anatomical details.

As well as an increase in overlap of these functionally defined regions we have also observed a gain in the power of the group statistics over the alignment schemes (see figure 2) as measured by the average t-values of the 50 most active vertices in the "group labelled" region comprised of the union of each individual's defined region, for each alignment scheme. As with the overlap measures, in all cases the group statistics improved with the addition of functional information in the alignment. The smallest group statistic power was observed when multi-subject GLM was carried out on the surface with no additional alignment besides a normalisation to Talairach space (Identity). The strongest group statistics results were observed after fCBA II in all areas. A multivariate ANOVA test revealed an effect of alignment scheme $[F(4)=16.63, p<0.05)]$. Post hoc t-tests revealed significant gains in statistical power when comparing CBA to simple Talairach surface space $(p<0.05)$. The group statistical effect observed after fCBA I was also significantly greater than after CBA $(\mathrm{p}<0.05)$ and despite the small difference in effect size between fCBA I and fCBA II there was a significant improvement in the t-statistic after the highly functional weighted fCBA II procedure $(\mathrm{p}<0.05)$. The differences in statistical power across alignment schemes is also visible on statistical surface maps (see figures 3 for the maps of FFA), figure 4 for maps of hMT+ and figure 5 for maps of FEF). 

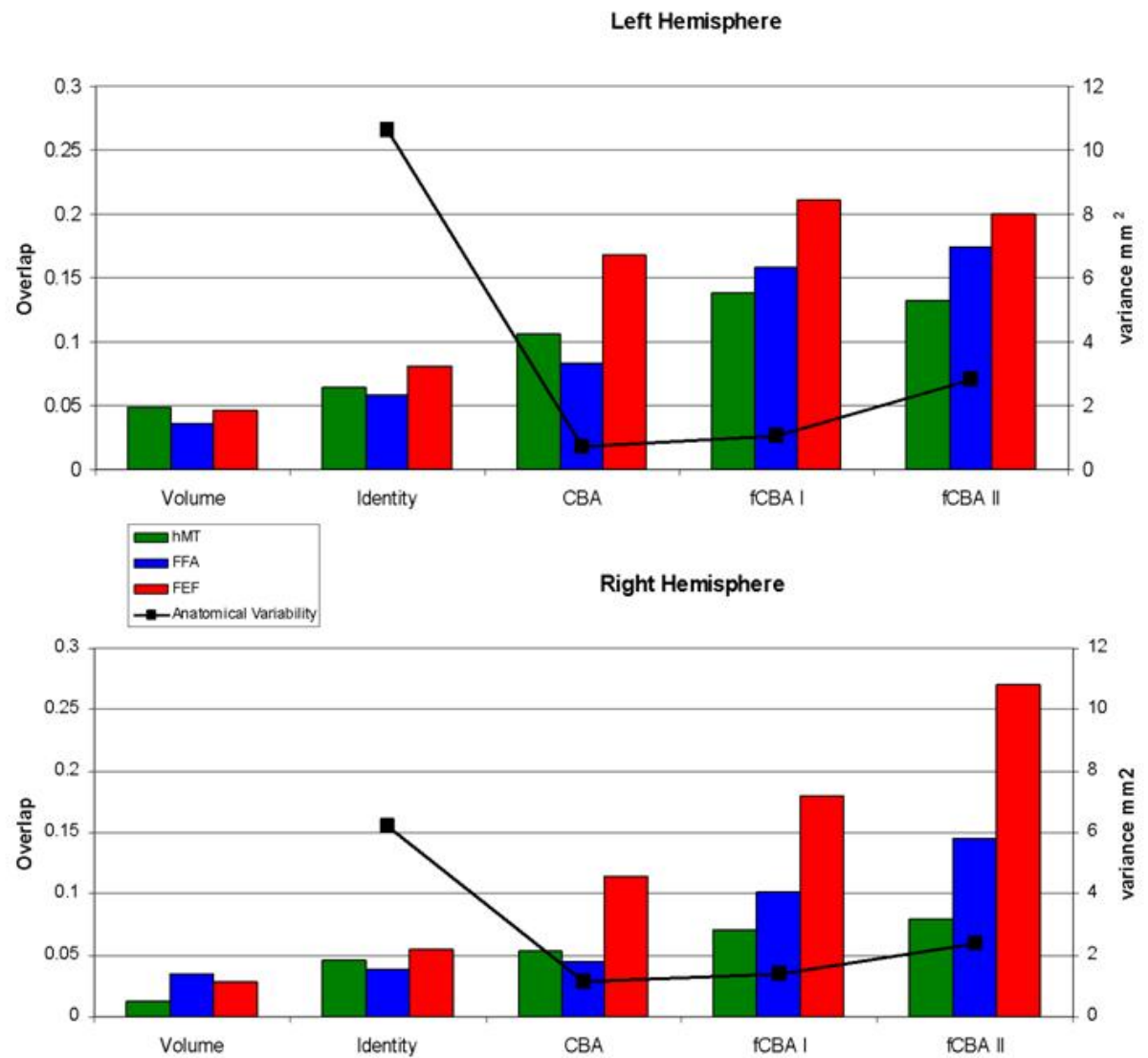

Figure 1. Bar graphs depicting the amount of overlap of functional areas across alignment schemes. Overlap increases with the addition of functional regions as targets of alignment (fCBA I, fCBAII) in addition to anatomical (curvature) information. The secondary y axis on the right displays the variance of 6 major sulci across alignment schemes (see table 2). CBA reduced the inherent macro-anatomical differences across subjects most strongly. fCBA does, however, not substantially increase the variance of anatomy. 
Differences in group statistics across alignment schemes LH

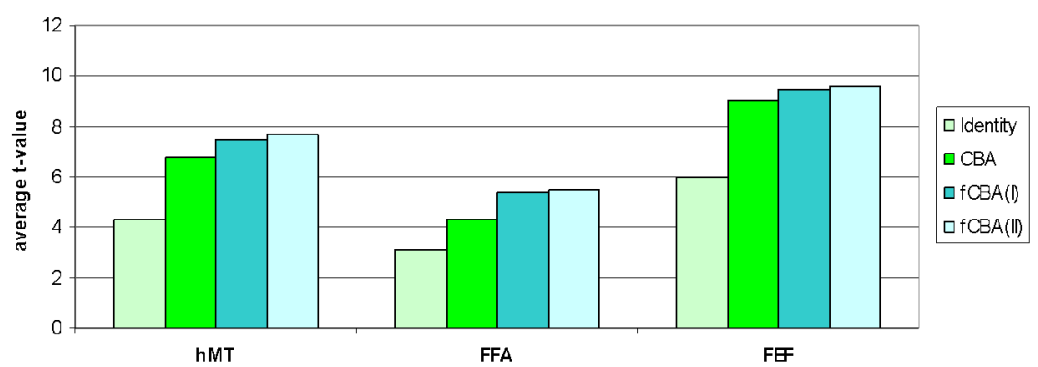

Diffe rences in group statistics across alignment schemes RH

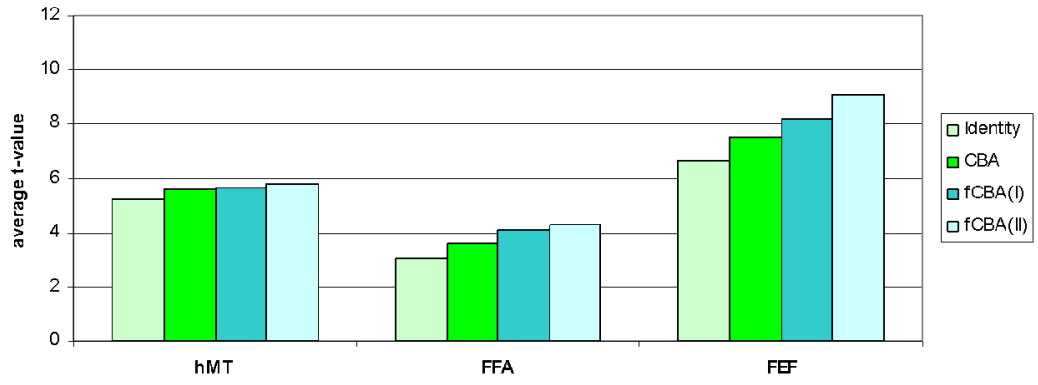

Figure 2. Bar graphs showing the average t-statistic values of the 50 most active voxels of each functional region after a group level GLM. These values are plotted for the 3 different functional regions and show that a pure anatomical alignment improves group statistics, yet further gains can be made using functional information to guide group alignment.

\section{Discussion}

With the addition of functional information into the group alignment procedure we were able to create a group average cortical space, which is well aligned both in terms of anatomy and function for pre-localised specialised areas. Our tests, carried out on an independent data set (i.e. not used to drive alignment), demonstrated that fCBA successfully aligned functional regions, as measured by an increase in the overlap of functionally labelled ROIs. Having brought these functional regions into alignment we also observed substantial increases in group statistical power thereby supporting our original hypothesis.

The findings presented here are particularly interesting in light of the earlier debate about the use of ROI analysis (Friston and Henson, 2006; Friston et 
al., 2006; Saxe et al., 2006). All parties involved in the debate agreed that the structural-functional differences which exist across subjects are an issue which needs to be addressed in neuroimaging as standard normalisation schemes are not capable of resolving these differences. Saxe et al. stated that, a ROI based analysis is a simple and effective solution in order to overcome the problem. Friston et al. posited that averaging the time-course of a ROI is a sub-optimal solution. We hope the approach outlined in this paper provides a new solution to this important issue. 


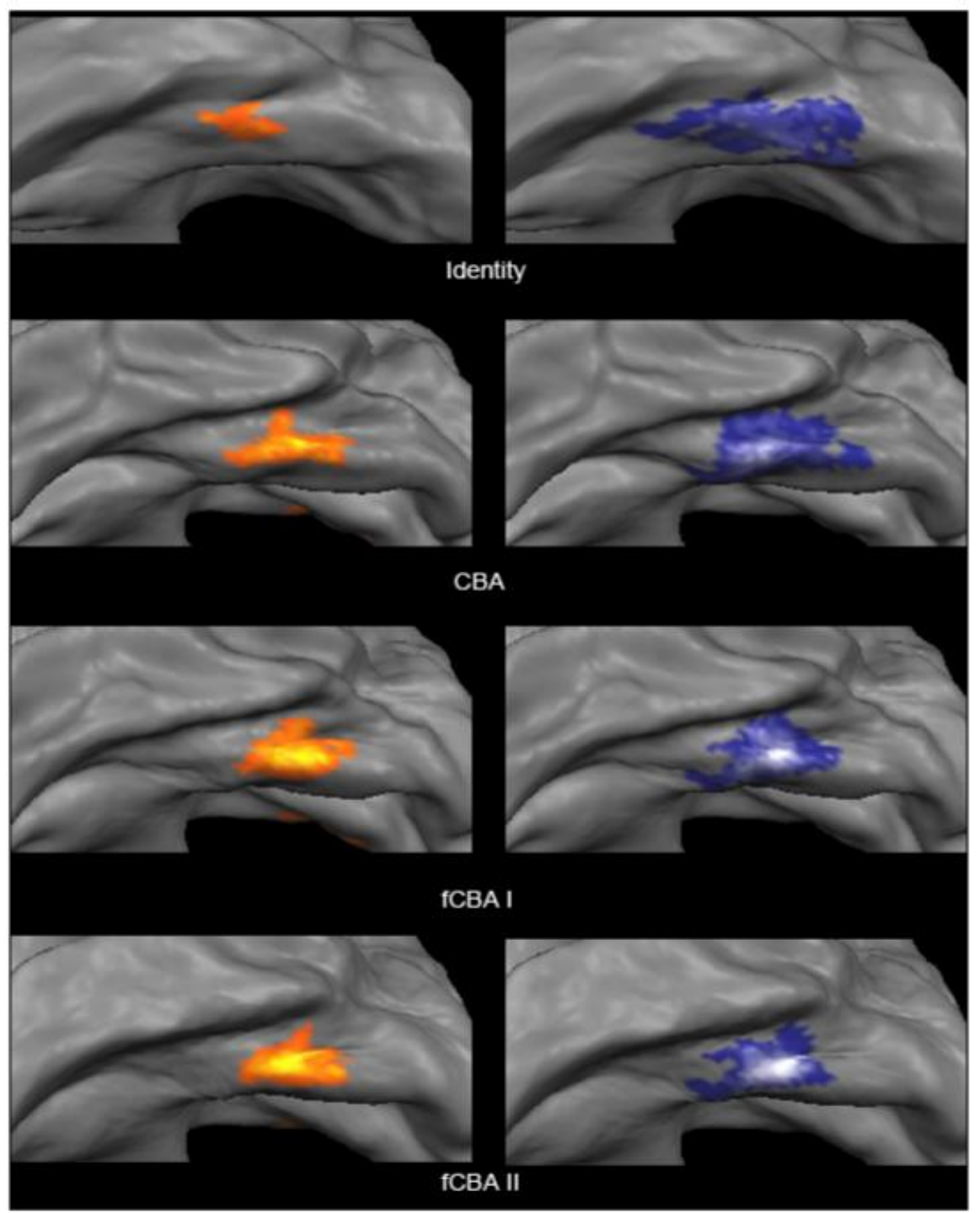

Figure 3. Group maps showing overlap of FFA (right column) as well as statistical maps of multi-subject RFX GLM of FFA (left column) from the second half of the data that was not used for alignment. On the left a brighter orange, approaching yellow, indicates stronger statistical $t$ values. The threshold is held constant across all three images depicting FFA with four alignment schemes. On the right a brighter blue, approaching white, indicates higher overlap of FFA (white = all subjects overlap). In the top row are the group maps with no surface based alignment. Directly below are the group maps after curvature driven CBA. Below that the group maps after fCBA I and finally on the bottom fCBA II with the strongest weighting given to functional data. The underlying anatomical group cortex meshes reflect the macro-anatomical alignment quality as obtained from the different alignment procedures. 


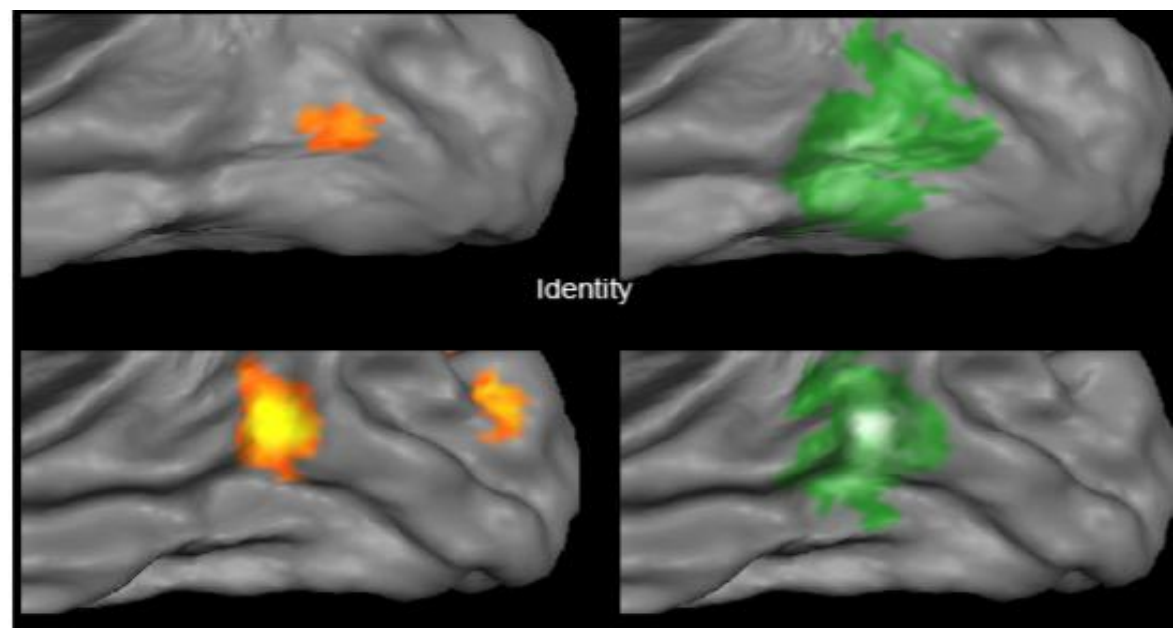

CBA
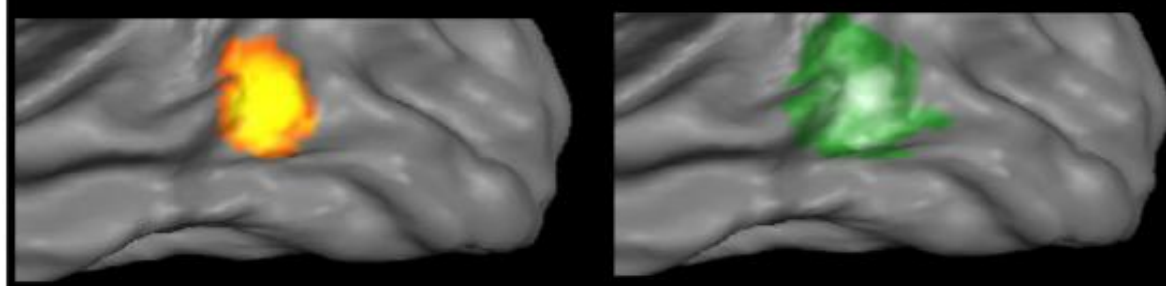

\section{fCBAI}

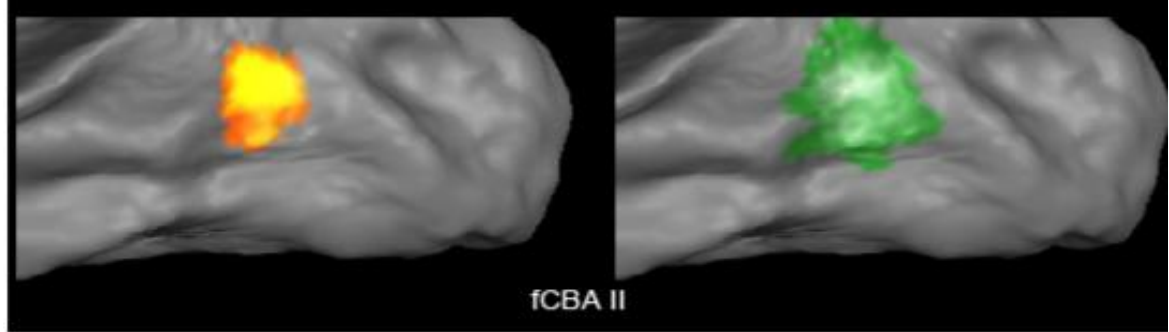

Figure 4. Group maps showing overlap of hMT+ (right column) as well as statistical maps of multi-subject RFX GLM of hMT+ (left column) from the second half of the data. i.e. not used for alignment. On the left a brighter orange, approaching yellow, indicates stronger statistical $\mathrm{t}$ values. The threshold is held constant across all three images depicting hMT + with four alignment schemes. On the right a brighter green, approaching white, indicates higher overlap of hMT+. In the top row are the group maps with no surface based alignment. Directly below are the group maps after curvature driven CBA. Below that the group maps after fCBA I and finally on the bottom fCBA II with the strongest weighting given to functional data. The underlying anatomical group cortex meshes reflect the macro-anatomical alignment quality as obtained from the different alignment procedures. 


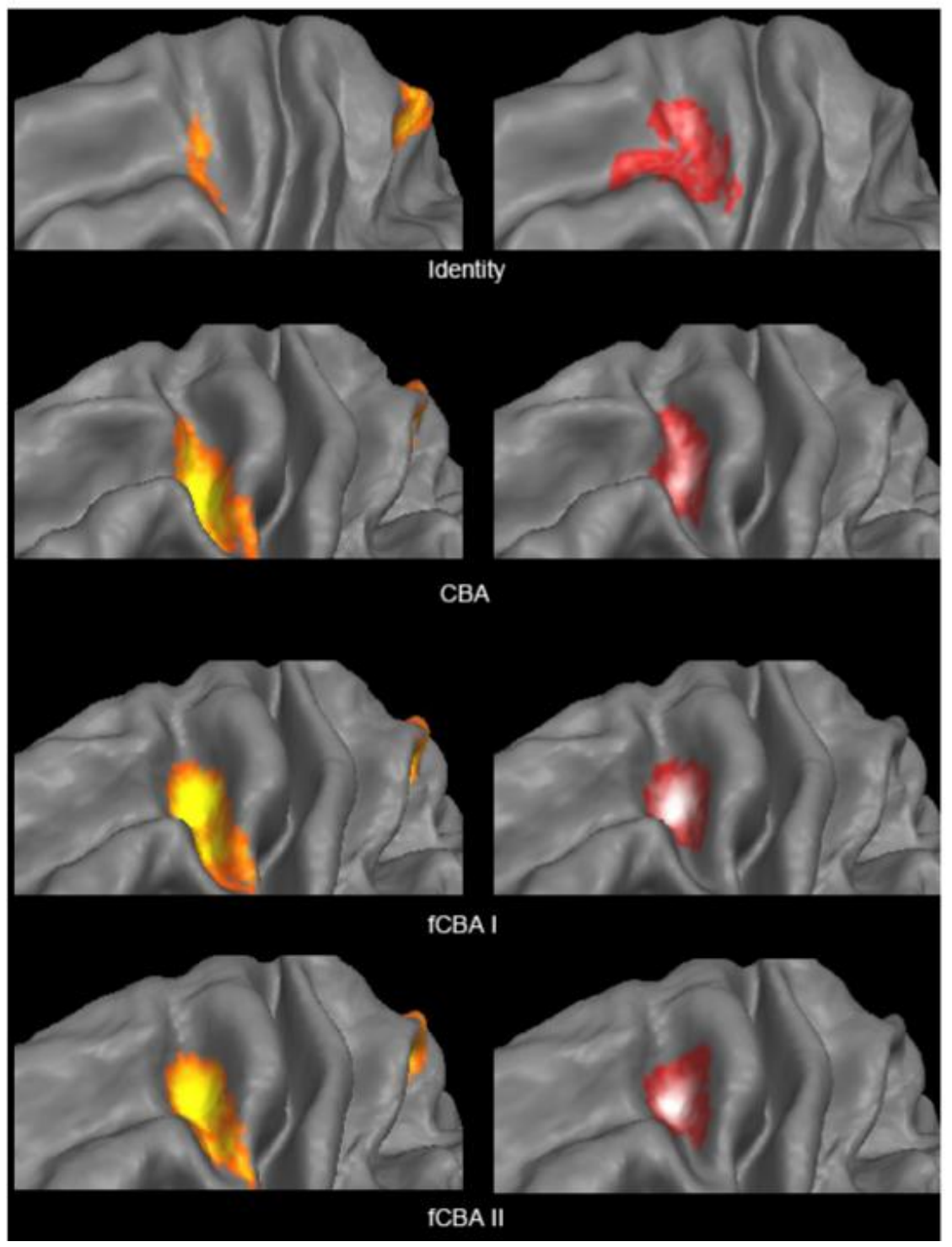

Figure 5. Group maps showing overlap of FEF (right column) as well as statistical maps of multi-subject RFX GLM of FEF (left column) from the second half of the data. i.e. not used for alignment. On the left a brighter orange, approaching yellow, indicates stronger statistical $\mathrm{t}$ values. The threshold is held constant across all three images depicting FEF with four alignment schemes. On the right a brighter red, approaching white, indicates higher overlap of FEF. In the top row are the group maps with no surface based alignment. Directly below are group maps after anatomical CBA. Below that the group maps after fCBA I and finally on the bottom fCBA II with the strongest weighting given to functional data. The underlying ana- 
tomical group cortex meshes reflect the macro-anatomical alignment quality as obtained from the different alignment procedures.

The new approach which we present here, functionally informed cortexbased alignment (fCBA), offers a way to integrate two analyses (ROI and whole-cortex) and obtain the benefits of both kinds with minimal additional time required for scanning localising experiments as is usually performed in many labs. Having aligned the brains of subjects based on structural and prelocalised functional data, one is able to proceed with a whole brain analysis, without the need to extract time courses from a small sample of the measured cortex, nor average a signal from a discrete area in order "to summarise regional responses" (Friston et al., 2006), thereby avoiding the negative aspects of a ROI analysis. At the same time, having implemented fCBA one would not sacrifice much statistical power due to spatial mis-match, thereby avoiding this disadvantage of whole-brain analysis.

Integrating functional regions in the group alignment process did not introduce excessive amounts of variability in the anatomy of the subjects. As expected, fCBA II resulted in a larger distance between subject's anatomical landmarks than CBA and fCBA I. It was surprising how little distortion fCBA I introduced to the underlying anatomy. In fact, despite the overall substantial gain in both overlap, and group statistical power, fCBA I resulted in almost no appreciable increase in variability of these sulcal paths, despite the fact that they were in close proximity to the functional areas brought into alignment. Two factors are likely responsible for this. Firstly fCBA I implemented high weightings for functional information during the alignment procedure, yet concluded with a cycle of iterations where the force of functional alignment was relaxed and anatomical information was the major driving force behind the alignment. It seems likely that this final stage of anatomical alignment successfully brought the anatomical landmarks back into optimal spatial correspondence. The other factor contributing to the minimal violation of anatomical correspondence of fCBA I is that the direction of alignment force of curvature is strongest orthogonal to the axis of the curvature being aligned whereas the alignment forces for functional regions do not prefer any particular axis. E.g., for the central sulcus, which runs along a superior/inferior axis, the macro-anatomical alignment is strongest in an anterior/posterior direction, where as for the collateral sulcus in the inferior temporal lobe this is in a medial/lateral direction as this sulcus lies in a direction along the anterior/posterior axis. The addition of functional regions as 
targets of alignment, if they are mitigated by addition iterations of curvature (anatomical) alignment, will not force these regions apart to a significant extent.

It must be noted that, having included functional information in the group alignment process, one cannot draw inferences about the fine-grained relationship between structure and function as the naturally occurring macroanatomical correspondence is altered during the fCBA process. For this reason alignment based solely on macro-anatomy must be used to address questions about structural-functional correspondence as described in Frost and Goebel (2012).

Due to the fact that we split the data in order to have one data set to base our functional alignments on, and a second independent data set to test the efficacy of the alignment, two sources of spatial variability were present in this study. Firstly the inter-subject variability which is present in every multisubject experiment, even after macro-anatomical alignment and secondly intra-subject variability, that is, the variation in spatial location of a functionally defined area within a subject, across two (or more) different measurements. Intra-subject variability has previously been shown to be almost inconsequential at a normal resolution $(3 \times 3 \times 3 \mathrm{~mm})$ as the spatial location of functional areas seems to be stable across scanning sessions (Peelen and Downing, 2005) as long as certain requirements are met, such as not too strict thresholding levels (Duncan et al., 2009). Indeed we observed very little variability in the location of the functional region, no matter whether they were labelled from the first or second split of the data set. Having said that, due to the fact that we were using half the data points usually used to localise these areas, one could expect even more accurate labelling when a full data set is used for localisation. In this way the gains in overlap and statistical power presented here after fCBA are in fact an underestimation of the potential of this method. This is caused by the small amount of data used to label the chosen regions (as few as 50 volumes for the hMT+ localiser after splitting), resulting likely in some inter-subject variability not normally observed when using full data sets.

A major source of loss of statistical power in group studies is due to the inherent differences in anatomy of individual subjects (Thirion et al., 2007). Advanced alignment schemes, either in volume space (Ashburner, 2007) or on the surface (Fischl et al., 1999; Goebel et al., 2006; Van Essen, 2005) are 
able to reduce this variance in anatomy. Functional regions are bound to the anatomy of the brain, however, even after macro-anatomical differences have been minimised, not all functional areas are brought into alignment. Indeed it is unlikely that alignment based on curvature, manually labelled landmarks or intensity values of T1 weighted images will be able to improve correspondence of functional regions much further as their location seems to be largely determined by micro-anatomical structure which is unobservable in vivo, with current technology with the exception of early sensory and motor areas which can be localised due to their increased levels of myelination (Glasser and Van Essen, 2011). For this reason, including functional regions in a group alignment schemes is a practical and powerful tool that can improve spatial correspondence of functional regions. Using ROI-based fCBA one is able to increase the power of group statistics, especially in functionally relevant regions, while allowing for whole brain analysis. 


\section{References}

Amunts, K., Zilles, K. (2001). Advances in cytoarchitectonic mapping of the human cerebral cortex. Neuroimaging Clin N Am 11, 151-169, vii.

Ashburner, J. (2007). A fast diffeomorphic image registration algorithm. Neuroimage 38, 95-113.

Duncan, K.J., Pattamadilok, C., Knierim, I., Devlin, J.T. (2009). Consistency and variability in functional localisers. Neuroimage 46, 1018-1026.

Evans, A.C., Marrett, S., Neelin, P., Collins, L., Worsley, K., Dai, W., Milot, S., Meyer, E., Bub, D. (1992). Anatomical mapping of functional activation in stereotactic coordinate space. Neuroimage 1, 43-53.

Fischl, B., Sereno, M.I., Tootell, R.B., Dale, A.M. (1999). High-resolution intersubject averaging and a coordinate system for the cortical surface. Hum Brain Mapp 8, 272-284.

Friston, K.J., Henson, R.N. (2006). Commentary on: Divide and conquer; a defence of functional localisers. Neuroimage 30, 1097-1099.

Friston, K.J., Rotshtein, P., Geng, J.J., Sterzer, P., Henson, R.N. (2006). A critique of functional localisers. Neuroimage 30, 1077-1087.

Frost, M.A., Goebel, R. (2012). Measuring structural-functional correspondence: Spatial variability of specialised brain regions after macro-anatomical alignment. Neuroimage 59, 1369-1381.

Glasser, M.F., Van Essen, D.C. (2011). Mapping human cortical areas in vivo based on myelin content as revealed by T1- and T2-weighted MRI. $J$ Neurosci 31, 11597-11616.

Goebel, R., Esposito, F., Formisano, E. (2006). Analysis of functional image analysis contest (FIAC) data with brainvoyager QX: From single-subject to 
cortically aligned group general linear model analysis and self-organizing group independent component analysis. Hum Brain Mapp 27, 392-401.

Goebel, R., Khorram-Sefat, D., Muckli, L., Hacker, H., Singer, W. (1998). The constructive nature of vision: direct evidence from functional magnetic resonance imaging studies of apparent motion and motion imagery. Eur $J$ Neurosci 10, 1563-1573.

Klein, A., Andersson, J., Ardekani, B.A., Ashburner, J., Avants, B., Chiang, M.C., Christensen, G.E., Collins, D.L., Gee, J., Hellier, P., Song, J.H., Jenkinson, M., Lepage, C., Rueckert, D., Thompson, P., Vercauteren, T., Woods, R.P., Mann, J.J., Parsey, R.V. (2009). Evaluation of 14 nonlinear deformation algorithms applied to human brain MRI registration. Neuroimage 46, 786-802.

Kriegeskorte, N., Goebel, R. (2001). An efficient algorithm for topologically correct segmentation of the cortical sheet in anatomical mr volumes. Neuroimage 14, 329-346.

Pantazis, D., Joshi, A., Jiang, J., Shattuck, D.W., Bernstein, L.E., Damasio, H., Leahy, R.M. (2010). Comparison of landmark-based and automatic methods for cortical surface registration. Neuroimage 49, 2479-2493.

Paus, T. (1996). Location and function of the human frontal eye-field: a selective review. Neuropsychologia 34, 475-483.

Peelen, M.V., Downing, P.E. (2005). Within-subject reproducibility of category-specific visual activation with functional MRI. Hum Brain Mapp 25, 402-408.

Sabuncu, M.R., Singer, B.D., Conroy, B., Bryan, R.E., Ramadge, P.J., Haxby, J.V. (2010). Function-based intersubject alignment of human cortical anatomy. Cereb Cortex 20, 130-140.

Saxe, R., Brett, M., Kanwisher, N. (2006). Divide and conquer: a defense of functional localizers. Neuroimage 30, 1088-1096; discussion 1097-1089. 
Sereno, M.I., Dale, A.M., Reppas, J.B., Kwong, K.K., Belliveau, J.W., Brady, T.J., Rosen, B.R., Tootell, R.B. (1995). Borders of multiple visual areas in humans revealed by functional magnetic resonance imaging. Science 268, 889-893.

Talairach, J., Tournoux, P. (1980). Co-planar sterotaxic atlas of the human brain. Thieme, Stuttgart.

Thirion, B., Pinel, P., Meriaux, S., Roche, A., Dehaene, S., Poline, J.B. (2007). Analysis of a large fMRI cohort: Statistical and methodological issues for group analyses. Neuroimage 35, 105-120.

Van Essen, D.C. (2005). A Population-Average, Landmark- and Surfacebased (PALS) atlas of human cerebral cortex. Neuroimage 28, 635-662.

Van Essen, D.C., Drury, H.A. (1997). Structural and functional analyses of human cerebral cortex using a surface-based atlas. J Neurosci 17, 70797102.

Weiner, K.S., Grill-Spector, K. (2010). Sparsely-distributed organization of face and limb activations in human ventral temporal cortex. Neuroimage 52, $1559-1573$ 


\section{AN INVESTIGATION INTO THE STRUCTURAL AND FUNCTIONAL DEVELOPMENT OF HUMAN SUPERIOR TEMPORAL CORTEX}

Corresponding publication:

Bonte, M., Frost, M.A., Rutten, S., Ley, A., Formisano, E. \& Goebel, R. (2012). An Investigation into the Structural and Functional Development of Human Superior Temporal Cortex. In preparation. 


\begin{abstract}
Here we present an investigation of the structural and functional development of auditory regions using many of the analysis approached developed for the previous chapters. To this end we analysed superior temporal anatomical landmarks and measured fMRI responses while children (8-9 years), adolescents (14-15 years) and adults passively listened to voices, object sounds and amplitude modulated tones. Anatomical landmarks show a developmental increase in inter-individual variability in right hemisphere superior temporal areas (STS, FTS, planum temporale), and stable interindividual variability in the left hemisphere. The development of functional regions resembles that of anatomical regions with an increase in inter-subject variability observed in the right hemisphere, but not the left, of some functional regions involved in auditory processing. It is likely that this development continues as a result of the exposure to sounds we experience in our everyday environment.
\end{abstract}




\section{Introduction}

The human superior temporal cortex is characterized by high interhemispheric and inter-individual variability in terms of anatomy and function and shows a long-lasting developmental time course until early adulthood. It includes one of the most known anatomical asymmetries of the brain, a left larger than right planum temporale (PT), which is (Brodmann, 1909) typically assumed to relate to a famous functional asymmetry, the left hemispheric specialization for language (Geschwind and Levitsky, 1968). A leftward asymmetry is also consistently reported for earlier auditory areas, especially in terms of larger white matter volume below the left Heschl's gyrus (HG) (Penhune et al., 1996). Rightward superior temporal asymmetries are less well-known, but recent studies have suggested a deeper right than left superior temporal sulcus (STS), especially in its posterior section prior to splitting in the ascending and descending branches (Barrick et al., 2005 Ochiai et al., 2004; van Essen et al., 2005; Watkins et al., 2001).

In addition to this inter-hemispheric variability, superior temporal cortex also shows large inter-individual variability, including variability in the number of HGs, ranging between one to three per individual hemisphere (Abdul-Kareem and Sluming, 2008; Campain and Minckler, 1976), size and form of the planum temporale (Westbury et al., 1999) and folding pattern of the STS (Im et al., 2010; Ochiai et al., 2004). An important and largely unanswered question is how this anatomical variability relates to individual differences in brain function.

A left > right asymmetry of the posterior Sylvian fissure and/or PT as well as a deeper right STS have been found to be already present in newborns and young infants (Dubois et al., 2010; Glasel et al., 2011; Hill et al., 2010), suggesting a strong genetic component underlying these anatomical asymmetries. On top of these innate predispositions, superior temporal regions show continued developmental changes during childhood and adolescence. Throughout this age period, grey matter volume of the STS and posterior superior temporal gyrus (STG) continues to increase (Sowel et al., 2003) as well as the left > right asymmetry of the Sylvian fissure (Sowel et al., 2002).

Most of the upper surface of the superior temporal cortex and adjacent regions of the STS strongly respond to sound and are involved in basic and 
higher order auditory perception. Over the last decade, a key issue in neuroimaging research has been the investigation of specialized superior temporal areas/pathways for the processing of different auditory categories. This research suggests a functional segregation of areas specialized for the processing of (1) speech sounds in bilateral mid-posterior (Binder et al., 2000, Hickok and Poeppel, 2007) and/or left anterior (Rauschecker and Scott, 2009) superior temporal cortex, and of (2) human voice sounds along the STG/STS, with strong voice selectivity especially in the right anterior STS (Belin et al., 2000). Like anatomical asymmetries, these functional asymmetries seem also to be present in infants. Three month old infants show bilateral, but stronger left temporal activity to speech and speech-like sounds (Dehaene-Lambertz et al., 2002). Seven but not 4 month old infants show stronger right superior temporal activity to voices as compared to non-vocal sounds (Grossmann et al., 2010). Electrophysiological (EEG; Sharma et al., 1997; Bonte and Blomert 2004) evidence indicates that the neural processing of speech further develops and refines during childhood. Although 3-4 year olds show early voice selective EEG responses (Rogier et al., 2010), little is known about its further development.

How normal inter-hemispheric and inter-individual variation relates to brain function remains largely elusive, especially for areas beyond early auditory cortex. In adults, volume of the left HG correlates with functional activation to temporally modulated noise and volume of the right $\mathrm{HG}$ with functional activation to spectrally modulated noise (Warrier et al., 2009). Furthermore, larger grey matter volume of the left HG relates to the ability to learn linguistic pitch contours (Wong et al., 2008), and larger white-matter density of the left $\mathrm{HG}$ relates to individual differences in the learning of foreign speech sounds (Golestani et al., 2006). Finally, rightward asymmetry of HG grey matter volume and functional activation to harmonic complex sounds were found to relate to a preference for spectral as compared to as compared to fundamental (F0) pitch perception (Schneider et al., 2005). In contrast, left asymmetry of the planum temporale was found not to predict language lateralisation as measured with the intracarotid Sodium Amytal procedure (Dorsaint-Pierre et al., 2006) or fMRI activity during listening to words (Binder et al., 1996). Recent evidence further suggests that the strength of structurefunction relations, in this case between FM detection threshold and left HG gray matter density, may also change during development (Sutherland et al., 2012). 
Here we investigate the functional and structural development of superior temporal cortex in children, adolescents and adults by analyzing interhemispheric and inter-individual variability in tone and voice selective fMRI responses and anatomical landmarks. Analysis of structural and functional variability was performed on the cortical surface (Frost \& Goebel, 2012). Structural variability was quantified by manually labelling sulcal and gyral landmarks on each individual's surface before combining all from each age group on a group surface and computing inter-subject distances. In order to avoid the confounding factor of macro-anatomical variability when assessing functional variability, measures of functional variability were quantified on fMRI activity after cortex-based group alignment.

\section{Methods}

\subsection{Participants}

(f)MRI measurements were performed in thirty-eight healthy Dutchspeaking participants ( 13 children, 14 adolescents, 10 adults). Data of 3 children and 4 adolescents were discarded: 2 children did not complete the measurement, 1 child moved too much, 1 adolescent was discarded due to technical problems during the measurement, 3 additional adolescents showed no significant activation to tones and/or voices. Data of 30 subjects were included in the analysis: 10 children (8f, mean age $9.0 \pm 0.66$ years), 10 adolescents (8f, mean age $14.1 \pm 0.59$ ) and 10 adults (6f, mean age $24.0 \pm 2.39$ years). Adults and adolescents received a monetary reward for participation, children were given a present. Informed consent was obtained from all adult and adolescent participants and from parents of adolescents and children, according to the approval by the Ethical Committee of the Faculty of Psychology and Neuroscience at the University of Maastricht.

All participants were Dutch speakers with normal hearing as assessed with a pure tone audiogram (detection thresholds of frequencies from $250-8000 \mathrm{~Hz}$ at 0-20 decibels). Handedness was assessed with Annett's Handedness Questionnaire (Annett, 1979). Except for one child and one adult, all participants were right handed. Psychometric tests included a word reading task, a phoneme deletion task and memory span task (Vaessen \& Blomert, 2010), as well as the block design, similarities and digit span sub-tests of the WISC (Van der Steene, Vertommen, and Bleichrodt, 2000) or WAIS (Kort, et al., 2005). With the exception of two children and one adolescent who scored 
around 1.5 SD below the standardized mean on the memory span task, all children, adolescents and adults scored within normal limits on each of these psychometric tests.

\subsection{Anatomical Landmarks}

\subsubsection{Anatomical measurement}

Brain Imaging was performed with a 3 Tesla scanner (head setup) at the Maastricht Brain Imaging Center. All children and adolescents were acquainted to the scanning environment and trained to minimize head movement using a simulation scanner. A high-resolution structural scan (voxel size, $1 \times 1 \times 1 \mathrm{~mm}^{3}$ ) was also collected for each subject using a T1-weighted three-dimensional ADNI sequence [TR, $2050 \mathrm{~ms}$; echo time (TE), $2.6 \mathrm{~ms}$; 192 sagittal slices].

\subsubsection{Cortex Based Alignment}

Anatomical and fMRI (see below) data were analysed using BrainVoyager QX 2.4 (Brain Innovation). The anatomical images of each participant were normalized into Talairach space. This operation included the definition of the landmarks AC (anterior commissure), PC (posterior commissure) as well as a demarcation of the extremities of the cortex (inferior, superior left and right most point); the defined subject-specific landmarks were then used to rotate each brain in the AC-PC plane followed by piecewise, linear transformations to fit each brain in the common Talairach "proportional grid" system (Talairach and Tournoux, 1980). Mesh representations of the cortical surface were created using an automatic segmentation tool (Kriegeskorte and Goebel, 2001). Curvature maps were then derived from the extracted cortical meshes for both hemispheres of each individual. Cortex based alignment (CBA) was then performed by first carrying out a rigid alignment to a randomly chosen target subject for each group to minimize differences in rotation and orientation of the spherical surface reconstructions. This was followed by a non-linear morphing alignment to a dynamic group average curvature map. This was done in a coarse to fine fashion where during the early phase, smoothed curvature maps are used to align the gross anatomical features (central sulcus, Sylvian fissure, superior temporal sulcus etc). As the alignment algorithm progresses, more detailed curvature information is 
added to maximize macro-anatomical correspondence across the whole cortex (Frost and Goebel, 2012; Goebel et al., 2006). CBA results in the creation of values describing warping of each vertex of each hemisphere from its individual (Talairach) subject space, to the group aligned space. These values were used to co-register the functional data and anatomical path definitions to the group aligned space.

\subsubsection{Landmark definition}

Five anatomical landmarks in regions associated with auditory processing including areas involved in voice processing, were manually labelled and their spatial variability was measured both before and after macroanatomical alignment. The five landmarks are illustrated in figure 1 and consist of the superior temporal sulcus (STS), the superior temporal gyrus (STG), the first transverse sulcus (FTS), Heschl's sulcus (HS) and planum temporale (PT). The landmarks were identified and labelled according to the following criteria (Frost and Goebel, 2012; Kim et al., 2001; Pantazis et al., 2010). (1) The superior temporal sulcus separates the superior from the middle temporal gyrus and was traced from its anterior border at or near the temporal pole until its posterior split into an ascending and descending (or horizontal) branch. (2) The superior temporal gyrus runs parallel to the lateral sulcus (Sylvian fissure) and the STS, and was traced from its anterior border at the temporal pole to its posterior border at the temporal parietal junction. (3) The first transverse sulcus runs obliquely across the upper surface of the STG, and forms the anterior medial border of Heschl's gyrus (HG). FTS was traced from its origin near the retroinsular region, near the medial border of HG until its endpoint on the planum polare. (4) Heschl's sulcus forms the posterior limit of HG and was traced from the medial border of HG until its antero-lateral border on the superior temporal surface or at the lateral rim of the STG. (5) The planum temporale (PT) is a triangular region on the superior temporal surface posterior to HG. Following Kim et al., (2001) its anterior-medial border was defined by the HS, or alternatively by the sulcus intermedius (SI) in case it was present (See Fig. 1). The SI is defined as a sulcus not originating from the retroinsular region that incompletely divides HG. Laterally the PT was confined by the lateral rim of the STG. The posterior border was traced from the medial origin of HS through the deepest point of the Sylvian fissure until the most posterior point of the STG (at the temporal parietal junction). 


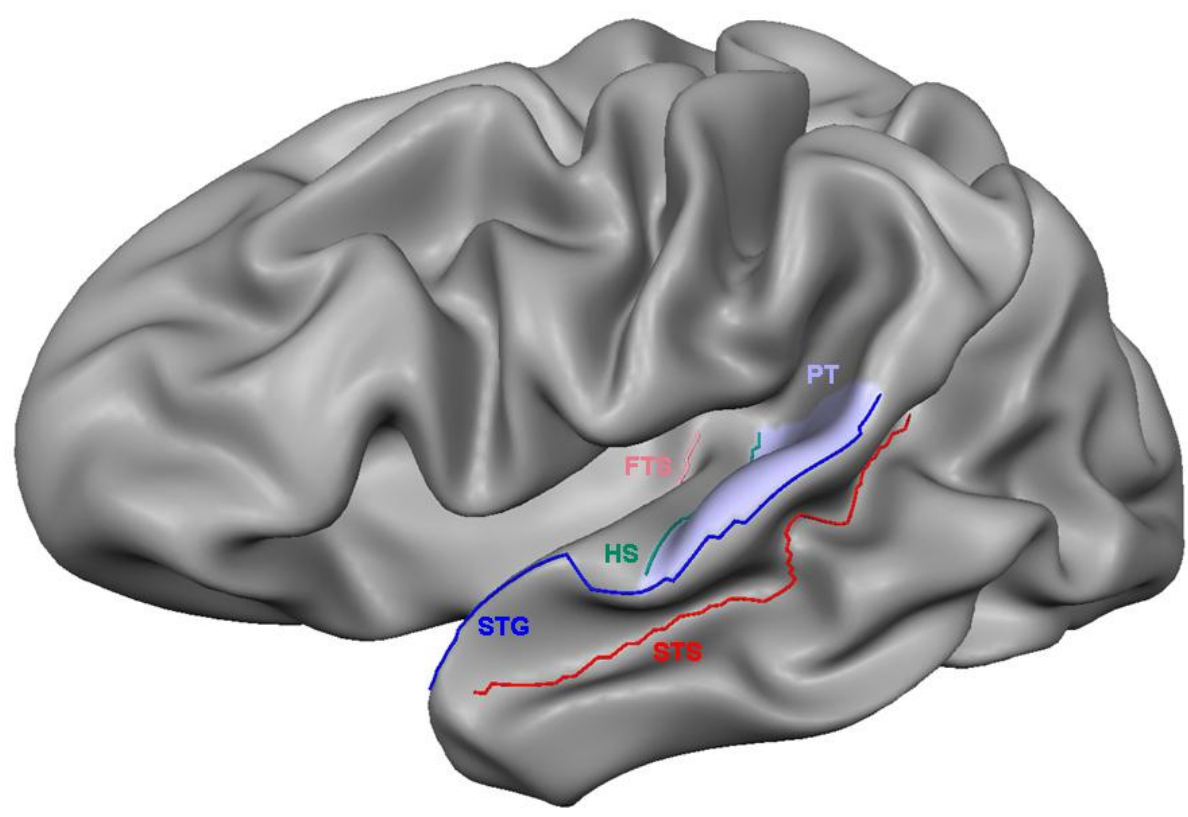

Figure 1. The defined anatomical landmarks. Pink $=$ FTS, Green $=$ HS, Blue $=$ STG, Red $=$ STS. The light blue patch of cortex is the PT.

\subsubsection{Quantification / statistical analysis}

To quantify anatomical variability of sulcal and gyral paths across subjects, a modified Hausdorff distance measure (Pantazis et al., 2010) was used to calculate pair wise distances between each subject, within each age group. Due to the fact that the planum temporale is an area of cortical surface and cannot be described as a path (gyrus or sulcus), inter-subject variability was quantified using the following measure of overlap:

$$
\mathrm{o}_{\mathrm{v}}=N_{\mathrm{v}} / N
$$

where $\mathrm{N}_{\mathrm{v}}$ is the number of subjects whose planum temporale occupies a certain vertex and $\mathrm{N}$ is the number of subjects 
Sulcal depth values were sampled for each vertex of a subject's folded mesh representation from a sulcal depth volume map measuring the shortest distance of each voxel of a white/grey matter segmentation to the brain surface. The shortest distance was calculated using a distance transform operation starting at the border of a (morphologically) closed brain segmentation.

\subsection{Functional localisers}

\subsubsection{Functional MRI measurements}

For each subject, an 8 minute functional run $(3 \mathrm{~mm} \times 3 \mathrm{~mm} \times 3 \mathrm{~mm})$ was collected using a standard echoplanar- imaging (EPI) sequence (repetition time $[\mathrm{TR}]=3.0 \mathrm{~s}$; acquisition time $[\mathrm{TA}]=2.0 \mathrm{~s}$, field of view $[\mathrm{FOV}]=192$ $\mathrm{mm} \times 192 \mathrm{~mm}$, matrix size = 64 x 64, echo time [TE] = $32 \mathrm{~ms}$ ). Each volume consisted of 33 slices (distance factor 10\%), including the temporal cortex. During the measurements, subjects listened to auditory stimuli that were presented binaurally and at a comfortable listening level via MR compatible headphones in the 1000-ms silent gap between two volume acquisitions. The functional run consisted of 12 stimulation blocks (18 s / 6 volumes) alternated with $12 \mathrm{~s}$ (4 volumes) rest. During the stimulation blocks, participants listened to either (1) vocal sounds (speech and non-speech), (2) object sounds (musical instruments, environmental and animal sounds), both adapted from Belin et al., (2000), or (3) tones (amplitude modulated (8 Hz) tones ranging from $0.3-3 \mathrm{kHz}$ ).

\subsubsection{Voice selective responses and responses to tones}

fMRI data were subjected to conventional pre-processing in BrainVoyager QX 2.4, including high-pass temporal filtering and motion correction (see Goebel et al., 2006). To identify individual regions of interest, functional data were spatially smoothed to $4 \mathrm{~mm} \times 4 \mathrm{~mm} \times 4 \mathrm{~mm}$ and a general linear model (GLM) analysis was performed on time course data sampled on individual cortex meshes. In order to map fMRI signal time courses from volume space to surface space, values located between the grey/white matter boundary and up to $4 \mathrm{~mm}$ into grey matter towards the pial surface were sampled with trilinear interpolation and averaged resulting in a single value for each vertex of a cortex mesh. A resulting region of interest is therefore called a patch of interest (POI). Voice and Tone POIs were selected by label- 
ling significantly active surface vertices within the superior temporal cortex region. Voice POIs were labelled using the contrast voices $>$ (objects + tones) / 2. Tone POIs were labelled using the contrast tones > baseline.

\subsubsection{Quantification / statistical analysis}

In order to observe structural-functional relationships in auditory areas we measured inter-subject spatial variability of functional regions after macroanatomical variability had been minimized using CBA. The rationale being that, without accounting for anatomical differences, variability in location of functional regions would be confounded by the variability in the underlying anatomy. Having minimised macro-anatomical variability one can measure the true, structural functional correspondence. For this reason before functional variability was measured, individual maps were brought into their corresponding group aligned space, which was achieved through CBA. To quantify differences in location of a range of auditory regions, the geometric centre of each POI was calculated and inter-subject distances were measured. A POI is a surface patch consisting of a collection of $n$ edge-connected triangles. To calculate the centroid, $C$ of a POI, the center coordinates of each triangle $c_{i}$ (mean of three triangle vertices) was averaged weighted by the area $A_{i}$ of the respective triangle:

$$
C=\sum_{n} A_{i} C_{i} / \sum_{n} A_{i}
$$

Since a POI is not necessarily a plane, the centroid might fall above or below the surface patch. To identify the centroid vertex $C_{v}$ of the POI the centroid is finally projected onto the surface patch using the normal $N$ that is obtained by averaging the normals $N_{i}$ of the constituting triangles weighted by the respective area:

$$
N=\sum_{n} A_{i} N_{i} / \sum_{N} A_{i}
$$

The centroid vertex $C_{v}$ is finally specified as the vertex of the POI with the minimal distance to the normal line.

After calculating the centroid vertex of the POI of a homologue functional area for each subject, the group POI center was calculated by choosing the group vertex with the shortest mean distance to the subject's centroid verti- 
ces. The shortest distance (geodesic) between two vertices was calculated on the folded group mesh using the Dijkstra algorithm (e.g. (Cormen et al., 2001) Section 24.3: Dijkstra's algorithm. pp. 595-601.). The smaller this distance, the closer in space the functional regions are (after accounting for anatomical differences), and the higher the distance value the greater variability in location of this functional region.

\section{Results and Discussion}

We investigated structural and functional development of superior temporal cortex from childhood to adulthood by analysing inter-hemispheric and inter-individual variability in anatomical landmarks and fMRI responses to tones and voices. By applying advanced analysis tools to individually determined markers we zoomed-in into these landmarks and activation patterns and show structural and functional indices of continued development of superior temporal cortex from childhood to adulthood.

\subsection{Inter-individual variability - anatomical landmarks Inter- individual variability - anatomical landmarks}

Structural development was investigated by tracing the STS, STG, FTS and HS and by delineating the PT, all areas involved in auditory processing and including voice selective areas (Belin et al., 2000). Inter-hemispheric and inter-individual variability as assed by the Hausdorff distance measure indicates that there is a larger variance in the right hemisphere of adults than in the left hemisphere. This overall left-right asymmetry is not as pronounced in lower age groups (See figure 2) and is therefore likely to be a feature which develops over time. 
$\mathrm{LH}$

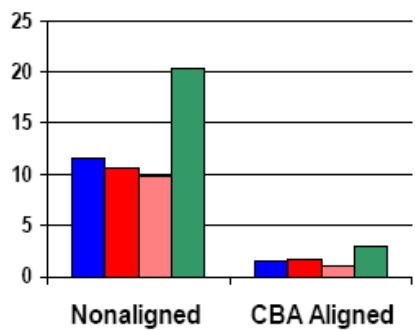

Adults

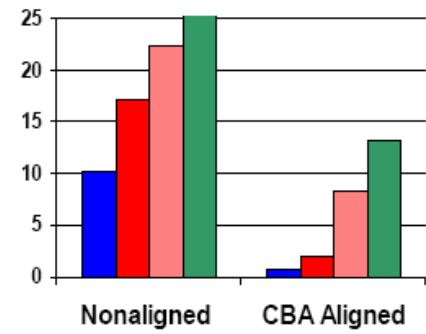

Adolescents

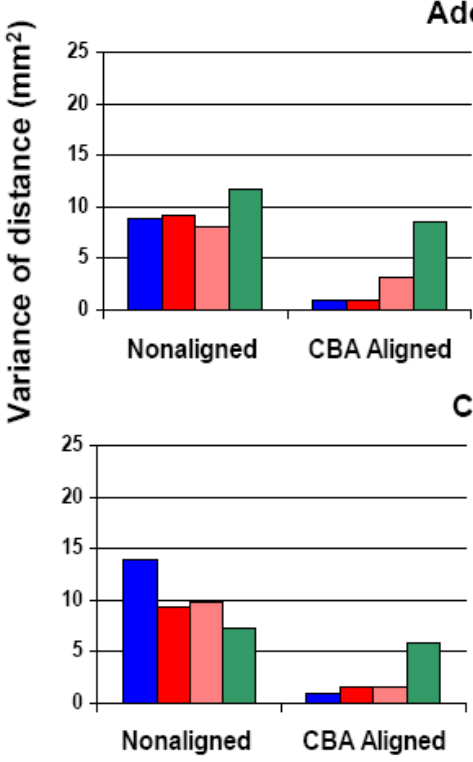

$\mathrm{RH}$
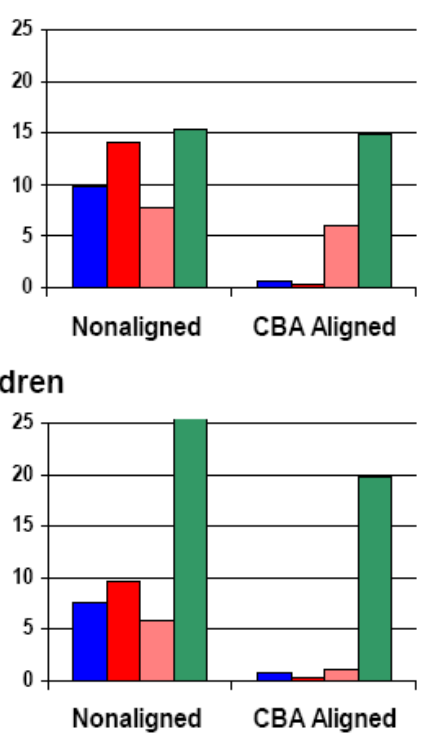

Figure 2. Bar graph depicting the inter-subject distance between the four manually defined anatomical sulcal and gyral landmarks. Pink $=$ FTS, Green $=$ HS, Blue $=$ STG, Red $=$ STS .

CBA is able to greatly reduce anatomical variance as seen in the right side of each graph of figure 2, however some variance remains, especially in the right hemisphere. Due to the presence of a SI in some subjects, distance measures of HS are not trustworthy as in some cases distances are between the HS of one subject and the SI of another. For this reason further detailed analysis of HS was not pursued. 


\section{Mean inter-subject path distance}

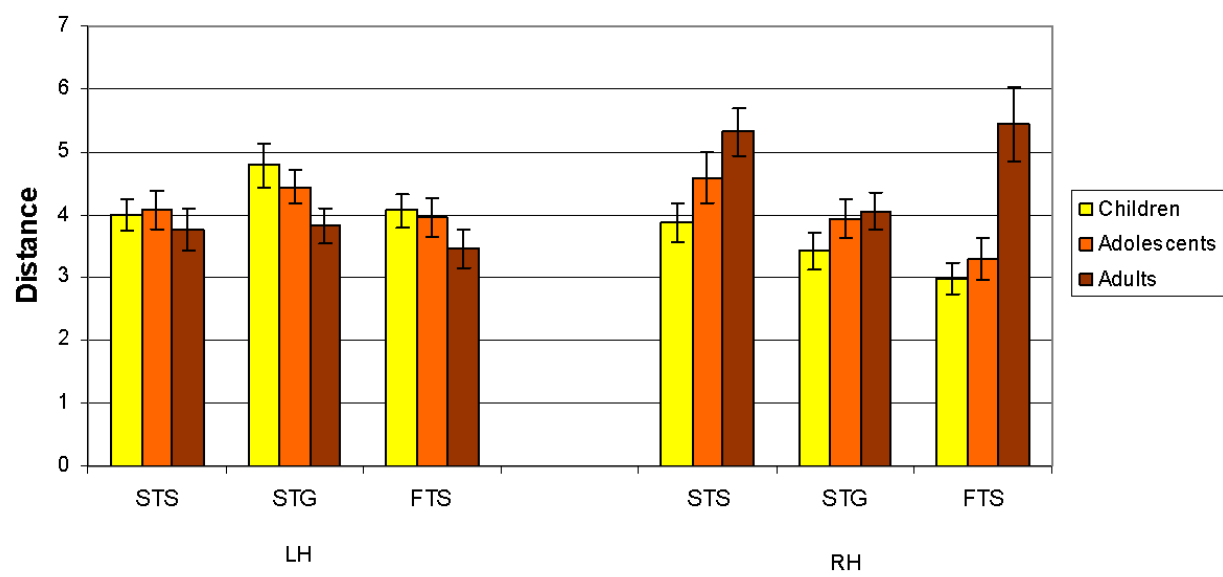

Figure 3. Mean distances between 3 anatomical landmarks in both hemispheres, across age groups. Inter-subject variability increased with age in the right hemisphere (right graph) but not in the left hemisphere (left graph).

Owing to the fact that, when using a Hausdorff distance measure, variances are computed from 0 in absolute terms, in a pair wise fashion and not to a mean group landmark (this is done to avoid the situation where if all subjects have a large distance between each other, but this difference is equal between each subject, then this would result in a variance of 0 despite the large inter-subject variability in location of the landmark). As a consequence however, statistical tests are difficult as there is no mean and variance of the measurement. To overcome this problem the following analysis was based on the 45 pair wise distances between 10 participants per region per age group from. As these measurements are compared across age groups, not between hemispheres, and are independent from each other, so mean distances between subjects can be derived as well as the variance about that mean. Statistical analysis of these distance measures showed that there was a significant effect of age in the right hemisphere, but not in the left hemisphere for the STS $(\mathrm{F}(2,132)=3.857, \mathrm{p}<0.05)$ and FTS $(\mathrm{F}(2,132)=10.516$, $\mathrm{p}<0.05$ ) however this was not significant for the STG (See figure 3). This is further evidence that the increased variability of anatomy in the right superior temporal cortex (STS and FTS), is a feature of development and suggests relatively strong, experience-dependent changes in the right as compared to the left hemisphere (Yoon et al., 2010). 


\subsection{Size of PT}

Planum temporale size was measured on each subject's cortical mesh representation. Because all brains were normalised to the Talairach template, differences in size of brains between age groups are removed allowing comparison of the degree of lateralisation to be made. As can be seen in figure 4, all age groups showed the expected left larger than right PT. A repeated measures ANOVA lead to a main effect of hemisphere $(\mathrm{F}(1,27)=58.2$, $\mathrm{p}<0.001$ ) confirming this left>right asymmetry, and no significant hemisphere-by-group interaction $(F(2,27)=2.1$, n.s. $)$ indicating a stable asymmetry across age groups.

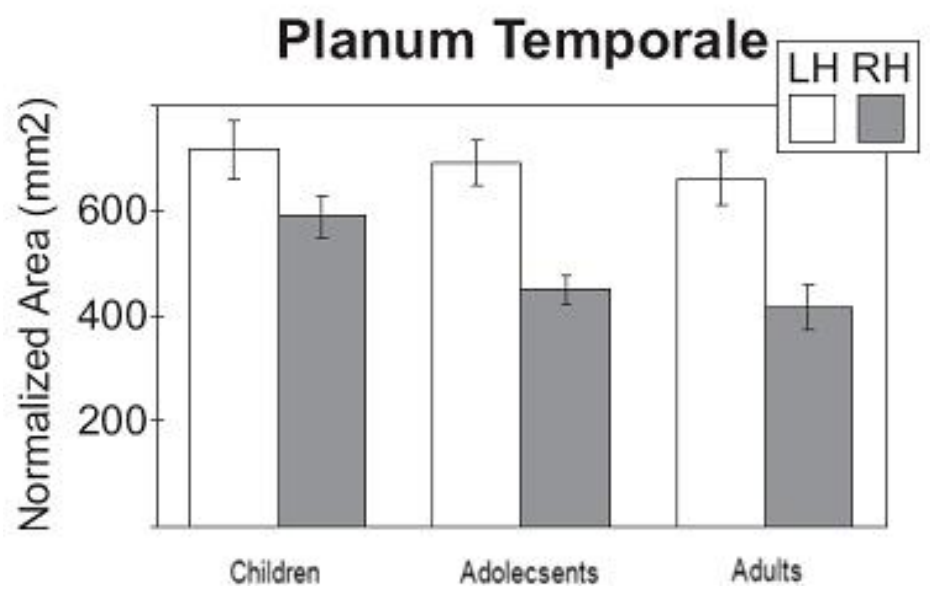

Figure 4. Bar graph depicting size of PT in left and right hemispheres across all three age groups. In both adolescents and adults the PT in the right hemisphere is significantly smaller than the PT in the left hemisphere.

\subsection{Sulcal Depth}

Besides the relatively well known left larger than right PT asymmetry, more recent studies also indicated an asymmetry of the STS, and specifically a larger sulcal depth of the posterior portion of the STS prior splitting in its ascending and descending branches (Barrick et al., 2005; Dubois et al., 2010; Glasel et al., 2011; Hill et al., 2010; Ochiai et al., 2004; van Essen et al., 2005; Watkins et al., 2001). Sulcal depth along STS is illustrated in figure 5 for children, adolescents and adults. Each of the three age groups showed the expected rightward asymmetry with a deeper right STS in the two segments 
(mid and posterior) posterior to Heschl's gyrus / Heschl's sulcus. In agreement, our statistical analysis showed significant main effects of hemisphere $(F(1,27)=12.0, p<0.005)$ and segment $(F(1,54)=163.4, p<0.001)$ and a significant hemisphere-by-segment interaction $(F(2,54)=17.2, p<0.001)$, followed by significant main effects of hemisphere in the mid $(F(1,27)=8.1$, $\mathrm{p}<0.01)$ and posterior $(\mathrm{F}(1,27)=22.2, \mathrm{p}<0.001)$, but not in the anterior segment $(\mathrm{F}(1,27)=3.2$, n.s. $)$, and no hemisphere-by-group interactions within the separate segments. 

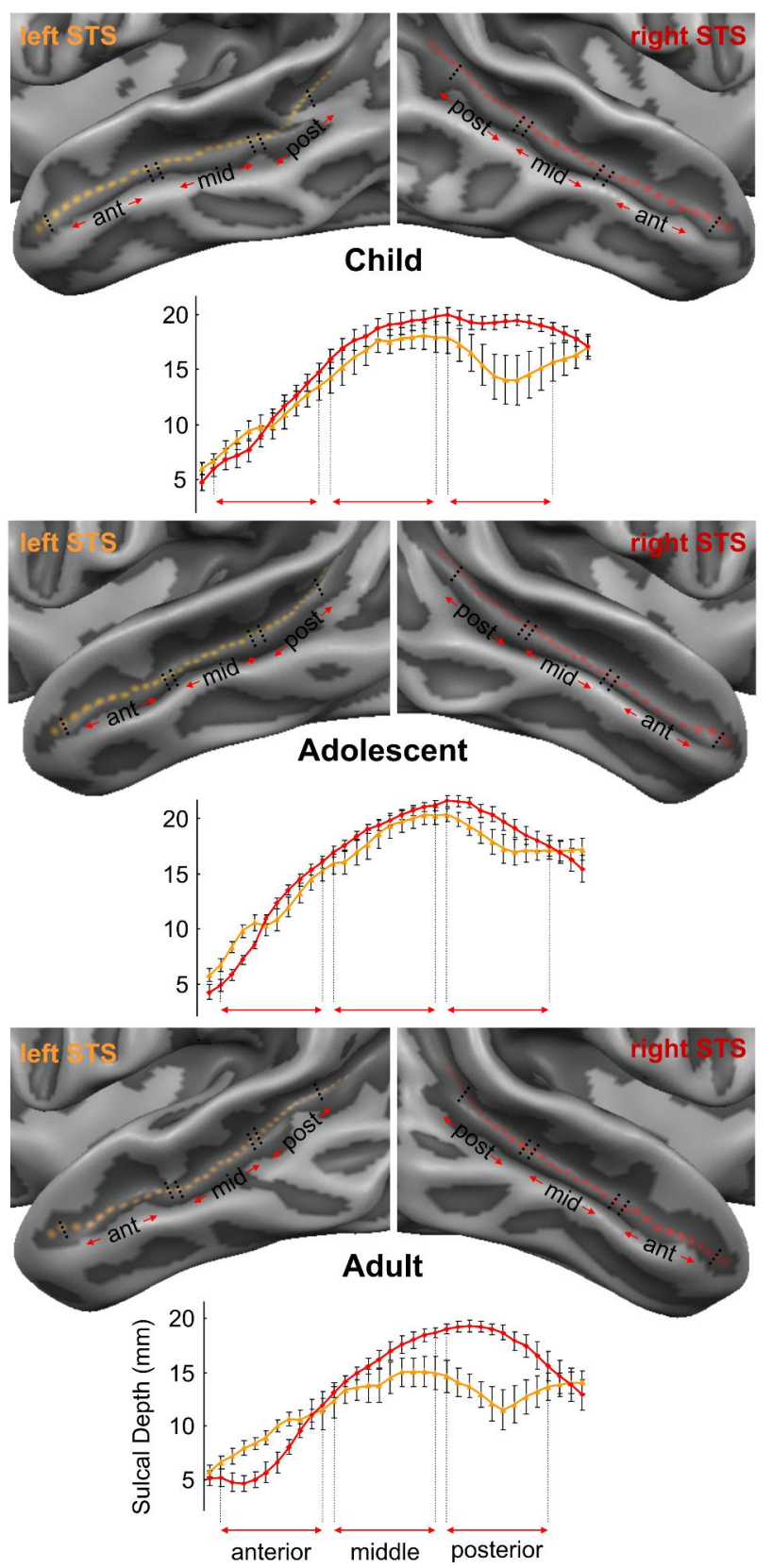

Figure 5. Sulcal depth (mm) along the left and right STS across all three age groups. The length of the STS has been divided into three sections; anterior, mid, and posterior segment. 


\subsection{Inter-individual variability - Tone and Voice selective regions}

Having observed increasing evidence of a development of lateralised differences in the anatomy of auditory regions we now turn our attention to functional measures of audition and whether there are similar hemispheric differences across age groups. In order to obtain a measure of functional variability, free from the confound of anatomical variability, the following results were all achieved after bringing the functional data into the corresponding group aligned space using CBA.

\section{Children}
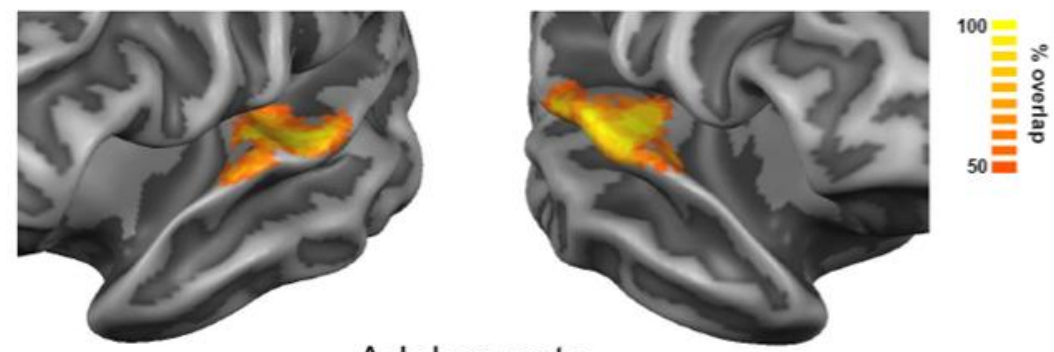

Adolescents

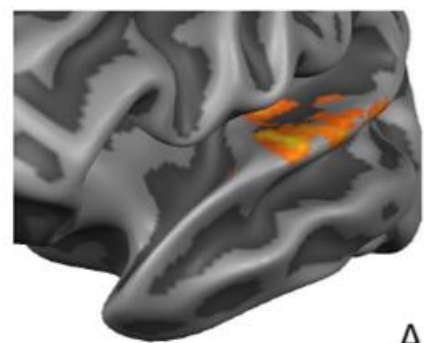

Adults
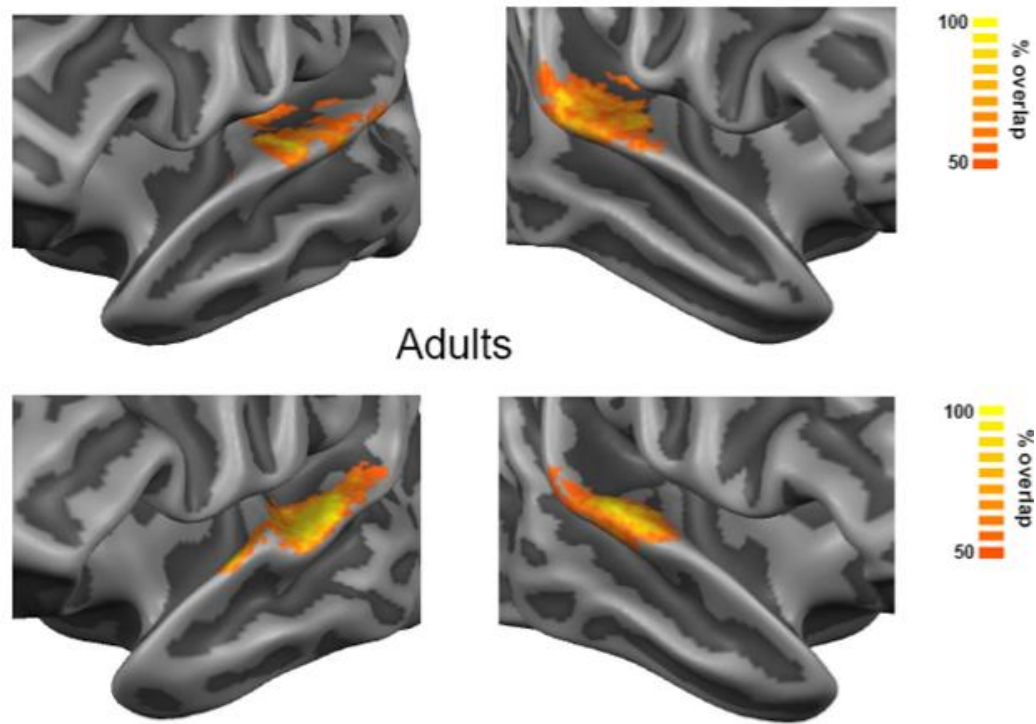

100

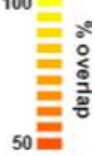

Figure 6. Probabilistic activation maps elicited by tones from children, adolescents in the left and right hemisphere. The brighter the yellow the greater inter-subject overlap of the functional region responding to tones. The functional maps were aligned using CBA to reflect variability in spatial location uncontaminated by underlying anatomical variability. 
Figure 6 illustrates auditory cortical activity elicited by tones in the different age groups. As can be seen by the bar graph of figure 7, this auditory cortical activity exhibited the same pattern across all three age groups namely, larger variability in the right hemisphere than the left hemisphere. While only the children group showed significant differences between left and right hemispheres $(\mathrm{p}=0.02)$, the other groups approached significance and the results indicate a similar trend in the data. This is somewhat of a surprise as this functional region is most associated with PT which showed a difference in lateralisation across age groups with children showing no hemispheric difference, yet older age groups showed larger variability in the right hemisphere than the left (see figure 4).

\section{Distance to Centriod Freqencey Areas}

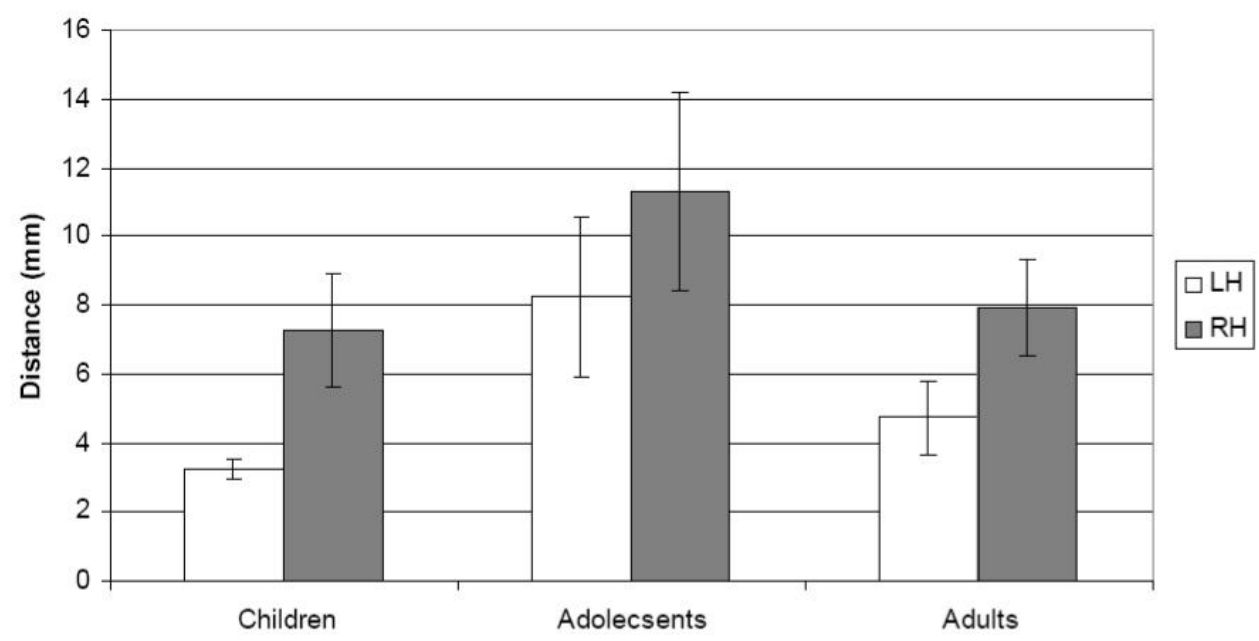

Figure 7. Bar graphs of the mean distance from group centre to individual centres of functional areas. Higher levels indicate greater spatial variability between subjects. 
Child
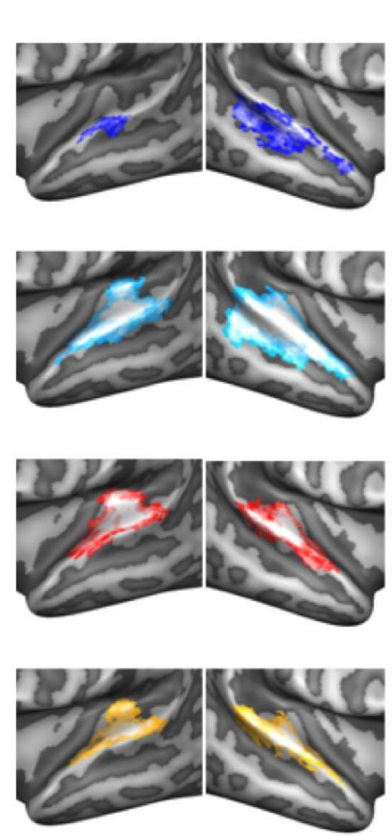

Adolescent

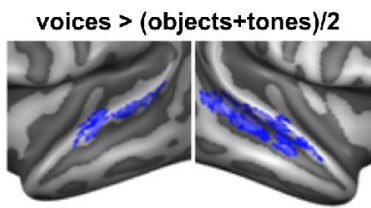

voices $>$ baseline

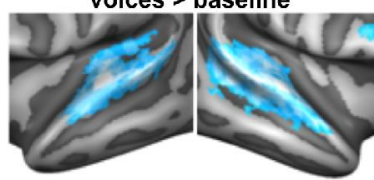

objects > baseline

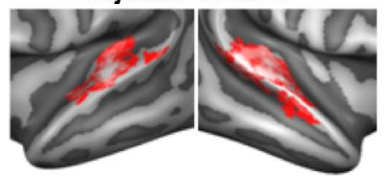

tones $>$ baseline

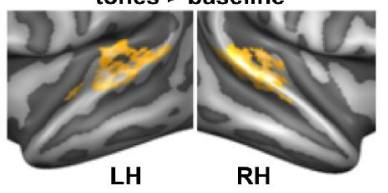

Adult
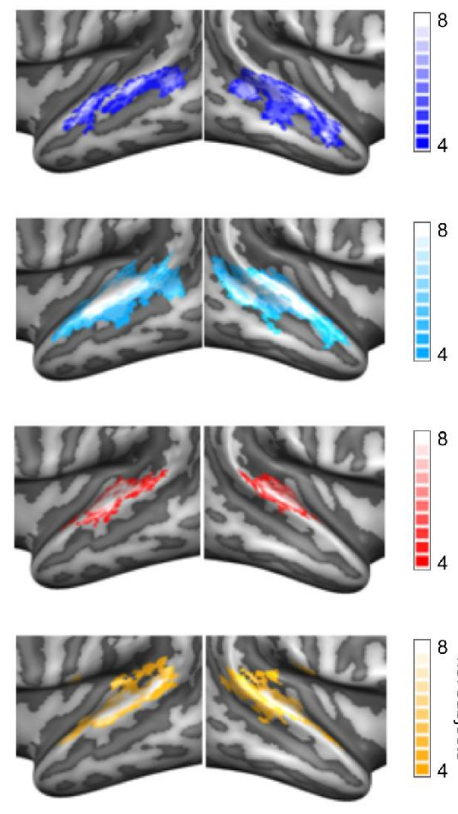

Figure 8. Probabilistic maps illustrating amount of subject overlap for left and right temporal activity to voices, objects and tones, as well as voice selective activity across the three age groups. Data of 8 children and a representative selection of 8 adolescents and 8 adults were included and the maps showing 50 to $100 \%$ subject overlap $(n=4$ to $n=8)$. Individual subject thresholds were set to $t=1.96(\mathrm{p}<0.05)$ for voice selective activity and $t=3.0(\mathrm{p}<0.003)$ for the three contrasts versus baseline. Individual cluster thresholds were set to $25 \mathrm{~mm} 2$, group cluster thresholds were set $\mathrm{t} 20 \mathrm{~mm}$.

We not only examined early auditory areas, but also voice selective areas to observe whether inter-subject variability in location of these areas changes between hemispheres and across development. As can be seen in figure 8, similar voice selectivity was found along the left and right STG/STS in children, adolescents and adults. Furthermore, this activity showed two consistent clusters in the left and right hemispheres: a posterior STS cluster poste- 
rior to HS and a mid STG/STS cluster at the level of $\mathrm{HG} \mathrm{HS}^{1}$. In the right hemisphere, most subjects also showed an additional STG/STS cluster anterior to HG. Preliminary analyses of inter-hemispheric and inter-individual variability in voice selectivity focused on the posterior and mid voice regions.

\footnotetext{
${ }^{1}$ The anterior cluster does not appear with the same level of reliability as the mid and posterior regions and therefore we focused on the mid and posterior clusters
} 
Distance to centriod posterior voice region

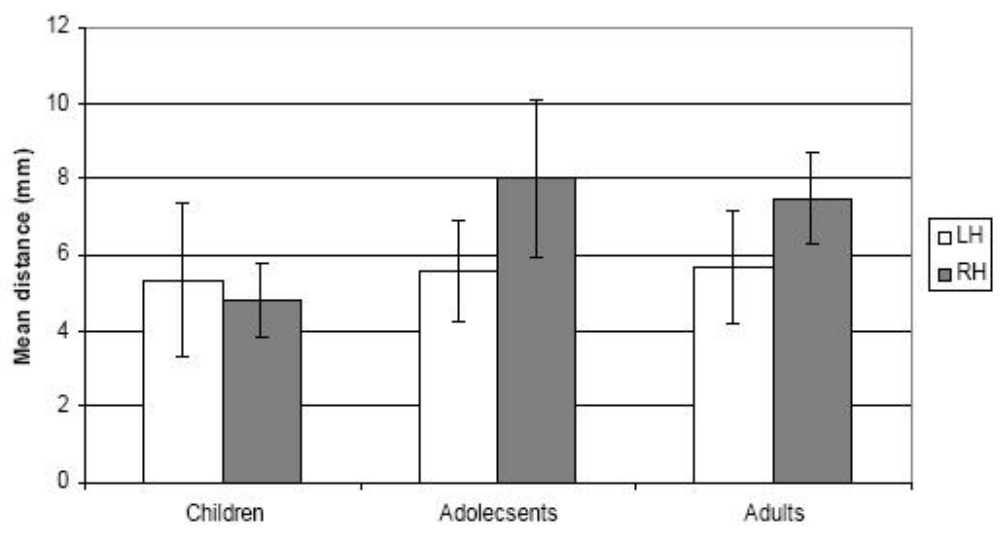

Distance to centiod mid voice region

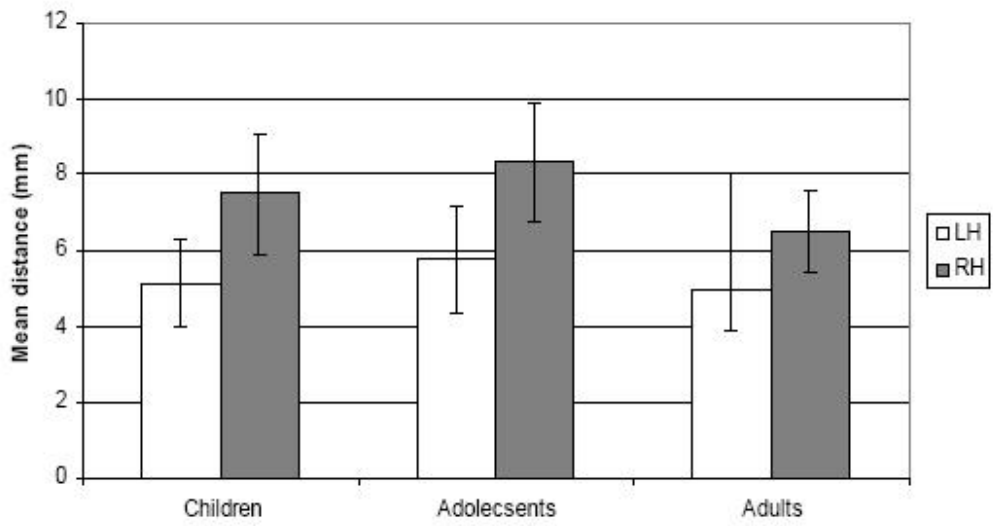

Figure 9. Bar graphs of the spatial variability of two voice selective functional regions.

While no significant results have been found, interesting trends in the functional data reflect some of the anatomical variability seen between hemispheres and across age groups (See figure 9). Similar to the activity elicited by tones, the mid voice regions show an increased variability in right when compared to left hemisphere across all age groups. In the posterior region however, the lateralised effect of increased right variability in the location of this function region is not as pronounced in the child group, yet is clearly present in the adolescent and adult group. This is in line with our anatomical findings as well as previously reported development of cortical anatomy 
where the posterior portion of the right STS is the site of the most significant change during development. Functionally, differences in exposure to more right-lateralised auditory categories such as music (Klein \& Zatorre, 2011) may contribute to this age-related increase in inter-subject variability. The absence of similar increases in homologue areas of the left hemisphere may be related to the lateralisation of language function to the left hemisphere, developing more consistently and strongly than for the right hemisphere.

\section{Conclusions}

Using advanced tools to label anatomical landmarks and quantify the intersubject variability of the spatial location of these landmarks we have observed that the increased variability of anatomical landmarks observed in adults is not present in children but develops over time. In order to carry out an investigation of variability of the spatial location of functional regions, we first minimised anatomical variability using CBA, an advanced, surface based alignment procedure. After successfully minimising macro-anatomical differences across subjects we have begun to see a picture emerging which indicates that the development of functional regions corresponds with the development of anatomical regions and increases in variability in organisation of auditory areas continues after childhood and through development through interactions with one's environment and the everyday expose to the vast auditory landscape around us. 


\section{References}

Abdul-Kareem, I. A., and Sluming, V. (2008) . "Heschl gyrus and its included primary auditory cortex: structural MRI studies in healthy and diseased subjects," J Magn Reson Imaging 28, 287-299.

Annett, J., Annett, M., Hudson, P.T., Turner, A. (1979) . The control of movement in the preferred and non-preferred hands. $Q J$ Exp Psychol 31, 641-652.

Barrick, T. R., Mackay, C. E., Prima, S., Maes, F., Vandermeulen, D., Crow, T. J., and Roberts, N. (2005) . "Automatic analysis of cerebral asymmetry: an exploratory study of the relationship between brain torque and planum temporale asymmetry," Neuroimage 24, 678-691.

Belin, P., Zatorre, R. J., Lafaille, P., Ahad, P., and Pike, B. (2000) . "Voiceselective areas in human auditory cortex," Nature 403, 309-312.

Binder, J. R., Frost, J. A., Hammeke, T. A., Bellgowan, P. S., Springer, J. A., Kaufman, J. N., and Possing, E. T. (2000) . "Human temporal lobe activation by speech and nonspeech sounds," Cereb Cortex 10, 512-528.

Binder, J. R., Frost, J. A., Hammeke, T. A., Rao, S. M., and Cox, R. W. (1996) . "Function of the left planum temporale in auditory and linguistic processing," Brain 119 Pt 4, 1239-1247.

Bonte, M., and Blomert, L,. (2004) . "Developmental changes in ERP correlates of spoken word recognition during early school years: a phonological priming study," Clin Neurophysiol 115, 409-423.

Brodmann, K. (1909) . Vergleichende Lokalisationslehre der Grosshirnrinde. Barth Verlag, Leipzig.

Campain, R., and Minckler, J. (1976) . "A note on the gross configurations of the human auditory cortex," Brain Lang 3, 318-323. 
Cormen, T.H., Leiserson, C.E., Rivest, R.L., Stein, C. (2001) Introduction to Algorithms, 2nd ed. ed. MIT Press and McGraw-Hill, Cambridge, Massachusetts.

Dehaene-Lambertz, G., Dehaene, S., and Hertz-Pannier, L. (2002) . "Functional neuroimaging of speech perception in infants," Science 298, 20132015 .

Dorsaint-Pierre, R., Penhune, V. B., Watkins, K. E., Neelin, P., Lerch, J. P., Bouffard, M., and Zatorre, R. J. (2006) . "Asymmetries of the planum temporale and Heschl's gyrus: relationship to language lateralization," Brain $129,1164-1176$.

Dubois, J., Benders, M., Lazeyras, F., Borradori-Tolsa, C., Leuchter, R. H., Mangin, J. F., and Huppi, P. S. (2010) . "Structural asymmetries of perisylvian regions in the preterm newborn," Neuroimage 52, 32-42.

Frost, M. A., and Goebel, R. (2012) . "Measuring structural-functional correspondence: Spatial variability of specialised brain regions after macroanatomical alignment," Neuroimage 59, 1369-1381.

Geschwind, N., and Levitsky, W. (1968) . "Human brain: left-right asymmetries in temporal speech region," Science 161, 186-187.

Glasel, H., Leroy, F., Dubois, J., Hertz-Pannier, L., Mangin, J. F., and Dehaene-Lambertz, G. (2011) . "A robust cerebral asymmetry in the infant brain: the rightward superior temporal sulcus," Neuroimage 58, 716-723.

Goebel, R., Esposito, F., and Formisano, E. (2006) . "Analysis of functional image analysis contest FIAC data with brainvoyager QX: From singlesubject to cortically aligned group general linear model analysis and selforganizing group independent component analysis," Hum Brain Mapp 27, $392-401$. 
Golestani, N., Molko, N., Dehaene, S., LeBihan, D., and Pallier, C. (2007) . "Brain structure predicts the learning of foreign speech sounds," Cereb Cortex $17,575-582$.

Grossmann, T., Oberecker, R., Koch, S. P., and Friederici, A. D. (2010) . "The developmental origins of voice processing in the human brain," Neuron $65,852-858$.

Hickok, G., and Poeppel, D. (2007) . "The cortical organization of speech processing," Nat Rev Neurosci 8, 393-402.

Hill, J., Dierker, D., Neil, J., Inder, T., Knutsen, A., Harwell, J., Coalson, T., and Van Essen, D. (2010) . "A surface-based analysis of hemispheric asymmetries and folding of cerebral cortex in term-born human infants, " J Neurosci 30, 2268-2276.

Im, K., Jo, H. J., Mangin, J. F., Evans, A. C., Kim, S. I., and Lee, J. M. (2010) . "Spatial distribution of deep sulcal landmarks and hemispherical asymmetry on the cortical surface," Cereb Cortex 20, 602-611.

Kim, J. J., Crespo-Facorro, B., Andreasen, N. C., O'Leary, D. S., Zhang, B., Harris, G., and Magnotta, V. A. (2000) . "An MRI-based parcellation method for the temporal lobe," Neuroimage 11, 271-288.

Klein, M. E., and Zatorre, R. J. (2011) . "A role for the right superior temporal sulcus in categorical perception of musical chords," Neuropsychologia 49, 878-887.

Kriegeskorte, N., and Goebel, R. (2001) . "An efficient algorithm for topologically correct segmentation of the cortical sheet in anatomical mr volumes," Neuroimage 14, 329-346.

Leonard, C. M., Puranik, C., Kuldau, J. M., and Lombardino, L. J. (1998) . "Normal variation in the frequency and location of human auditory cortex landmarks. Heschl's gyrus: where is it?," Cereb Cortex 8, 397-406. 
Ochiai, T., Grimault, S., Scavarda, D., Roch, G., Hori, T., Riviere, D., Mangin, J. F., and Regis, J. (2004) . "Sulcal pattern and morphology of the superior temporal sulcus," Neuroimage 22, 706-719.

Pantazis, D., Joshi, A., Jiang, J., Shattuck, D. W., Bernstein, L. E., Damasio, H., and Leahy, R. M. (2010) . "Comparison of landmark-based and automatic methods for cortical surface registration," Neuroimage 49, 2479-2493.

Penhune, V. B., Zatorre, R. J., MacDonald, J. D., and Evans, A. C. (1996) . "Interhemispheric anatomical differences in human primary auditory cortex: probabilistic mapping and volume measurement from magnetic resonance scans," Cereb Cortex 6, 661-672.

Rauschecker, J. P., and Scott, S. K. (2009) . "Maps and streams in the auditory cortex: nonhuman primates illuminate human speech processing," Nat Neurosci 12, 718-724.

Rogier, O., Roux, S., Belin, P., Bonnet-Brilhault, F., and Bruneau, N. (2010) . "An electrophysiological correlate of voice processing in 4- to 5-year-old children," Int J Psychophysiol 75, 44-47.

Schneider, P., Sluming, V., Roberts, N., Scherg, M., Goebel, R., Specht, H. J., Dosch, H. G., Bleeck, S., Stippich, C., and Rupp, A. (2005) . "Structural and functional asymmetry of lateral Heschl's gyrus reflects pitch perception preference," Nature Neuroscience 8, 1241-1247.

Sharma, A., Kraus, N., McGee, T. J., and Nicol, T. G. (1997) . "Developmental changes in P1 and $\mathrm{N} 1$ central auditory responses elicited by consonant-vowel syllables," Electroencephalogr Clin Neurophysiol 104, 540-545.

Sutherland, M. E., Zatorre, R. J., Watkins, K. E., Herve, P. Y., Leonard, G., Pike, B. G., Witton, C., and Paus, T. (2012) . "Anatomical correlates of dynamic auditory processing: Relationship to literacy during early adolescence," Neuroimage 60, 1287-1295. 
Talairach, J., Tournoux, P. (1980). Co-planar sterotaxic atlas of the human brain. Thieme, Stuttgart.

Vaessen, A., Blomert, L. (2010). Long-term cognitive dynamics of fluent reading development. J Exp Child Psychol 105, 213-231.

Van Essen, D. C. (2005) . "A Population-Average, Landmark- and Surfacebased (PALS) atlas of human cerebral cortex," Neuroimage 28, 635-662.

Warrier, C., Wong, P., Penhune, V., Zatorre, R., Parrish, T., Abrams, D., and Kraus, N. (2009) . "Relating structure to function: Heschl's gyrus and acoustic processing," J Neurosci 29, 61-69.

Watkins, K. E., Paus, T., Lerch, J. P., Zijdenbos, A., Collins, D. L., Neelin, P., Taylor, J., Worsley, K. J., and Evans, A. C. (2001) . "Structural asymmetries in the human brain: a voxel-based statistical analysis of 142 MRI scans," Cereb Cortex 11, 868-877.

Westbury, C. F., Zatorre, R. J., and Evans, A. C. (1999) . "Quantifying variability in the planum temporale: a probability map," Cereb Cortex 9, 392405 .

Wong, P. C., Warrier, C. M., Penhune, V. B., Roy, A. K., Sadehh, A., Parrish, T. B., and Zatorre, R. J. (2008) . "Volume of left Heschl's Gyrus and linguistic pitch learning," Cereb Cortex 18, 828-836.

Yoon, U., Fahim, C., Perusse, D., and Evans, A. C. (2010) . "Lateralized genetic and environmental influences on human brain morphology of 8-yearold twins," Neuroimage 53, 1117-1125.

Zatorre, R. J., Belin, P., and Penhune, V. B. (2002) . "Structure and function of auditory cortex: music and speech," Trends Cogn Sci 6, 37-46. 
SUMMARY 


\section{Summary}

This thesis discusses a body of work which is concentrated around the central theme of improving brain mapping by greater consideration of the anatomy of the brain, while examining the relationship between the human brain's structure and function. As a relatively new science, neuroimaging methods are being developed at a staggering rate. Improvements to fMRI scanner hardware with improved coils allowing for a better measured signal, as well as advancements in sequence programming and the increasing use of high-field scanners, have improved the quality of the measured brain images. Vast advancements have also been made in the analysis of the recorded signals. Advances on the analysis front have focused on extracting different types of information from the brain signals measured with fMRI. Multivariate analysis in particular has allowed researchers to ask questions which simply were not feasible only a number of years ago. Despite these advances many neuroimaging studies fail to address the issues associated with anatomical variability between subjects. As advancements are made and we measure the brain on an ever finer scale, new methods will need to be developed and used to make use of all the detail gained through the use of new hardware and analysis tools. This thesis attempts to address this issue and contains 4 empirical studies where new tools are employed to create accurate maps of the functioning human brain.

Chapter 1 commences with a brief history of map making and how it is only through accurate observation that maps reach their desired state of being a true depiction of a geographic entity. Just as modern map making is able to achieve an incredible degree of precision thanks to technological advances such as GPS and computer databases, brain imaging has advanced in recent years largely through the advancements of new technologies, first among which is fMRI which has allowed high-resolution imaging of activated brain regions in vivo. It is primarily this machine that has allowed for a new era of brain mapping. The first chapter also gives a brief background on brain normalisation and alignment techniques, pointing out the difficulties faced when trying to compare structural and functional brain images across subjects. The most common method employed to enable these comparisons and report the locus of activations is to register individual brains to one of two standard coordinate systems, either the Talairach or Montreal neurological institute (MNI) space. While the widespread use of these systems has proved to be 
useful for the creation of databases as well as giving the opportunity for effective meta-analysis studies, these volumetric spaces are sub-optimal when it comes to group fMRI studies. Advanced techniques that account for intersubject anatomical variability provide a better platform on which to carry out fMRI group studies.

Chapter 2 contains the first empirical study of the thesis and discusses the investigation of the relationship between structure and function through the creation of probabilistic maps. This chapter shows that, having accounted for macro-anatomical variability between subjects through an advanced, surface based alignment technique, there is more overlap across subjects' functional brain areas in comparison to probabilistic maps created from data in Talariach normalised volume space. This gain in overlap is not uniform across the whole cortex however and certain areas gain more. One area which shows a large structural-functional correspondence is the frontal eye fields (FEF), a functional area active during saccadic eye movements. The FEF is consistently located at the junction of the pre-central sulcus and the superior frontal sulcus. The fusiform face area, active during the viewing of faces, on the other hand is not as strongly bound to macro-anatomy as its location varies slightly along an anterior-posterior axis on the fusiform gyrus. In general we observe that areas which gain alot in terms of overlap have a strong structural-functional correspondence. We suppose that the residual spatial variability between subjects, after macro-anatomical variability is minimised, is likely due to micro-anatomical differences which are unobservable in vivo.

Having investigated the effect of anatomical alignment on the spatial correspondence of discrete functional areas in the chapter 2, chapter 3 addresses a similar question, however widespread functional networks are investigated instead. To this end 7 resting state networks were examined and the intersubject spatial correlation was computed to investigate to degree to which functional networks correspond with macro anatomical landmarks. Resting state networks are a collection of separate and distinct connected brain regions which exhibit positive activation levels in the brain of a subject who is at rest, not doing any particular task. By using the data driven analysis method, independent component analysis (ICA), we were able to extract resting state networks from a 6 minute scan where subjects were asked to lie in the scanner and think of nothing in particular. In order to compare the same networks across subjects the self organising group ICA algorithm was used which automatically matches and clusters group maps based on spatial 
similarity. We observed that the inter-subject spatial correlation of these networks was higher after cortex based alignment (CBA) indicating that, just as with discrete functional areas, resting state networks correspond with macro-anatomical landmarks. These first two empirical studies demonstrate the importance of recognising the fact that each individual possesses a unique folding pattern of sulci and gyri.

Upon realising that not every functional brain area is brought together through the alignment of cortical curvature, as CBA does, we investigated whether there are any further steps that could be taken to create a group average brain, i.e. well aligned both in terms of structure and function. To this end we developed a new alignment technique called functionally driven CBA (fCBA). Chapter 4 starts with a description of the current approach used to circumvent the problem of variability in the spatial location of functional areas, that is to carry out a separate localising scan in order to delineate a functional region of interest (ROI) in which further analysis is carried out. In this way two analyses are required, one which zooms in on the time courses of these ROIs, and another "whole brain" analysis. In this chapter we propose that, by using fCBA and creating a group brain which has been created using a procedure integrating both anatomical and functional data from each subject, only one analysis is necessary. By using fCBA one has the improved specificity of a ROI analysis, having aligned functional areas, however is not limited to analysing this smaller patch of cortex.

The thesis finishes with an application of the methods developed in the preceding chapters. to the auditory system. Here we traced the development, of both structure and function from childhood, through adolescence and into adulthood. Chapter 5 focuses on examining spatial variability in both functional regions of the auditory system, localised using tone and voice stimuli, as well as the anatomical regions, by manually labelling the major gyri, sulci and surfaces of the human auditory system. We observed a difference in the inter-subject variability across development. Interestingly the variability of anatomical regions, as well as functional regions, increases during development, in the right, but not the left hemisphere. It was very fitting to apply these methods to the auditory system due to the large degree of variability known to exist, especially in the anatomy of this area. The results suggest that one's interaction with the sound of the world around them literally shapes the brain during the course of development. 
SAMENVATTING 


\section{Samenvatting}

Dit proefschrift beschrijft een serie experimenten met hetzelfde doel: het beter in kaart kunnen brengen van de relatie tussen functie en anatomie in de hersenen door meer aandacht te schenken aan de anatomie van het individuele brein. Het hersenonderzoek is nog altijd nieuw en methoden om hersenactiviteit te meten en kwantificeren, het zogenaamde 'neuroimaging', worden razendsnel ontwikkeld. De (f)MRI scanners zelf worden nog altijd verbeterd; nieuwe 'spoelen' leiden tot een beter signaal, nieuwe scanalgoritmen bieden extra mogelijkheden, evenals de groeiende sterkte van het magnetisch veld ('high-field' scanners). Maar ook in de analyse methoden en software zit sterke vooruitgang.

Nieuwe manieren om naar de scandata te kijken bieden de mogelijkheid om verschillende soorten informatie te krijgen over wat er in de hersenen gebeurt. In het bijzonder de multivariate analyse heeft het mogelijk gemaakt om onderzoeksvragen op te stellen die luttele jaren geleden onmogelijk leken. Echter, ondanks deze vooruitgang is er tot nog toe weinig aandacht geweest voor de variabiliteit tussen de anatomie van het brein tussen proefpersonen, hoewel dit wel van invloed is op het neuroimaging onderzoek.

Juist omdat de snelle ontwikkeling van scanners en analysemethoden zoveel meer detail en informatie biedt, wordt het steeds belangrijker om dergelijke factoren niet te negeren. Dit proefschrift confronteert daarom het probleem en evalueert de invloed van subtiele individuele verschillen in neuroanatomie op het resultaat van standaard hersenonderzoek. In het proefschrift staan vier empirische studies beschreven waarin nieuwe methoden worden gebruikt om accuraat het functioneren van het menselijke brein in kaart te brengen.

Hoofdstuk 1 begint met een korte geschiedenis van cartography: het in kaart brengen van gebieden en processen. Hier wordt beschreven hoe enkel nauwkeurige observatie ons in staat stelt kaarten te ontwerpen die een accurate weergave van de werkelijkheid zijn. Net zoals technologische innovaties als GPS en computer databases een verbazingwekkende mate van nauwkeurigheid mogelijk maken in moderne wereldkaarten, waren het technologische innovaties in de laatste jaren binnen het hersenonderzoek die het mogelijk maakten om het levende brein ('in vivo') in toenemend detail te onderzoe- 
ken. Met name de MRI scanner, en functionele MRI of fMRI, hebben een nieuw tijdperk van 'brain mapping' ingeleid: het nauwkeurig in kaart brengen van functies in het brein. Het eerste hoofdstuk geeft verder een korte achtergrond over normalisatie en uitlijningsmethoden, met een nadruk op de moeilijkheden bij het direct relateren van functie (activiteit) en structuur (anatomie) in een groep proefpersonen.

De meest gebruikte methode om dit soort relaties te leggen in meerdere proefpersonen is om de individuele breinen (en gerelateerde functionele data) enigszins te vervormen, tot ze passen in één van twee standaard coördinaat-systemen: de Talairach of de Montreal Neurological Institute (MNI) space. Hoewel het gebruik van deze standaard referentie systemen nuttig is geweest voor het opzetten van databases en het mogelijk maken van metaanalyses, is deze standaard methode eigenlijk suboptimaal voor het uitvoeren van groeps-studies met fMRI. In deze context is het waarschijnlijk beter om geavanceerdere technieken te gebruiken die rekening houden met de individuele verschillen in neuroanatomie.

Hoofdstuk 2 beschrijft het eerste experiment in dit proefschift. Dit experiment onderzoekt de relatie tussen functie en structuur door het maken van 'probabilistic maps' ('kans-kaarten'). In dit hoofdstuk laten we zien dat het rekening houden met variabiliteit in anatomie tussen proefpersonen door een geavanceerde 'hersenoppervlakte-gebaseerde uitlijn techniek' leidt tot een grotere overlap van functionele hersengebieden tussen proefpersonen in vergelijking met de overlap die volgt uit standaard normalisatie methoden in Talairach space. Deze toename in overlap is echter niet gelijk verdeeld over hersenschors: sommige functionele gebieden laten meer toename in overlap zien en zijn dus gevoeliger voor de gekozen normalisatie methode. Eén gebied dat een grote structurele-functionele overeenkomst laat zien is de FEF (frontal eye field), een gebied dat actief is tijdens het maken van oogbewegingen. De FEF is consistent bij proefpersonen gelocaliseerd in de kruising tussen de pre-centrale sulcus en de superieure frontale sulcus. Een voorbeeld van een gebied dat niet zo consistent gelinkt kan worden aan een anatomische locatie is de fusiform face area (FFA); de anatomische locatie van dit functionele gebied varieert licht in de anterieure-posterieure richting op de fusiforme gyrus. Functionele gebieden die veel toenemen in overlap over proefpersonen na de geavanceerde uitlijnmethode hebben dus een sterke functionele-structuur relatie. Dit is belangrijk om te weten voor neuroimaging onderzoekers. De overgebleven variabiliteit in functionele localisatie, 
na het corrigeren voor macro-anatomie, is waarschijnlijk te wijten aan micro-anatomische verschillen die niet in vivo te meten zijn.

Nadat we in hoofdstuk 2 hebben gekeken naar het effect van anatomische uitlijning op de spatiele overlap van enkele functionele gebieden, kijken we in hoofdstuk 3 naar een gelijksoortig effect op hele functionele netwerken in het brein. Hiervoor onderzochten we 7 'resting-state' netwerken; netwerken in het brein die samenwerken op momenten dat een proefpersoon in rust ligt zonder taak. De inter-individuele spatiele correlatie werd berekend om te evalueren in hoeverre functionele netwerken gelinkt zijn aan macroanatomische ijkpunten. Door het gebruik van ICA (independent component analysis) konden we deze netwerken extraheren uit de scandata van 6 minuten, waarin proefpersonen in de MRI scanner lagen en gevraagd waren om te ontspannen zonder aan iets bijzonders te denken. Het zelf-organiserende ICA algoritme pasten we toe om dezelfde networken te kunnen vergelijken tussen proefpersonen. Dit algoritme vindt automatisch de netwerken die op elkaar lijken in ruimtelijke dimensies. We vonden dat de inter-individuele spatiele correlatie tussen deze netwerken toenam na de methode van cortexbased alignment (CBA) - ofwel het uitlijnen van de individuele anatomische patronen in de hersenschors. Dit laat zien dat, evenals bij de enkele functionele gebieden, resting-state netwerken in het brein gebonden zijn aan macroanatomische ijkpunten. Deze eerste twee empirische studies laten samen zien dat het belangrijk is te begrijpen en erkennen dat individuele proefpersonen unieke patronen hebben in hun hersenanatomie, welke van invloed is op groepsanalyses in neuroimaging.

$\mathrm{Nu}$ duidelijk was dat niet alle functionele gebieden goed in overeenkomst gebracht konden worden tussen proefpersonen door de methode van CBA, keken we of er nog verdere stappen mogelijk waren om de functionele overlap te vergroten ten behoeve van groepsanalyse in neuroimaging. Met dit doel in gedachten ontwierpen we een nieuwe analysemethode genaamd functie-gedreven CBA, ofwel fCBA. Hoofdstuk $\mathbf{4}$ begint met een beschrijving van de huidige wijze waarop omgegaan wordt met de variabiliteit in spatiele locatie van functionele gebieden; namelijk het uitvoeren van een aparte scan om de functionele regio van interesse te bepalen (ROI) in elke proefpersoon, op basis waarvan verdere analyses vervolgens uitgevoerd worden. Deze conventionele benadering omvat twee stappen: één waarbij nader gekeken wordt naar het tijdsverloop van activiteit in de ROIs, en een tweede "wholebrain" analyse waarbij het hele brein in oogschouw genomen wordt. In dit 
hoofdstuk stellen we voor dat het gebruik van fCBA, het creëren van een groepsbrein op basis van zowel anatomische als functionele data, slechts één analysestap vereist. Met fCBA heeft men wel de verbeterde specificeit van een ROI analyse, doordat functionele gebieden uitgelijnd zijn tussen proefpersonen, maar is men niet beperkt tot analyse van enkel dit functionele gebied.

Dit proefschift sluit af met een toepassing van de methoden die ontwikkeld zijn in voorgaande hoofdstukken. In Hoofdstuk 5 onderzoeken we het auditieve systeem en de ontwikkeling ervan van kindertijd tot adolescentie en volwassenheid. Hier evalueren we zowel de spatiele variabiliteit in functionele gebieden in het auditieve systeem, bepaald door presentatie van tonen en stemgeluiden in een fMRI experiment, als de anatomische gebieden, handmatig bepaald door het labelen van de voornaamste gyri en sulci in het menselijk auditief systeem. Dit onderzoek liet zien dat er een verschil in variabiliteit is tussen proefpersonen over de ontwikkeling heen. Interessant hierbij is dat de de variabiliteit van zowel functionele als anatomische gebieden toeneemt met de levensjaren voor de rechter hersenhelft maar niet de linker. Het auditief systeem was een goede keus voor de toepassing van deze methoden, aangezien al bekend was dat er veel variabiliteit is in met name de anatomie van dit systeem. De resultaten suggereren dat menselijke interactie met het geluid in de wereld letterlijk hun brein vormt over de jaren heen. 


\section{ACKNOWLEDGEMENTS}


First and foremost I would like to thank Rainer for giving me the opportunity to do my PhD here in Maastricht. I had the good fortune to be a student in a tutorial group of Rainer's all those years ago and I remember him asking me to consider staying in Maastricht to follow the new Research Master course...well 8 years on and I'm still here. The opportunity to work with a scientist as clever, thoughtful, motivating, interesting, talented, dynamic and supporting has been a real privilege and I'm extremely honoured to have been given the opportunity to continue working with Rainer into the exciting future of Maastricht and in its ever increasing magnetic attraction.

I'd also like to thank my co-promotor Elia. There are few people as straight talking as Elia and it's always a pleasure to have a discussion with someone with as much integrity as he has. We didn't get the opportunity to work together over the course of my $\mathrm{PhD}$ but we did have lunch together many times. These lunches were most enjoyable on Mondays after a weekend where Naples football team had been victorious, but were always enjoyable nonetheless.

Next I'd like to give an American style "Shout out" to my dear friend Nick, a true friend in every sense of the word. He was always there through thick and thin, from studying together for exams during our time as master students, through to letting me DJ for the first time at his $\mathrm{PhD}$ defence party. My life over the last 8 years in Maastricht has been enriched by having such a great "friend" around.

To my office mate who is more than an office mate Tom. A true gentleman who has been a constant source of motivation and at the same time distraction. Whether we are discussing the latest techniques of data analysis, including all the benefits that normalisation of data can offer, or the most recent buying and selling of football players it's been great sharing the $12 \mathrm{~m} 2$ of floor space we call "our office". We started out as office mates but I now count him among my close friends.

To my lovely Italian friends, Giancarlo et Francesco. Always fun to be around. Always quick to offer assistance. I know its terribly cliché but if it wasn't for you guys, my pasta cooking skills wouldn't be what they are today...but more important than that has been the great times we've spent together over the years, Grazie!. Also to Fabrizio, for bringing his Neapoli- 
tan spark and passion, as well as his deep knowledge of RSNs to my thesis. Working with him was a lot of fun and a great learning experience. Et a mio "amico ufficio" siciliano Tommaso, a domani et buono week-endo!

Teresa and Alex. Thanks for letting me practice being a father by often hanging out with you and your family. There aren't many better ways to spend a weekend afternoon having a "Jever" or prosecco in the sun in jardin de ceramique. Thanks also to the whole ceramique gang; Kirsten and Jorma, Anne-Marie and Joris and of course the kiwis, Phillip and Andreas. It's great to feel the trans-tasman camaraderie alive and well in sunny Maastricht. Katja and Ivan also, it's wonderful to hang out with you, whether it's a Fifa night with the air traffic controllers or a dinner party with great food and company, going to your place is always a blast. Viva jamon!

Job, Michele, Federico, Anke, Roberta, Lars, Matteo, Britta, Marin, Aline, Martin, Jan, Anne, Mario (you hero), Thomas, Franzi. Thanks for being great colleagues and laughing at my bad jokes at lunch. I've enjoyed every lunchtime conversation, particularly when the topic turned to cross cultural differences which seemed to pop up at least every week. Oh and my dear friend Kamil, did you know....?

Thanks to Vincent and Joel, who, just a few steps away in the next office were always obliging in their assistance and it was always a pleasure when a short question turned into a long conversation about a wide array of topics. Joel one day I will listen to your music recommendations...one day! Thanks also to Bettina and Amanda, for our short lived, but fruitful mini-group. Also to Milene, with whom it was great to brainstorm and learn from in my introduction into the wonders of developmental and auditory brain research...I think I'll leave both of those fields to the experts for a while.

The whole department would not exist if it wasn't for the amazing work of Christl, Riny and Annemie. Thanks for being there whenever you're needed and always having a solution to any problem thrown your way. I will always remember to use a blue pen!

I'd also like to thank the instrumentation department and IT department. Special thanks to Jordi who has saved my computer from meltdown twice now and had it back up and running within hours! Also to Sven for his assis- 
tance in the MRI lab in the first few years and Lotty in more recent times. Without you guys my data wouldn't be as sound as it is.

To my friends back home who never seem to forget me no matter how long it is between trips home. Even after eight years away in Europe I feel so lucky to be able return home to all those familiar faces and carry on laughing and having a good time as if I never left. Special thanks to Al for always showing me the best of Sydney whenever I am back, to Mike, for putting me up in London and inspiring my by his sense of adventure, and Kieran, for hosting me in Hong Kong and engaging in the most ridiculous, yet most enjoyable conversations possible.

I'd also like to thank my family, mum and dad, Kate, Mad and Ben. If it wasn't for the support and love all of you have shown, whether as a pixilated image thanks to Skype, or in the flesh back home, I wouldn't be the person I am today. I was blessed with an amazing childhood and given fantastic opportunities which I no longer take for granted and am eternally grateful for the role all 5 of you have played in my life thus far. Even though we are separated by a few thousand miles the strength of our family bond is not diminished. Nothing gives me greater pleasure than boarding a plane bound for home, knowing I'm only $24 \mathrm{hrs}$ away from spending time with you guys!

Finally to Aude. I came to Maastricht on an exchange which was meant to last just 6 months however meeting you here 8 years ago changed my life forever. I love your honesty, Swiss practicality, your warmth and your tenderness. I've enjoyed all the adventures we've been on over the last few years and look forward to all our adventures yet to come. You give me the strength to be the person I am and the support to let me follow my dreams. You are an amazing person and I'm so honoured that you are part of my life. Je t'aime de tout mon coeur. 
CURRICULUM VITAE 


\section{Curriculum Vitae}

Martin Frost was born in Sydney, Australia on March $25^{\text {th }} 1982$. He attended St Kevin's Primary School from 1987-1991 before moving to St Aloysius college where he graduated with the higher school certificate in 1999. Martin commenced his tertiary education in 2000 studying for a Bachelor of Arts, with majors in History and Government as well as a Bachelor of Science with a major in Psychology at the University of Sydney. He graduated in 2005. During the final year of his undergraduate studies Martin participated in an exchange program and spent a semester at Maastricht University in The Netherlands. In 2005 he commenced a Research Master degree at Maastricht University. He received the Huygens scholarship from NUFFIC the Dutch organisation for international cooperation in higher education. His master thesis, entitled "The Structural-Functional Correspondence Project" was supervised by Prof. Dr. Rainer Goebel. Martin graduated cum laude in 2007 and was awarded the thesis prize for the Faculty of Psychology. In 2007 he commenced his PhD studies under the guidance of Prof. Goebel at the Department of Cognitive Neuroscience at the Faculty of Psychology and Neuroscience at Maastricht University. He is currently a post-doctoral fellow at the same institute where he continues his research on the relationship between structure and function in the human brain. 
Publications 


\section{Publications}

Frost, M. A., and Goebel, R. (2012) Measuring structural-functional correspondence: Spatial variability of specialised brain regions after macroanatomical alignment, Neuroimage 59, 1369-1381.

Frost, M.A., Esposito, F. \& Goebel, R. (2012) The Structural - Functional Correspondence of Resting State Networks, Human Brain Mapping. In Press.

Frost, M.A. \& Goebel, R. (2012). One or two analyses? An integrated approach for whole-cortex macro-anatomical and ROI-based functional alignment. Submitted.

Bonte, M., Frost, M.A., Rutten, S., Ley, A., Formisano, E. \& Goebel, R. (2012) An investigation into the structural and functional development of human superior temporal cortex. In preparation.

\section{Conference Presentations}

M.A. Frost, R. Goebel (2011) Is manual landmark labelling necessary for optimal macro-anatomical cortex alignment? $17^{\text {th }}$ Meeting of the Organization for Human Brain Mapping. 26-30 June, Quebec City, Canada.

M. A. Frost, R.Goebel (2010) Using Functional Areas as Additional Targets for Multi Subject Brain Normalisation. 16th Meeting of the Organization for Human Brian Mapping, 6-10 June, Barcelona, Spain.

H. Breman, S. Frost, M.A. Okamoto-Barth, J. Peters, J. Zimmermann, C. Hutton, J. Ashburner, N. Weiskopf, and R. Goebel (2010) Quantifying the effect of the susceptibility artifact on volume and surface. 16th Meeting of the Organization for Human Brian Mapping, 6-10 June, Barcelona, Spain.

M.A. Frost, R. Goebel, (2009) Examining Structural - Functional Correspondence by Creating a Functional Probabilistic Atlas. $3^{\text {rd }}$ VogtBrodmann Symposium 4-6 December, Juelich, Germany. 
M.A. Frost, R.Goebel (2009) Creating Functional Probabilistic Maps Using Structurally and Functionally Driven Multi-Subject Alignment. 15th Meeting of the Organization for Human Brain Mapping, 18-23 June, San Francisco, USA.

M.A. Frost, R.Goebel (2008) The Structural-Functional Correspondence Project. 14th Meeting of the Organization for Human Brain Mapping, 15-19 June, Melbourne, Australia.

M.A. Frost, R. Goebel (2007) The Structural-Functional Correspondence Problem. 6th Dutch ENP Conference, 5-8 June, Doorwerth - The Netherlands. 
